\title{
Dimensional reduction of the Standard Model coupled to a new singlet scalar field
}

\author{
Tomáš Brauner, ${ }^{a}$ Tuomas V.I. Tenkanen, ${ }^{b}$ Anders Tranberg, ${ }^{a}$ Aleksi Vuorinen ${ }^{b}$ and \\ David J. Weir ${ }^{a, b}$
}

${ }^{a}$ Faculty of Science and Technology, University of Stavanger, N-4036 Stavanger, Norway

${ }^{b}$ Department of Physics and Helsinki Institute of Physics, P.O. Box 64, FI-00014 University of Helsinki, Finland

E-mail: tomas.brauner@uis.no, tuomas.tenkanen@helsinki.fi, anders.tranberg@uis.no, aleksi.vuorinen@helsinki.fi, david.weir@helsinki.fi

ABSTRACT: We derive an effective dimensionally reduced theory for the Standard Model augmented by a real singlet scalar. We treat the singlet as a superheavy field and integrate it out, leaving an effective theory involving only the Higgs and $\mathrm{SU}(2)_{L} \times \mathrm{U}(1)_{Y}$ gauge fields, identical to the one studied previously for the Standard Model. This opens up the possibility of efficiently computing the order and strength of the electroweak phase transition, numerically and nonperturbatively, in this extension of the Standard Model. Understanding the phase diagram is crucial for models of electroweak baryogenesis and for studying the production of gravitational waves at thermal phase transitions.

Keywords: Beyond Standard Model, Effective field theories, Thermal Field Theory

ARXIV EPRINT: 1609.06230 


\section{Contents}

1 Introduction $\quad 2$

1.1 Background 2

1.2 Dimensional reduction framework 4

2 Standard Model with singlet scalar in Euclidean space 5

2.1 Full four-dimensional theory 6

2.1.1 Renormalization 8

2.1.2 Scaling of parameters 8

$\begin{array}{lll}2.2 & \text { Effective three-dimensional theories } & 9\end{array}$

2.2.1 The superheavy $\sigma$ case 9

$\begin{array}{ll}2.2 .2 \text { The heavy } \sigma \text { case } & 10\end{array}$

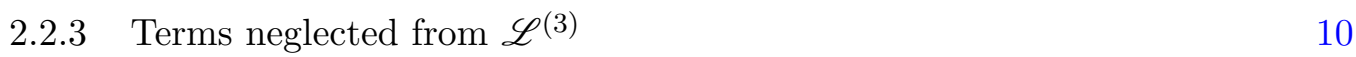

3 Dimensional reduction in the superheavy $\sigma$ case $\quad 11$

$\begin{array}{ll}3.1 \text { Correlators for the dimensional reduction } & 12\end{array}$

$\begin{array}{lll}3.1 .1 & \text { Self-energy diagrams } & 12\end{array}$

$\begin{array}{lll}3.1 .2 & \text { Correlators with gauge fields } & 13\end{array}$

$\begin{array}{lll}3.1 .3 & \text { Effective potential for the scalars } & 17\end{array}$

$\begin{array}{lll}\text { 3.1.4 Scalar correlators from the effective potential } & 18\end{array}$

$\begin{array}{lll}3.2 & \text { Counterterms and } \beta \text {-functions } & 20\end{array}$

$\begin{array}{ll}3.3 \text { Matching relations } & 22\end{array}$

3.3.1 Thermal masses and normalization of fields 22

$\begin{array}{lll}3.3 .2 & \text { Coupling constants } & 24\end{array}$

3.3.3 Collected matching relations at one-loop order 27

$\begin{array}{lll}3.4 & \text { Integration over the heavy scale } & 29\end{array}$

$\begin{array}{lll}3.5 & \text { Relations to physical parameters } & 29\end{array}$

4 Discussion $\quad 30$

A Feynman rules in the unbroken phase $\quad \mathbf{3 5}$

$\begin{array}{lll}\text { A.1 Propagators in the Landau gauge } & 35\end{array}$

$\begin{array}{lll}\text { A.2 Interaction vertices } & 35\end{array}$

$\begin{array}{ll}\text { B Integrals for the dimensional reduction step } & 38\end{array}$

$\begin{array}{lll}\text { B.1 Massless bosonic sum-integrals } & 38\end{array}$

$\begin{array}{lll}\text { B.2 } & \text { Massless fermionic sum-integrals } & 39\end{array}$

$\begin{array}{lll}\text { B.3 Massive sum-integrals } & 39\end{array}$ 


\section{Introduction}

\subsection{Background}

Quantitatively understanding the origin of the observed matter-antimatter asymmetry in the present-day universe is one of the major open challenges in cosmology. A widely studied scenario is that of electroweak baryogenesis $[1,2]$ (see refs. [3, 4] for reviews). This assumes that the excess in baryon density was generated during the electroweak phase transition in the early universe, when the Higgs field obtained its nonzero vacuum expectation value (VEV). While all of the main ingredients for generating a baryon asymmetry can be found in the Standard Model (SM) - an electroweak phase transition as well as the breaking of charge conjugation $(\mathrm{C})$, parity $(\mathrm{P}), \mathrm{CP}$ and baryon number symmetries - it unfortunately turns out that purely SM electroweak baryogenesis fails to live up to its promise.

The problem originates on the one hand from the severe suppression of $\mathrm{CP}$ violation at high temperatures [5-12], and perhaps even more importantly from the fact that the electroweak phase transition within the Standard Model is not of first order, but merely of the crossover type. This conclusion was reached in the mid-1990s after extensive efforts to build a dimensionally reduced effective theory to describe the long-distance dynamics of the SM close to the phase transition [13], and to subsequently study it via nonperturbative lattice simulations [14-16]. Later studies also confirmed this result with four-dimensional simulations [17-19].

As a result of these studies, alternative scenarios such as leptogenesis [20, 21] (see refs. [22, 23] for comprehensive reviews) and cold electroweak baryogenesis [24-27] have been suggested to explain the observed baryon asymmetry. What is common to these scenarios is that they involve degrees of freedom beyond the Standard Model, albeit sometimes at much higher energy scales. There are, however, many sound reasons to expect new physics around the $\mathrm{TeV}$ scale, and a plethora of different scenarios have been proposed to describe this new physics. It is clearly reasonable to investigate whether electroweak baryogenesis might be viable within these models.

Given such a model of new physics at the $\mathrm{TeV}$ scale, the only degrees of freedom requiring nonperturbative treatment at high temperatures are known to be the static modes of the bosonic fields. Following the strategy taken in the original SM works [13-15], the task therefore becomes to first derive three-dimensional effective theories for these modes, and subsequently perform lattice studies of these dimensionally reduced theories.

The recent direct observation of gravitational waves [28] further strengthens the interest in investigating high-energy phase transitions in the early universe. The gravitational waves sourced by bubble collisions and the subsequent nonequilibrium dynamics of a first-order 
electroweak-scale phase transition may be within the sensitivity range of the space-based detector eLISA [29-32], due for launch in 2034. Understanding the strength of such a phase transition in extensions of the Standard Model makes the detection or absence of such primordial gravitational waves a valuable source of information about particle physics; information that is complementary to collider experiments (see e.g. ref. [33] for a related discussion). Indeed, eLISA may be able to probe new physics at temperatures above $10 \mathrm{TeV}$, a region beyond the reach of proposed colliders [32].

In this paper, we shall focus on one such model, the singlet-extended Standard Model (SSM) [34-39], which has been studied in various different contexts including even inflationary physics [40, 41]. This has, in its most general form, seven parameters in the scalar sector, of which two are fixed by the experimental values of the Higgs mass and the Higgs VEV. The remaining five-dimensional parameter space is a challenge to scan, which explains why no comprehensive attempt at a nonperturbative study has been made. The common approach has been a semi-analytic daisy-resummed one-loop effective potential treatment [42-51], which allows for a complete sampling of the parameter space and direct comparison with experimental constraints. However, it is known that perturbative treatments tend to over-estimate the strength of the phase transition $[14,15]$. Hence we expect that the region of (strongly) first order phase transitions is smaller than what has so far been identified. In the present work, we derive the dimensionally reduced effective theory for the SSM. This will be used in simulations, to be detailed in a companion paper [52]. Our main result here is a set of explicit matching relations, which allow us to relate a given set of four-dimensional SSM parameters (and temperature) to the (fewer) parameters of the three-dimensional theory.

We shall present our computation in a highly explicit manner, displaying most of the intermediate results and presenting the final results in a such a form that the Standard Model limit is simple to take. There are two reasons for this. First, the original derivation of the dimensionally reduced effective theory of SM, carried out in the seminal paper [13], was presented in a rather compact way, suppressing many calculational details. Second, apart from the SSM, it is naturally very interesting and well-motivated to study baryogenesis in a number of other beyond-SM models, which could be subjected to the same procedure presented here. We hope that by providing more details of the calculations, our work will be useful for a broader audience interested in the derivation or use of dimensionally reduced effective theories either in the SM or in different beyond-SM scenarios.

A note of caution is, however, necessary. In our derivation of the dimensionally reduced effective field theory, we work to one loop order for all the parameters of the effective theory and only perform the matching to physical parameters at tree level. While this does not match the accuracy of the original Standard Model calculation performed in ref. [13], we do not expect this to affect the phenomenological implications of our calculation. Nevertheless we shall revisit this issue in ref. [52].

This paper is organized as follows. In the remainder of this introductory section, we explain the basic principles of dimensional reduction on a very general level. In section 2, we then introduce the SSM, including the forms of its four- and three-dimensional Lagrangians as well as the associated parameters. The actual dimensional reduction of the model is 
performed in section 3. In section 4, we discuss our findings and investigate the extent to which the inclusion of a scalar singlet improves the prospects for a first order phase transition. Many details of the calculations, ranging from Feynman rules to the results for individual graphs, are deferred to the appendices.

\subsection{Dimensional reduction framework}

Dimensional reduction is a generic physical principle governing the properties of quantum field theories at high temperatures, stating that the low-energy behavior of static Green's functions can be determined through a lower-dimensional effective theory. In short, it follows from the fact that in thermal equilibrium, four-dimensional fields can be reduced to infinite towers of three-dimensional field modes - termed Matsubara modes — by means of a Fourier series expansion in the imaginary time variable $\tau$. The effective masses of the three-dimensional fields become

$$
M_{\text {boson }}^{2}=M_{0}^{2}+(2 n \pi T)^{2}, \quad M_{\text {fermion }}^{2}=M_{0}^{2}+[(2 n+1) \pi T]^{2},
$$

where $M_{0}$ denotes the field mass at zero temperature and $n$ takes integer values. Consequently, at high temperatures, that is for $T \gtrsim M_{0}$ for all fields, all modes except for the bosonic zero modes $(n=0)$ obtain thermal masses at least of order $\pi T$, and thus decouple from physics at length scales parametrically larger than $1 / T$.

Let us now follow the discussion of ref. [13] and specialize to a model whose bosonic sector can be described via a Euclidean Lagrangian density of the generic form

$$
\mathscr{L}=\frac{1}{4} F_{\mu \nu} F_{\mu \nu}+D_{\mu} \phi^{\dagger} D_{\mu} \phi+\mu^{2} \phi^{\dagger} \phi+\lambda\left(\phi^{\dagger} \phi\right)^{2}+g_{Y} \bar{\psi} \phi \psi+\delta \mathscr{L},
$$

where $A_{\mu}$ (appearing inside $F_{\mu \nu}$ and $D_{\mu}$ ) is a gauge field, $\phi$ a complex scalar, $\psi$ a fermion, and $\delta \mathscr{L}$ corresponds to counterterms. We further assume that the Yukawa coupling $g_{Y}$ and the scalar self-coupling $\lambda$ scale as $g_{Y} \sim g$ and $\lambda \sim g^{2}$ in terms of the gauge coupling $g$. Then it can be verified that at one-loop order, interactions contribute to the masses of the zero Matsubara modes of the $\phi, A_{0}$ and $A_{r}$ fields ${ }^{1}$ as

$$
M_{\phi}^{2}-M_{0}^{2} \sim g^{2} T^{2}, \quad M_{A_{0}}^{2} \sim g^{2} T^{2}, \quad M_{A_{r}}^{2}=0,
$$

where the last of the relations is consistent with the fact that the dimensionally reduced effective theory possesses three-dimensional gauge invariance.

From the above considerations, we see the emergence of a scale hierarchy in the system. The thermal scale $\pi T$ is canonically dubbed superheavy, while the mass scale of the $A_{0}$ field, $g T$, is referred to as heavy. Finally, the mass of the $\phi$ field depends on the value of the mass parameter $M_{0}$ : should $M_{0}$ be comparable to $\pi T$, the corresponding field mode is treated as superheavy, whereas for $M_{0}$ of order $g T$, it is heavy. An exception may, however, occur near a phase transition, where the $\mathcal{O}\left(g^{2} T^{2}\right)$ one-loop correction to $M_{\phi}^{2}$ exactly cancels the (negative) tree-level $M_{0}^{2}$. In this case, the mass of the $n=0$ mode of the scalar field

\footnotetext{
${ }^{1}$ In order to avoid confusion with the isospin doublet index $i, j, \ldots$, employed for the Higgs field and the SM fermions, we use the letters $r, s, \ldots$ to label spatial vectors.
} 
becomes of order $g^{2} T$ and the field is referred to as light. The $n=0$ component of the spatial gauge fields $A_{r}$, which is protected by gauge invariance, is naturally light as well.

The formal procedure of dimensional reduction consists of successively integrating out the superheavy and heavy energy scales from the system. This implies deriving effective Lagrangians for the relevant field modes, which is most easily done with the following recipe (see e.g. refs. [53, 54]):

1. Determine the relevant light degrees of freedom of the effective theory.

2. Write down the most general local Lagrangian consistent with the symmetries of the theory, including three-dimensional gauge invariance.

3. Order the operators in the Lagrangian in terms of their dimensions and discard terms beyond a given order.

The essence of dimensional reduction is that the three-dimensional effective theory obtained with the above procedure is capable of reproducing the long-distance - length scales $1 /(g T)$ and above - Green's functions of the full four-dimensional theory. This can be done to arbitrary accuracy, provided that operators of high enough dimension are included in the corresponding Lagrangian density. In practice, this implies matching various Green's functions for the two theories, and deriving from them expressions for the parameters of the effective theory.

Let us now specialize to the case of a high-temperature phase transition, and assume that the thermal correction to the mass of the $n=0$ scalar field mode exactly cancels its negative zero-temperature mass parameter, so that the field becomes light. In this case, dimensional reduction proceeds in two successive stages. In the first step, we integrate out only the superheavy modes, leaving behind a three-dimensional superrenormalizable effective theory for the spatial gauge field $A_{r}$, the massive temporal gauge field $A_{0}$, and the scalar $\phi$. This theory is capable of describing physics at length scales $1 /(g T)$, but still contains two distinct scales: the $\mathcal{O}(g T)$ mass of $A_{0}$ and the $\mathcal{O}\left(g^{2} T\right)$ mass of $\phi$. The former can then also be integrated out, leaving a theory for the light modes only, i.e. the fields $A_{r}$ and $\phi$. The construction of the Lagrangians and the matching calculations needed for the determination of the corresponding parameters are discussed at length in ref. [13].

For the remainder of this paper, we take the basic principles of dimensional reduction as given, referring the interested reader to refs. [13, 53, 54]. These principles will be applied to the study of the SSM, which is introduced in the next section. There, we shall also write down the explicit forms of the effective Lagrangians corresponding to two different scenarios where the new singlet scalar is treated as superheavy and heavy, respectively, even though we shall only carry out the dimensional reduction in the superheavy case. The matching calculations are then presented in the following section, which is dedicated to the case where the extra singlet is superheavy.

\section{Standard Model with singlet scalar in Euclidean space}

In this section, we review the Standard Model coupled to a singlet scalar field. In addition, we present the form of the three-dimensional effective Lagrangians for two scenarios, 
in which the singlet is treated as superheavy and heavy, respectively. Throughout the discussion, we shall work in a Euclidean spacetime of $D=d+1=4-2 \epsilon$ dimensions.

\section{$2.1 \quad$ Full four-dimensional theory}

The classical Euclidean Lagrangian of our four-dimensional theory reads

$$
\mathscr{L}=\mathscr{L}_{\text {gauge }}+\mathscr{L}_{\text {ghost }}+\mathscr{L}_{\text {fermion }}+\mathscr{L}_{\text {scalar }}+\mathscr{L}_{\text {Yukawa }}+\delta \mathscr{L}
$$

where the gauge field, ghost, fermion, scalar and Yukawa sector Lagrangians are defined as follows (the counterterm part $\delta \mathscr{L}$ will be discussed later):

$$
\begin{aligned}
\mathscr{L}_{\text {gauge }}= & \frac{1}{4} G_{\mu \nu}^{a} G_{\mu \nu}^{a}+\frac{1}{4} F_{\mu \nu} F_{\mu \nu}+\frac{1}{4} H_{\mu \nu}^{\alpha} H_{\mu \nu}^{\alpha}, \\
\mathscr{L}_{\text {ghost }}= & \partial_{\mu} \bar{\eta}^{a} D_{\mu} \eta^{a}+\partial_{\mu} \bar{\xi} \partial_{\mu} \xi+\partial_{\mu} \bar{\zeta}^{\alpha} D_{\mu} \zeta^{\alpha}, \\
\mathscr{L}_{\text {fermion }}= & \sum_{A}\left(\bar{\ell}_{A} \not D \ell_{A}+\bar{e}_{A} \not D e_{A}+\bar{q}_{A} \not D_{q_{A}}+\bar{u}_{A} \not D u_{A}+\bar{d}_{A} \not D d_{A}\right), \\
\mathscr{L}_{\text {scalar }}= & D_{\mu} \phi^{\dagger} D_{\mu} \phi-\mu_{h}^{2} \phi^{\dagger} \phi+\lambda_{h}\left(\phi^{\dagger} \phi\right)^{2}+\frac{1}{2}\left(\partial_{\mu} \sigma\right)^{2}+\frac{1}{2} \mu_{\sigma}^{2} \sigma^{2} \\
& +\mu_{1} \sigma+\frac{1}{3} \mu_{3} \sigma^{3}+\frac{1}{4} \lambda_{\sigma} \sigma^{4}+\frac{1}{2} \mu_{m} \sigma \phi^{\dagger} \phi+\frac{1}{2} \lambda_{m} \sigma^{2} \phi^{\dagger} \phi, \\
\mathscr{L}_{\text {Yukawa }}= & \sum_{A, B}\left[h_{A B}^{(e)} \bar{\ell}_{A} e_{B} \phi+h_{A B}^{(d)} \bar{q}_{A} d_{B} \phi+h_{A B}^{(u)} \bar{q}_{A} u_{B} \tilde{\phi}\right]+\text { h.c. }
\end{aligned}
$$

We shall work in the Landau gauge. The theory includes the following fields:

- The $\mathrm{SU}(2)_{L}, \mathrm{U}(1)_{Y}$ and $\mathrm{SU}(3)_{c}$ gauge fields $A_{\mu}^{a}, B_{\mu}$, and $C_{\mu}^{\alpha}$ appearing inside the field strength tensors $G_{\mu \nu}^{a}, F_{\mu \nu}$ and $H_{\mu \nu}^{\alpha}$. The associated gauge couplings are $g, g^{\prime}$, and $g_{s}$, and the corresponding ghost fields $\eta^{a}, \xi$, and $\zeta^{\alpha}$.

- The left-handed doublet and right-handed singlet lepton fields with a flavor index, $\ell_{A}$ and $e_{A}$, as well as the left-handed doublet quark fields $q_{A}$ and right-handed singlet up- and down-type quark fields $u_{A}$ and $d_{A}$.

- The Higgs field $\phi^{i}$, with the charge-conjugated Higgs doublet $\tilde{\phi} \equiv \mathrm{i} \tau_{2} \phi^{*}$, where $\tau_{2}$ is the second Pauli matrix.

- The extra real singlet scalar field $\sigma$.

The relation $Q=I_{3}+\frac{Y}{2}$ between electric charge $Q$ and isospin $I_{3}$ defines the hypercharge of the fields as follows: $Y_{\ell}=-1, Y_{e}=-2, Y_{q}=\frac{1}{3}, Y_{u}=\frac{4}{3}, Y_{d}=-\frac{2}{3}, Y_{\phi}=1$, $Y_{\sigma}=0$. Finally, we shall for completeness write down explicit expressions for the covariant 
derivatives and field strength tensors. The covariant derivatives read in different cases

$$
\begin{aligned}
D_{\mu} \psi & =\left(\partial_{\mu}-\mathrm{i} g \frac{\vec{\tau}}{2} \cdot \vec{A}_{\mu}-\mathrm{i} g_{s} \frac{\vec{\lambda}}{2} \cdot \vec{C}_{\mu}-\mathrm{i} g^{\prime} \frac{Y}{2} B_{\mu}\right) \psi & & \left(\text { for } q_{A}\right), \\
D_{\mu} \psi & =\left(\partial_{\mu}-\mathrm{i} g \frac{\vec{\tau}}{2} \cdot \vec{A}_{\mu}-\mathrm{i} g^{\prime} \frac{Y}{2} B_{\mu}\right) \psi & & \left(\text { for } \ell_{A}, \phi\right), \\
D_{\mu} \psi & =\left(\partial_{\mu}-\mathrm{i} g_{s} \frac{\vec{\lambda}}{2} \cdot \vec{C}_{\mu}-\mathrm{i} g^{\prime} \frac{Y}{2} B_{\mu}\right) \psi & & \left(\text { for } u_{A}, d_{A}\right), \\
D_{\mu} \psi & =\left(\partial_{\mu}-\mathrm{i} g^{\prime} \frac{Y}{2} B_{\mu}\right) \psi & & \left(\text { for } e_{A}, \sigma\right),
\end{aligned}
$$

where $\vec{\tau}$ and $\vec{\lambda}$ denotes the vector of Pauli and Gell-Mann matrices, respectively. Finally, the field strength tensors take the forms

$$
\begin{aligned}
G_{\mu \nu}^{a} & =\partial_{\mu} A_{\nu}^{a}-\partial_{\nu} A_{\mu}^{a}+g \epsilon_{b c}^{a} A_{\mu}^{b} A_{\nu}^{c}, \\
F_{\mu \nu} & =\partial_{\mu} B_{\nu}-\partial_{\nu} B_{\mu}, \\
H_{\mu \nu}^{\alpha} & =\partial_{\mu} C_{\nu}^{\alpha}-\partial_{\nu} C_{\mu}^{\alpha}+g_{s} f_{\beta \gamma}^{\alpha} C_{\mu}^{\beta} C_{\nu}^{\gamma} .
\end{aligned}
$$

In the Yukawa part $\mathscr{L}_{\text {Yukawa }}, h^{(e)}, h^{(d)}$ and $h^{(u)}$ stand for the flavor-mixing matrices, while h.c. represents hermitian conjugate. In the final stages of our calculation, we shall use an approximation where only the top quark Yukawa coupling $g_{Y}$ is nonzero. The Yukawa sector then simplifies to

$$
\mathscr{L}_{\text {Yukawa }}=g_{Y}\left(\bar{q}_{t} \tilde{\phi} t+\bar{t} \tilde{\phi}^{\dagger} q_{t}\right) \quad \text { (if top-quark only) }
$$

For the sake of convenience, the Feynman rules in the unbroken phase of this theory are listed in appendix A.

Since $\sigma$ is a real singlet, we can choose ${ }^{2}$ the zero-temperature VEV, around which we perturb, to be at $\sigma=0$. This shift amounts to a redefinition of the parameters of the potential, and since $\sigma=0$ is defined to be a minimum, we have that $\mu_{\sigma}^{2} \geq 0$. Our choice also imposes a relation between $\mu_{1}$ and $\mu_{m}$ in the vacuum where the Higgs field has a VEV, given by $\left\langle\phi^{\dagger} \phi\right\rangle=v^{2} / 2$,

$$
\mu_{1}=-\frac{\mu_{m} v^{2}}{4}
$$

To start with, however, we will not impose this constraint, treating $\mu_{1}$ and $\mu_{m}$ as independent parameters. Keeping the parameter $\mu_{1}$ explicit will allow us to see in section 3.3.3 that the matching relations for the three-dimensional parameters are independent of the renormalization scale of the four-dimensional theory; including the running of $\mu_{1}$ is essential to ensure this property. Later on, in section 3.5, we shall impose the condition (2.15) when we relate the $\overline{\mathrm{MS}}$ scheme parameters to physical observables in the vacuum. We will assume throughout that $\mu_{\sigma}^{2}>0$. As argued above, this represents no loss of generality.

\footnotetext{
${ }^{2} \mathrm{~A}$ similar shift is not permitted for the Higgs field because of gauge invariance.
} 


\subsubsection{Renormalization}

All fields and couplings appearing in the above Lagrangian are the renormalized ones, while the counterterms, given explicitly in section 3.2, are included in $\delta \mathscr{L}$. We use the following conventions for the relations between the renormalized fields and couplings and their bare counterparts, denoted by the subscript $(b)$ :

$$
\begin{aligned}
\vec{A}_{\mu(b)} & \equiv Z_{A}^{1 / 2} \vec{A}_{\mu}=\left(1+\delta Z_{A}\right)^{1 / 2} \vec{A}_{\mu}, \\
B_{\mu(b)} & \equiv Z_{B}^{1 / 2} B_{\mu}=\left(1+\delta Z_{B}\right)^{1 / 2} B_{\mu}, \\
\phi_{(b)} & \equiv Z_{\phi}^{1 / 2} \phi=\left(1+\delta Z_{\phi}\right)^{1 / 2} \phi \\
\sigma_{(b)} & \equiv Z_{\sigma}^{1 / 2} \sigma=\left(1+\delta Z_{\sigma}\right)^{1 / 2} \sigma,
\end{aligned}
$$

for the fields, and

$$
\begin{aligned}
g_{(b)} & \equiv g+\delta g, \quad g_{(b)}^{\prime} \equiv g^{\prime}+\delta g^{\prime}, & & g_{Y(b)} \equiv g_{Y}+\delta g_{Y}, \\
\mu_{h(b)}^{2} & \equiv Z_{\phi}^{-1}\left(\mu_{h}^{2}+\delta \mu_{h}^{2}\right), & & \lambda_{h(b)} \equiv Z_{\phi}^{-2}\left(\lambda_{h}+\delta \lambda_{h}\right), \\
\mu_{1(b)} & \equiv Z_{\sigma}^{-1 / 2}\left(\mu_{1}+\delta \mu_{1}\right), & \mu_{\sigma(b)}^{2} & \equiv Z_{\sigma}^{-1}\left(\mu_{\sigma}^{2}+\delta \mu_{\sigma}^{2}\right), \\
\mu_{3(b)} & \equiv Z_{\sigma}^{-3 / 2}\left(\mu_{3}+\delta \mu_{3}\right), & \mu_{m(b)} & \equiv Z_{\phi}^{-1} Z_{\sigma}^{-1 / 2}\left(\mu_{m}+\delta \mu_{m}\right), \\
\lambda_{\sigma(b)} & \equiv Z_{\sigma}^{-2}\left(\lambda_{\sigma}+\delta \lambda_{\sigma}\right), & \lambda_{m(b)} & \equiv Z_{\phi}^{-1} Z_{\sigma}^{-1}\left(\lambda_{m}+\delta \lambda_{m}\right),
\end{aligned}
$$

for the couplings. It is worth pointing out that at the one-loop level at which we work, the singlet scalar does not receive any wavefunction renormalization, that is, $Z_{\sigma}=1$.

\subsubsection{Scaling of parameters}

We assume that the parameters of the theory obey the following parametric scaling relations in terms of the $\mathrm{SU}(2)_{L}$ coupling $g$ :

- $g^{\prime}, g_{s}, g_{Y} \sim g$,

- $\lambda_{h}, \lambda_{m}, \lambda_{\sigma} \sim g^{2}$,

- $\mu_{h}, \mu_{3} \sim g T$,

- $\mu_{1} \sim g T^{3}$,

- $\mu_{m} \sim g^{n} T$, and $\mu_{\sigma} \sim g^{m} T$,

where we keep some freedom in the choice of the scaling power for the mass and cubic interaction of the singlet scalar. To find a suitable choice for $m$ and $n$, consider schematically the tree-level contribution to the Higgs four-point function originating from a $\sigma$ exchange at vanishing external momenta,

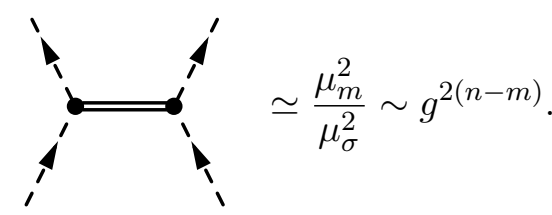


We require this contribution to be at least of order $g^{2}$, so that it does not exceed the value of the Higgs self-coupling $\lambda_{h}$. We therefore have two interesting and very distinctive options: superheavy $\sigma$ (corresponding to $m=0$ ) combined with $n=1$, and heavy $\sigma$ (corresponding to $m=1$ ), combined with $n=2$.

In the first case, even the zero mode of $\sigma$ is superheavy and will therefore be integrated out, together with the non-vanishing Matsubara modes. The three-dimensional effective theory is then, up to operators of order six and higher in the fields, the same as in the Standard Model. However, the dimensional reduction step contains new technical aspects compared to the Standard Model case considered in ref. [13], as one cannot expand the superheavy $\sigma$ mass term in the denominator of sum-integrals, but has to consider massive sum-integrals instead, cf. section B. Furthermore, in addition to the oneparticle-irreducible (1PI) diagrams usually sufficient for matching of the four-dimensional and three-dimensional theories, one needs to include graphs which are one- $\sigma$-reducible.

When $\sigma$ is itself heavy, it remains in the dimensionally reduced theory for the heavy scale. Sum-integrals with $\sigma$ propagators can then be expanded in the mass parameter, which generates higher-order corrections analogous to those stemming from the Higgs mass parameter. Moreover, in this case the contributions originating from the coupling $\mu_{m}$ are highly suppressed.

We emphasize that our scaling relations above differ from those of ref. [13]; we do not assume $g^{\prime}$ to be parametrically smaller than $g$. As a result, we have to retain the $\mathrm{U}(1)_{Y}$ gauge field, treating it on the same footing as the $\mathrm{SU}(2)_{L}$ gauge field.

\section{$2.2 \quad$ Effective three-dimensional theories}

Unless explicitly stated otherwise, we choose to denote the fields of the effective theories with the same symbols as those of the four-dimensional theory. However, the effective theory gauge couplings are denoted by $g_{3}, g_{3}^{\prime}$ and $g_{s, 3}$. The classical Lagrangian density of the effective theory (again in the Landau gauge) then has the schematic form

$$
\mathscr{L}^{(3)}=\mathscr{L}_{\text {gauge }}^{(3)}+\mathscr{L}_{\text {ghost }}^{(3)}+\mathscr{L}_{\text {scalar }}^{(3)}+\mathscr{L}_{\text {temporal }}^{(3)}+\delta \mathscr{L}^{(3)} .
$$

We include the $\mathrm{SU}(2)_{L}$ and $\mathrm{U}(1)_{Y}$ gauge fields in the gauge sector part

$$
\mathscr{L}_{\text {gauge }}^{(3)}=\frac{1}{4} G_{r s}^{a} G_{r s}^{a}+\frac{1}{4} F_{r s} F_{r s},
$$

where only spatial Lorentz indices are summed over. The explicit forms of $\mathscr{L}_{\text {ghost }}^{(3)}$ and $\delta \mathscr{L}^{(3)}$ are not relevant for the present discussion. The scalar and temporal gauge field sectors are discussed below for our two different cases.

\subsubsection{The superheavy $\sigma$ case}

As explained above, in this case the neutral scalar is completely integrated out in the dimensional reduction step. To the order we are working, the three-dimensional Lagrangian 
therefore coincides with that of SM, with the temporal gauge field part reading

$$
\begin{aligned}
\mathscr{L}_{\text {temporal }}^{(3)}= & \frac{1}{2}\left(D_{r} A_{0}^{a}\right)^{2}+\frac{1}{2} m_{D}^{2} A_{0}^{a} A_{0}^{a}+\frac{1}{2}\left(\partial_{r} B_{0}\right)^{2}+\frac{1}{2} m_{D}^{\prime 2} B_{0}^{2}+\frac{1}{2}\left(D_{r} C_{0}^{\alpha}\right)^{2} \\
& +\frac{1}{2} m_{D}^{\prime \prime 2} C_{0}^{\alpha} C_{0}^{\alpha}+\frac{1}{4} \lambda_{3}\left(A_{0}^{a} A_{0}^{a}\right)^{2}+\frac{1}{4} \lambda_{3}^{\prime} B_{0}^{4}+\frac{1}{4} \lambda_{3}^{\prime \prime} A_{0}^{a} A_{0}^{a} B_{0}^{2}+h_{3} \phi^{\dagger} \phi A_{0}^{a} A_{0}^{a} \\
& +h_{3}^{\prime} \phi^{\dagger} \phi B_{0}^{2}+h_{3}^{\prime \prime} B_{0} \phi^{\dagger} \vec{A}_{0} \cdot \vec{\tau} \phi+\delta_{3} \phi^{\dagger} \phi C_{0}^{\alpha} C_{0}^{\alpha},
\end{aligned}
$$

with the covariant derivatives of the adjoint fields reading $D_{r} A_{0}^{a}=\partial_{r} A_{0}^{a}+g_{3} \epsilon^{a}{ }_{b c} A_{r}^{b} A_{0}^{c}$ and $D_{r} C_{0}^{\alpha}=\partial_{r} C_{0}^{\alpha}+g_{s} f_{\beta \rho}^{\alpha} C_{r}^{\beta} C_{0}^{\rho}$.

Finally, the scalar part of the Lagrangian is

$$
\mathscr{L}_{\text {scalar }}^{(3)}=D_{r} \phi^{\dagger} D_{r} \phi-\mu_{h, 3}^{2} \phi^{\dagger} \phi+\lambda_{h, 3}\left(\phi^{\dagger} \phi\right)^{2} .
$$

In this case, the second step of dimensional reduction from the heavy to the light scale is identical to that of SM, with the heavy temporal gauge fields $A_{0}^{a}, B_{0}$ and $C_{0}^{\alpha}$ integrated out. The results for the parameters of the effective theory for the light scale, denoted by $\bar{g}_{3}, \bar{g}_{3}^{\prime}, \bar{\mu}_{h, 3}, \bar{\lambda}_{h, 3}$, can be taken from ref. [13] (apart from the contribution of temporal gluon fields $C_{0}^{\alpha}$ ), and are therefore only briefly reviewed in section 3.4. In ref. [13], gluons were completely neglected from the three-dimensional theory, expecting the effect of this omission to be subdominant in the final conclusions regarding the order and properties of the electroweak phase transition. We have, however, included the leading order contribution from temporal gluons for completeness.

\subsubsection{The heavy $\sigma$ case}

When the $\sigma$ field is heavy, the static (zero Matsubara) mode of the $\sigma$ field appears in the effective theory for the heavy scale, resulting in additional terms in the Lagrangian. The scalar part $\mathscr{L}_{\text {scalar }}^{(3)}$ now includes the operators

$$
\frac{1}{2}\left(\partial_{r} \sigma\right)^{2}+\mu_{1,3} \sigma+\frac{1}{2} \mu_{\sigma, 3}^{2} \sigma^{2}+\frac{1}{3} \mu_{3,3} \sigma^{3}+\frac{1}{4} \lambda_{\sigma, 3} \sigma^{4}+\frac{1}{2} \mu_{m, 3} \sigma \phi^{\dagger} \phi+\frac{1}{2} \lambda_{m, 3} \sigma^{2} \phi^{\dagger} \phi,
$$

while $\mathscr{L}_{\text {temporal }}^{(3)}$ acquires the new terms

$$
x_{3} \sigma A_{0}^{a} A_{0}^{a}+x_{3}^{\prime} \sigma B_{0}^{2}+y_{3} \sigma^{2} A_{0}^{a} A_{0}^{a}+y_{3}^{\prime} \sigma^{2} B_{0}^{2}+y_{3}^{\prime \prime} \sigma \phi^{\dagger} \vec{A}_{0} \cdot \vec{\tau} \phi .
$$

The derivation of the effective theory for the light scale differs from the SM computation in that one needs to integrate out the zero mode of $\sigma$. Although in principle straightforward, this calculation is left for future work. For the remainder of this paper, we focus exclusively on the superheavy $\sigma$ case, where the singlet scalar is completely integrated out already in the first dimensional reduction step.

\subsubsection{Terms neglected from $\mathscr{L}^{(3)}$}

Before we close this section, we will briefly list and discuss examples of operators that have been discarded from the three-dimensional effective theory for various reasons: 
- The effects of the $\mathrm{SU}(3)_{c}$ gauge fields, i.e. gluons, are partially neglected, as we discard the operators $H_{r s}^{\alpha} H_{r s}^{\alpha},\left(C_{0}^{\alpha} C_{0}^{\alpha}\right)^{2}, A_{0}^{a} A_{0}^{a} C_{0}^{\alpha} C_{0}^{\alpha}$ and $B_{0}^{2} C_{0}^{\alpha} C_{0}^{\alpha}$ from the effective theory for the heavy scale. Spatial gluons do not couple to the scalar field, while the self interactions of temporal gluons and their interactions with other adjoint fields would have a very small contribution to our quantities of interest, such as the scalar mass parameter of the effective theory for the light scale, cf. section 3.4.

- In the superheavy $\sigma$ case, a momentum-dependent four-point self-interaction of the Higgs doublet is generated through the $\sigma$-exchange diagram shown in eq. (2.25). To see this, simply expand the $\sigma$ propagator in powers of momentum, or equivalently solve the equation of motion of $\sigma$ including just its kinetic term and the $\mu_{m}$ coupling. This yields an induced interaction for the Higgs,

$$
\mathscr{L}_{\text {ind }}=-\frac{1}{8} \mu_{m}^{2}\left(\phi^{\dagger} \phi\right) \frac{1}{-\square+\mu_{\sigma}^{2}}\left(\phi^{\dagger} \phi\right) \text {. }
$$

From an expansion in powers of derivatives, one gets an infinite series of interactions. Since $\mu_{\sigma}$ is of order $g^{0}$ while the momentum in the effective theory for the heavy scale is of order $g^{1}$, the expansion starts at order $g^{2}$. Every power of $\square$ then adds an extra factor of $g^{2}$. The first operator containing derivatives, $\left(\phi^{\dagger} \phi\right) \square\left(\phi^{\dagger} \phi\right)$, therefore comes with an order- $g^{4}$ coefficient, which is safe to neglect to the order at which we work.

- The first non-derivative self-coupling of the Higgs doublet, not included in our effective theory, namely $\left(\phi^{\dagger} \phi\right)^{3}$, receives a contribution proportional to $\mu_{3} \mu_{m}^{3} \sim g^{4}$, generated by the tree-level diagram

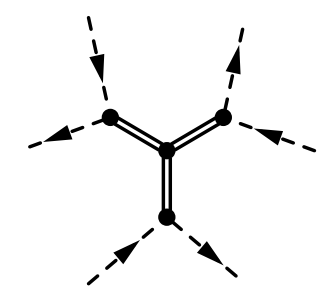

in the superheavy $\sigma$ case. While this is dominant over the contributions to the same operator from the SM superheavy fields, which only start at order $g^{6}$, it will be likewise neglected in our analysis carried out below.

\section{Dimensional reduction in the superheavy $\sigma$ case}

In this section, we perform the dimensional reduction step for a superheavy singlet scalar. This requires explicitly computing a set of Green's functions in both the full and the effective theory, requiring that the results agree at distances of order $1 /(g T)$. The calculations are divided into three parts: in section 3.1, we list the results for the necessary two- and four-point graphs; in section 3.2, we review the explicit counterterms needed; and in section 3.3, we use these to derive results for the parameters of the effective theory.

The discussion of the present section follows closely that of the dimensional reduction in the Standard Model performed in ref. [13]. In the main text, we only highlight explicitly 
contributions from Feynman diagrams that are new compared to the Standard Model, that is, those that involve at least one $\sigma$ propagator. For the sake of completeness, the results for all SM Feynman diagrams contributing to the effective theory parameters are listed in appendix C. Note that, in contrast to ref. [13], we do not make the scaling assumption $g^{\prime} \sim g^{3 / 2}$. Consequently, we must consider a group of SM diagrams that were neglected in that work.

\subsection{Correlators for the dimensional reduction}

We start by calculating a set of correlators in the full four-dimensional theory. The results listed below are given in terms of a set of master sum-integrals introduced in appendix B. Special attention is paid to subtleties related to the assumed superheavy nature of $\sigma$ : apart from having to deal with massive $\sigma$ propagators, a major modification is that we also need to include graphs which are one- $\sigma$-reducible. Led by practical convenience, we evaluate the contributions to wavefunction renormalization and to the interaction vertices of the temporal gauge fields by a direct diagrammatic analysis. The correlators in the scalar sector, on the other hand, are determined afterwards using the effective potential.

\subsubsection{Self-energy diagrams}

We start by considering the two-point functions. In order to be able to extract the contributions to both the kinetic terms and the mass parameters of the fields, we expand the correlators to second order in the external momentum $P$.

$\mathrm{SU}(2)_{L}$ gauge boson self-energy.

$a \mu \sim \sim b \nu$

$$
\begin{aligned}
= & g^{2} \delta_{a b}\left[-(d-1)(2 d-1) I_{1}^{4 b}+\frac{1}{6}\left(16-3 d+2 d^{2}\right) P^{2} I_{2}^{4 b}\right] \\
& +g^{2} \delta_{a b}(d-1) N_{f}\left(1+N_{c}\right)\left[\left(2^{2-d}-1\right) I_{1}^{4 b}-\frac{1}{6}\left(2^{4-d}-1\right) P^{2} I_{2}^{4 b}\right]
\end{aligned}
$$

for $\mu=\nu=0$,

$$
=g^{2} \delta_{a b}\left[\frac{1}{6}(31-2 d)-\frac{1}{3}\left(2^{4-d}-1\right) N_{f}\left(1+N_{c}\right)\right]\left(\delta_{r s} P^{2}-P_{r} P_{s}\right) I_{2}^{4 b}
$$

for $\mu=r, \nu=s$.

\section{$\mathrm{U}(1)_{Y}$ gauge boson self-energy.}

$\mu$ WUYW $\nu$

$$
\begin{aligned}
= & g^{\prime 2}\left[(1-d) I_{1}^{4 b}-\frac{2}{3}\left(1-\frac{d}{4}\right) P^{2} I_{2}^{4 b}\right]-\frac{1}{2} g^{\prime 2}(d-1) N_{f} \\
& \times\left[2 Y_{\ell}^{2}+Y_{e}^{2}+N_{c}\left(2 Y_{q}^{2}+Y_{u}^{2}+Y_{d}^{2}\right)\right]\left[\left(1-2^{2-d}\right) I_{1}^{4 b}+\frac{1}{6}\left(2^{4-d}-1\right) P^{2} I_{2}^{4 b}\right]
\end{aligned}
$$

for $\mu=\nu=0$, 


$$
\begin{aligned}
= & -\frac{1}{6} g^{\prime 2}\left\{1+\left(2^{4-d}-1\right) N_{f}\left[2 Y_{\ell}^{2}+Y_{e}^{2}+N_{c}\left(2 Y_{q}^{2}+Y_{u}^{2}+Y_{d}^{2}\right)\right]\right\} \\
& \times\left(\delta_{r s} P^{2}-P_{r} P_{s}\right) I_{2}^{4 b}
\end{aligned}
$$

for $\mu=r, \nu=s$.

These two-point gauge-field correlators are not affected by $\sigma$ at one-loop order. In an analogous manner, we could determine the gluon Debye mass through the $\mathrm{SU}(3)_{c}$ gauge boson self energy, but instead we take it from the literature, cf. section 3.3.1. The wavefunction renormalization of the temporal gluon fields is not needed at all, as the temporal gluons do not couple to the Higgs field at tree level like the $\mathrm{SU}(2)_{L}$ and $\mathrm{U}(1)_{Y}$ gauge fields.

Higgs doublet self-energy. Here we only consider the contributions to wavefunction renormalization, i.e. the $P^{2}$ part of the correlator. (The corrections to the mass parameter will be extracted below from the effective potential.) In the Standard Model alone, there are three different one-loop diagrams that contribute to wavefunction renormalization, corresponding to the exchange of $A_{\mu}^{a}$ and $B_{\mu}$ and to the fermion loop, respectively. Altogether, they give

$$
\delta_{i j}\left\{\frac{9}{4} g^{2}+\frac{3}{4} g^{\prime 2}-\left(2^{4-d}-1\right) \operatorname{tr}\left[h^{(e)} h^{(e) \dagger}+N_{c} h^{(u)} h^{(u) \dagger}+N_{c} h^{(d)} h^{(d) \dagger}\right]\right\} P^{2} I_{2}^{4 b} .
$$

In addition, there is one diagram containing a massive $\sigma$ propagator:

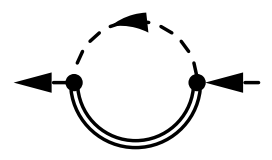

A brief calculation shows that the $P^{2}$ piece of this diagram reads ${ }^{3}$

$$
\frac{1}{4} \mu_{m}^{2} \delta_{i j} P^{2} \sum_{K} \frac{1}{K^{2}\left(K^{2}+\mu_{\sigma}^{2}\right)^{2}}\left(\frac{4}{d} \frac{k^{2}}{K^{2}+\mu_{\sigma}^{2}}-1\right)=\frac{1}{4} \mu_{m}^{2} \delta_{i j} P^{2}\left[\frac{4}{d} \tilde{J}_{3 / 1,0,1}^{4 b}\left(\mu_{\sigma}\right)-\tilde{J}_{2 / 1}^{4 b}\left(\mu_{\sigma}\right)\right] .
$$

Note that this sum-integral is manifestly finite and thus does not require any regularization. Also, unlike the sum-integrals with SM propagators only, the zero mode $i s$ included here.

\subsubsection{Correlators with gauge fields}

We consider first the self-couplings of the temporal gauge fields. At one loop, these do not receive any contributions from the $\sigma$ field, and we therefore merely list the results.

The $A_{0}^{a} A_{0}^{b} A_{0}^{c} A_{0}^{d}$ correlator.

$$
\left\{\begin{array}{l}
=\frac{1}{6}(d-1)(d-3)\left[8 d-7+\left(1-2^{4-d}\right) N_{f}\left(1+N_{c}\right)\right] \\
\times g^{4}\left(\delta_{a b} \delta_{c d}+\delta_{a c} \delta_{b d}+\delta_{a d} \delta_{b c}\right) I_{2}^{4 b} .
\end{array}\right.
$$

\footnotetext{
${ }^{3}$ In our notation four-momenta are written $K=\left(K_{0}, \boldsymbol{k}\right)$; see appendix B.
} 


\section{The $B_{0}^{4}$ correlator.}

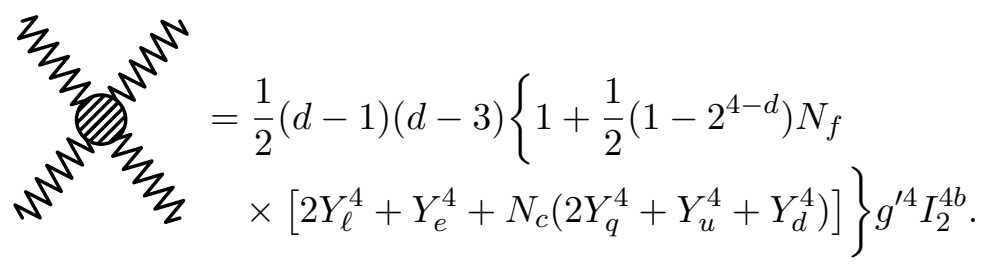

The $A_{0}^{a} A_{0}^{b} B_{0}^{2}$ correlator.

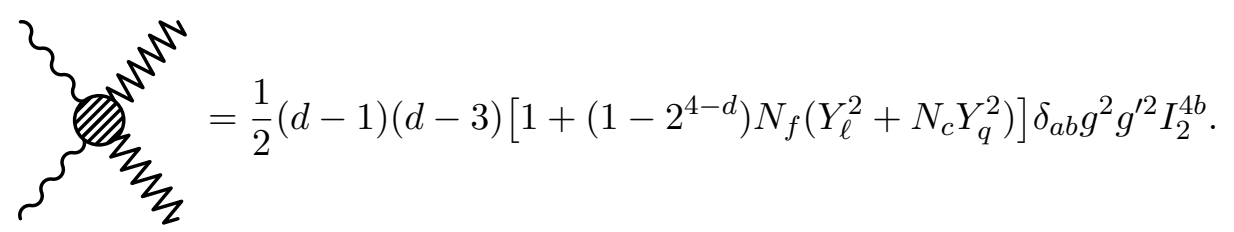

Next, we consider the four-point functions with two gauge field and two scalar legs. Knowing the wavefunction renormalization factors of all the fields, the correlators with temporal gauge fields determine the new couplings of these fields in the three-dimensional effective theory, whereas the correlators with spatial gauge fields determine the gauge couplings $g_{3}$ and $g_{3}^{\prime}$. In principle, the same gauge couplings can also be extracted from four-point gauge correlators, which are however somewhat more difficult to evaluate. The correlators used here, albeit simpler to calculate, have a downside: they contain explicit contributions from $\sigma$, which in the final expressions for $g_{3}$ and $g_{3}^{\prime}$ have to cancel against similar contributions coming from the Higgs field wavefunction renormalization. ${ }^{4}$ We consider this a nontrivial test of the correctness of our calculation.

The $\phi^{\dagger i} \phi^{j} A_{\mu}^{a} A_{\nu}^{b}$ correlator. We first put together all 1PI diagrams without any $\sigma$ propagators, getting

$$
\begin{aligned}
& \delta_{i j} \delta_{a b}\left\{d\left(d-\frac{25}{8}\right) g^{4}+\frac{d}{8} g^{2} g^{\prime 2}+3(d-3) \lambda_{h} g^{2}\right. \\
& \left.+\frac{1}{2}\left(2^{4-d}-1\right)(2-d) g^{2} \operatorname{tr}\left[h^{(e)} h^{(e) \dagger}+N_{c} h^{(u)} h^{(u) \dagger}+N_{c} h^{(d)} h^{(d) \dagger}\right]\right\} I_{2}^{4 b}
\end{aligned}
$$

for $\mu=\nu=0$,

$$
\delta_{i j} \delta_{a b} \delta_{r s}\left\{-\frac{3}{8} g^{4}+\frac{3}{8} g^{2} g^{\prime 2}-\frac{1}{2}\left(2^{4-d}-1\right) g^{2} \operatorname{tr}\left[h^{(e)} h^{(e) \dagger}+N_{c} h^{(u)} h^{(u) \dagger}+N_{c} h^{(d)} h^{(d) \dagger}\right]\right\} I_{2}^{4 b}
$$

for $\mu=r, \nu=s$.

In addition, there are two one- $\sigma$-irreducible $(1 \sigma \mathrm{I})$ and two one- $\sigma$-reducible $(1 \sigma \mathrm{R})$ diagrams which can be grouped into two pairs according to the coupling of the external gauge legs

\footnotetext{
${ }^{4}$ Since neither the two-point nor the four-point gauge correlators contain any $\sigma$ propagators at one loop, the effective theory gauge couplings are manifestly independent of $\sigma$.
} 
to the loop. The first pair reads

$$
\begin{aligned}
& \left\{\begin{array}{l}
3 \\
\}
\end{array}=-\frac{1}{8} \delta_{i j} \delta_{a b} \delta_{\mu \nu} \mu_{m}^{2} g^{2} f_{K} \frac{1}{\left(K^{2}\right)^{2}}\left(\frac{1}{K^{2}+\mu_{\sigma}^{2}}+\frac{2}{\mu_{\sigma}^{2}}\right)\right. \\
& =-\frac{1}{8} \delta_{i j} \delta_{a b} \delta_{\mu \nu} \mu_{m}^{2} g^{2}\left[\tilde{J}_{1 / 2}^{4 b}\left(\mu_{\sigma}\right)+\frac{2}{\mu_{\sigma}^{2}} \tilde{I}_{2}^{4 b}\right] .
\end{aligned}
$$

Note that both sum-integrals contain an infrared divergence due to the presence of the zero Matsubara mode of a massless field. For diagrams without a $\sigma$ propagator, such divergences cancel straightforwardly in the matching against a contribution of the corresponding diagram in the three-dimensional theory, and can thus be dropped immediately. The treatment of diagrams with a $\sigma$ propagator is, however, more subtle since $\sigma$ is missing from the dimensionally reduced theory. This is resolved thanks to the tree-level self-interaction of the Higgs field, induced by a $\sigma$ exchange, cf. graph (2.25) and eq. (2.32). Its effect can be viewed as a modification of the quartic Higgs coupling $\lambda_{h}$. When inserted in the diagram (C.32) in the three-dimensional theory, this correction yields $-3 \delta_{i j} \delta_{a b} \delta_{\mu \nu} \mu_{m}^{2} g^{2} /\left(8 \mu_{\sigma}^{2}\right) \int_{k} \frac{1}{\left(k^{2}\right)^{2}}$, which is easily seen to cancel the infrared divergence in eq. (3.12). It is important to keep in mind that as a result of nonzero $\mu_{\sigma}$ in the $\sigma$ propagator, the zero mode contribution to eq. (3.12) contains a finite remainder even after the infrared divergence has been canceled, which has to be taken into account.

The other pair of diagrams with a $\sigma$ propagator reads
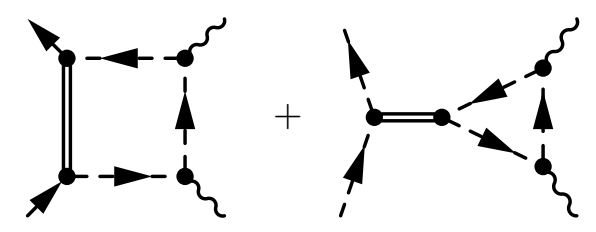

$$
\begin{aligned}
& =\frac{1}{2} \delta_{i j} \delta_{a b} \mu_{m}^{2} g^{2} \sum_{K} \frac{K_{\mu} K_{\nu}}{\left(K^{2}\right)^{3}}\left(\frac{1}{K^{2}+\mu_{\sigma}^{2}}+\frac{2}{\mu_{\sigma}^{2}}\right) \\
& =\frac{1}{2} \delta_{i j} \delta_{a b} \mu_{m}^{2} g^{2}\left[\tilde{J}_{1 / 3,1,0}^{4 b}\left(\mu_{\sigma}\right)+\frac{2}{\mu_{\sigma}^{2}} \tilde{I}_{3,1}^{4 b}\right]
\end{aligned}
$$

$$
\text { for } \mu=\nu=0 \text {, }
$$

$$
=\frac{1}{2 d} \delta_{i j} \delta_{a b} \delta_{r s} \mu_{m}^{2} g^{2}\left[\tilde{J}_{1 / 3,0,1}^{4 b}\left(\mu_{\sigma}\right)+\frac{2}{\mu_{\sigma}^{2}} \tilde{I}_{3,0,1}^{4 b}\right]
$$

for $\mu=r, \nu=s$.

The temporal part of this expression is infrared finite. The spatial part, however, has an infrared divergence. This is canceled by the mechanism described above, namely by inserting the $\sigma$-induced correction to $\lambda_{h}$ into the diagram (C.36) in the three-dimensional theory. Again, there is a finite leftover which must be evaluated properly.

The $\phi^{\dagger i} \phi^{j} \boldsymbol{B}_{\boldsymbol{\mu}} \boldsymbol{B}_{\boldsymbol{\nu}}$ correlator. This correlator is calculated following the same steps as above, albeit with different combinatorial factors. We first present the sum of all 1PI 
diagrams without a $\sigma$ propagator,

$$
\begin{aligned}
& \delta_{i j}\left(\frac{3}{8} d g^{2} g^{\prime 2}+\frac{d}{8} g^{\prime 4}+3(d-3) \lambda_{h} g^{\prime 2}-\frac{1}{2}\left(2^{4-d}-1\right) g^{\prime 2}\right. \\
& \times \operatorname{tr}\left\{(d-2)\left[\left(Y_{\ell}^{2}+Y_{e}^{2}\right) h^{(e)} h^{(e) \dagger}+N_{c}\left(Y_{q}^{2}+Y_{u}^{2}\right) h^{(u)} h^{(u) \dagger}+N_{c}\left(Y_{q}^{2}+Y_{d}^{2}\right) h^{(d)} h^{(d) \dagger}\right]\right. \\
& \left.\left.-2\left[Y_{e} Y_{\ell} h^{(e)} h^{(e) \dagger}+N_{c} Y_{u} Y_{q} h^{(u)} h^{(u) \dagger}+N_{c} Y_{d} Y_{q} h^{(d)} h^{(d) \dagger}\right]\right\}\right) I_{2}^{4 b}
\end{aligned}
$$

for $\mu=\nu=0$,

$$
\begin{aligned}
& \delta_{i j} \delta_{r s}\left\{\frac{9}{8} g^{2} g^{\prime 2}+\frac{3}{8} g^{\prime 4}-\frac{1}{2}\left(2^{4-d}-1\right) g^{\prime 2}\right. \\
& \left.\times \operatorname{tr}\left[\left(Y_{e}-Y_{\ell}\right)^{2} h^{(e)} h^{(e) \dagger}+N_{c}\left(Y_{u}-Y_{q}\right)^{2} h^{(u)} h^{(u) \dagger}+N_{c}\left(Y_{d}-Y_{q}\right)^{2} h^{(d)} h^{(d) \dagger}\right]\right\} I_{2}^{4 b}
\end{aligned}
$$

for $\mu=r, \nu=s$.

In addition to these, there are again two pairs of diagrams with a $\sigma$ propagator, which differ from those with $\mathrm{SU}(2)_{L}$ gauge boson lines just by replacing $g$ with $g^{\prime}$ and removing the overall factor $\delta_{a b}$. We collect the results here for completeness:

$$
\begin{gathered}
=\frac{1}{8} \delta_{i j} \delta_{\mu \nu} \mu_{m}^{2} g^{2}\left[\tilde{J}_{1 / 2}^{4 b}\left(\mu_{\sigma}\right)+\frac{2}{\mu_{\sigma}^{2}} \tilde{I}_{2}^{4 b}\right] \\
\text { for } \mu=r, \nu=s .
\end{gathered}
$$

Upon subtracting the contribution of the three-dimensional theory, there is again a finite leftover which must be carefully accounted for, and which expresses the contribution of the zero mode of $\sigma$ to the effective theory coupling.

The $\phi^{\dagger i} \phi^{j} \boldsymbol{A}_{\boldsymbol{\mu}}^{a} \boldsymbol{B}_{\boldsymbol{\nu}}$ correlator. Since the information about the gauge couplings in the three-dimensional theory can be extracted from the above correlators with two $A_{\mu}^{a}$ or two $B_{\mu}$ fields, we only need the temporal correlators, $\mu=\nu=0$, here. Putting first together all the diagrams without any $\sigma$ propagators gives

$$
\begin{aligned}
& \left(\tau_{a}\right)^{i j} I_{2}^{4 b}\left(\left[\frac{d}{8} g^{3} g^{\prime}+\frac{d}{8} g g^{\prime 3}+(d-3) \lambda_{h} g g^{\prime}\right]+\frac{1}{2}\left(1-2^{4-d}\right) g g^{\prime}\right. \\
& \left.\times \operatorname{tr}\left\{\left[(d-2) Y_{\ell}-Y_{e}\right] h^{(e)} h^{(e) \dagger}-N_{c}\left[(d-2) Y_{q}-Y_{u}\right] h^{(u)} h^{(u) \dagger}+N_{c}\left[(d-2) Y_{q}-Y_{d}\right] h^{(d)} h^{(d) \dagger}\right\}\right) .
\end{aligned}
$$


Next, we have to consider diagrams containing a $\sigma$ propagator, and there is again a oneto-one correspondence between the $1 \sigma \mathrm{I}$ and $1 \sigma \mathrm{R}$ graphs. First, we find

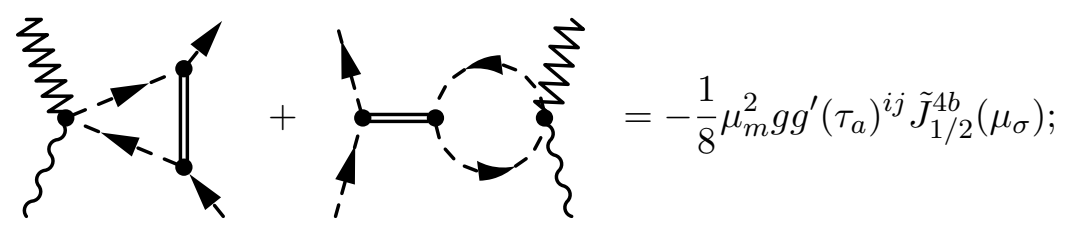

the latter diagram vanishes thanks to the trace in the scalar doublet loop. The same is true for the other $1 \sigma \mathrm{R}$ diagrams, and we therefore only show here the $1 \sigma \mathrm{I}$ graphs which give a nontrivial contribution, namely

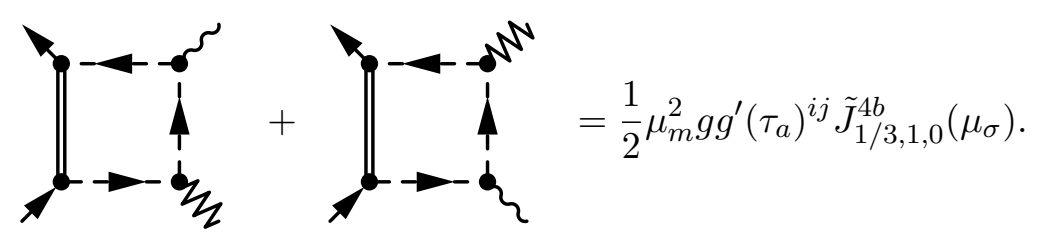

The $\phi^{\dagger i} \phi^{j} C_{0}^{\alpha} C_{0}^{\beta}$ correlator.

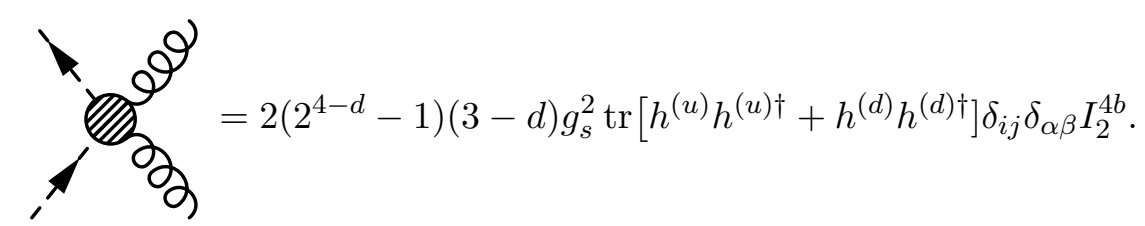

\subsubsection{Effective potential for the scalars}

The correlators in the scalar sector are comprised of a large number of diagrams even at the one-loop level. It is therefore advantageous to obtain the corresponding operators in the effective theory using the effective potential method. To that end, we shift the scalar fields by their assumed expectation values,

$$
\left\langle\phi^{i}\right\rangle \equiv \varphi^{i}, \quad\langle\sigma\rangle \equiv \rho .
$$

The shift affects the $\mathscr{L}_{\text {scalar }}$ and $\mathscr{L}_{\text {Yukawa }}$ parts of the Lagrangian (2.1). For $\mathscr{L}_{\text {Yukawa }}$, the shift simply results in a number of mass terms for the fermions. On the other hand the shift of the scalar fields has a twofold effect on $\mathscr{L}_{\text {scalar }}$. First, it leads to a modification of some of the couplings in the form $\mu_{i} \rightarrow \tilde{\mu}_{i}$, $\operatorname{with}^{5}$

$$
\begin{array}{llrl}
\tilde{\mu}_{h}^{2} & =\mu_{h}^{2}-2 \lambda_{h} \varphi^{\dagger} \varphi-\frac{1}{2} \mu_{m} \rho-\frac{1}{2} \lambda_{m} \rho^{2}, & \tilde{\mu}_{3}=\mu_{3}+3 \lambda_{\sigma} \rho, \\
\tilde{\mu}_{\sigma}^{2}=\mu_{\sigma}^{2}+2 \mu_{3} \rho+3 \lambda_{\sigma} \rho^{2}+\lambda_{m} \varphi^{\dagger} \varphi, & \tilde{\mu}_{m}=\mu_{m}+2 \lambda_{m} \rho .
\end{array}
$$

Second, it produces a number of new operators that do not appear in the original Lagrangian. There are several new interaction vertices, encoded in

$$
\begin{aligned}
\mathscr{L}_{\text {int }}^{\text {new }}= & \frac{1}{4}\left(g^{2} \vec{A}_{\mu} \cdot \vec{A}_{\mu}+g^{\prime 2} B_{\mu}^{2}\right)\left(\varphi^{\dagger} \phi+\phi^{\dagger} \varphi\right)+\frac{1}{2} g g^{\prime} B_{\mu} \vec{A}_{\mu} \cdot\left(\varphi^{\dagger} \vec{\tau} \phi+\phi^{\dagger} \vec{\tau} \varphi\right) \\
& +2 \lambda_{h} \phi^{\dagger} \phi\left(\phi^{\dagger} \varphi+\varphi^{\dagger} \phi\right)+\frac{1}{2} \lambda_{m} \sigma^{2}\left(\phi^{\dagger} \varphi+\varphi^{\dagger} \phi\right),
\end{aligned}
$$

\footnotetext{
${ }^{5}$ The modified linear coupling $\tilde{\mu}_{1}$ is not needed for the calculation of the effective potential, and thus is not given here explicitly.
} 
and in addition a number of new bilinear terms which introduce mixings between the fields. Together with the already existing bilinear terms for the gauge fields and the scalars, these can be written in the form

$$
\begin{aligned}
\mathscr{L}_{\text {bilin }}= & \frac{1}{4} G_{\mu \nu}^{a} G_{\mu \nu}^{a}+\frac{1}{4} F_{\mu \nu} F_{\mu \nu}+\frac{1}{4}\left(g^{2} \vec{A}_{\mu} \cdot \vec{A}_{\mu}+g^{\prime 2} B_{\mu}^{2}\right) \varphi^{\dagger} \varphi+\frac{1}{2} g g^{\prime} B_{\mu} \vec{A}_{\mu} \cdot \varphi^{\dagger} \vec{\tau} \varphi \\
& +\partial_{\mu} \phi^{\dagger} \partial_{\mu} \phi-\tilde{\mu}_{h}^{2} \phi^{\dagger} \phi+\frac{1}{2}\left(\partial_{\mu} \sigma\right)^{2}+\frac{1}{2} \tilde{\mu}_{\sigma}^{2} \sigma^{2}+\left(\frac{1}{2} \mu_{m}+\lambda_{m} \rho\right) \sigma\left(\phi^{\dagger} \varphi+\varphi^{\dagger} \phi\right) \\
& +\lambda_{h}\left(\phi^{\dagger} \varphi+\varphi^{\dagger} \phi\right)^{2}+\frac{\mathrm{i} g}{2} \vec{A}_{\mu} \cdot\left(\varphi^{\dagger} \vec{\tau} \partial_{\mu} \phi-\partial_{\mu} \phi^{\dagger} \vec{\tau} \varphi\right)+\frac{\mathrm{i} g^{\prime}}{2} B_{\mu}\left(\varphi^{\dagger} \partial_{\mu} \phi-\partial_{\mu} \phi^{\dagger} \varphi\right) .
\end{aligned}
$$

Note that the last two operators are irrelevant as they vanish when contracted with a gauge boson propagator in the Landau gauge. The bilinear part of the Lagrangian, $\mathscr{L}_{\text {bilin }}$ - together with a similar bilinear Lagrangian for the fermions - completely determines the effective potential at the one-loop level. Hence we only need to know the eigenvalues of the mass matrix for all the fields. Clearly, the masses of gluons as well as of all the ghosts are independent of $\varphi$ and $\rho$, and these fields therefore do not contribute to the effective potential (apart from its constant part, which does not play a role in the matching to the three-dimensional effective theory). In the electroweak gauge boson sector, the squared masses read

$$
0, \quad m_{W}^{2}=\frac{1}{2} g^{2} \varphi^{\dagger} \varphi \quad[2 \times], \quad m_{Z}^{2}=\frac{1}{2}\left(g^{2}+g^{\prime 2}\right) \varphi^{\dagger} \varphi
$$

In the scalar sector, we find three modes with mass squared $-\tilde{\mu}_{h}^{2}$. The remaining component of the Higgs doublet mixes with $\sigma$, and the mass eigenvalues have to be found by explicit diagonalization,

$$
m_{ \pm}^{2}=\frac{\tilde{\mu}_{\sigma}^{2}-\tilde{\mu}_{h}^{2}}{2}+2 \lambda_{h} \varphi^{\dagger} \varphi \pm \sqrt{\left(\frac{\tilde{\mu}_{\sigma}^{2}+\tilde{\mu}_{h}^{2}}{2}-2 \lambda_{h} \varphi^{\dagger} \varphi\right)^{2}+2 \varphi^{\dagger} \varphi\left(\frac{1}{2} \mu_{m}+\lambda_{m} \rho\right)^{2}} .
$$

The full one-loop effective potential of the four-dimensional theory then reads

$V_{\text {eff }}=d \tilde{K}^{4 b}\left(m_{Z}\right)+2 d \tilde{K}^{4 b}\left(m_{W}\right)+3 \tilde{K}^{4 b}\left(\mathrm{i} \tilde{\mu}_{h}\right)+\tilde{K}^{4 b}\left(m_{+}\right)+\tilde{K}^{4 b}\left(m_{-}\right)-4 \sum_{i} \tilde{K}^{4 f}\left(h_{i} \sqrt{\varphi^{\dagger} \varphi}\right)$,

where the sum runs over all eigenvalues of the Yukawa coupling matrices,

$$
h_{i} \in \operatorname{spectrum}\left(\sqrt{h^{(e)} h^{(e) \dagger}}, \sqrt{h^{(u)} h^{(u) \dagger}}, \sqrt{h^{(d)} h^{(d) \dagger}}\right)
$$

including the $N_{c}$-fold degeneracy due to different colors.

\subsubsection{Scalar correlators from the effective potential}

The 1PI correlators at zero momentum can be determined from the effective potential (3.27). As this is still an exact expression, we merely have to determine the scaling of individual couplings. Together with the tree-level potential, the result can be written using the generic notation

$$
V_{\mathrm{eff}}=V_{0,0}+V_{2,0} \varphi^{\dagger} \varphi+V_{4,0}\left(\varphi^{\dagger} \varphi\right)^{2}+V_{0,1} \rho+V_{0,2} \rho^{2}+V_{0,3} \rho^{3}+V_{0,4} \rho^{4}+V_{2,1} \rho \varphi^{\dagger} \varphi+\cdots,
$$


where the relevant coefficients read

$$
\begin{aligned}
V_{2,0}= & -\mu_{h}^{2}+\left(\frac{3}{4} d g^{2}+\frac{1}{4} d g^{\prime 2}+6 \lambda_{h}\right) I_{1}^{4 b}-2 \sum_{i} h_{i}^{2} I_{1}^{4 f}-\frac{1}{4} \mu_{m}^{2} \tilde{J}_{1 / 1}^{4 b}\left(\mu_{\sigma}\right)+\frac{1}{2} \lambda_{m} \tilde{J}_{1}^{4 b}\left(\mu_{\sigma}\right), \\
V_{4,0}= & \lambda_{h}-\left(\frac{3}{16} d g^{4}+\frac{1}{16} d g^{\prime 4}+\frac{1}{8} d g^{2} g^{\prime 2}+12 \lambda_{h}^{2}\right) I_{2}^{4 b}+\sum_{i} h_{i}^{4} I_{2}^{4 f} \\
& +\frac{3}{2} \lambda_{h} \mu_{m}^{2} \tilde{J}_{1 / 2}^{4 b}\left(\mu_{\sigma}\right)-\frac{1}{16} \mu_{m}^{4} \tilde{J}_{2 / 2}^{4 b}\left(\mu_{\sigma}\right)+\frac{1}{4} \lambda_{m} \mu_{m}^{2} \tilde{J}_{2 / 1}^{4 b}\left(\mu_{\sigma}\right)-\frac{1}{4} \lambda_{m}^{2} \tilde{J}_{2}^{4 b}\left(\mu_{\sigma}\right), \\
V_{0,1}= & \mu_{1}+\mu_{m} I_{1}^{4 b}+\mu_{3} \tilde{J}_{1}^{4 b}\left(\mu_{\sigma}\right), \\
V_{0,2}= & \frac{1}{2} \mu_{\sigma}^{2}+\lambda_{m} I_{1}^{4 b}-\frac{1}{4} \mu_{m}^{2} I_{2}^{4 b}-\mu_{3}^{2} \tilde{J}_{2}^{4 b}\left(\mu_{\sigma}\right)+\frac{3}{2} \lambda_{\sigma} \tilde{J}_{1}^{4 b}\left(\mu_{\sigma}\right), \\
V_{2,1}= & \frac{1}{2} \mu_{m}-3 \lambda_{h} \mu_{m} I_{2}^{4 b}+\frac{1}{2} \mu_{3} \mu_{m}^{2} \tilde{J}_{2 / 1}^{4 b}\left(\mu_{\sigma}\right)+\frac{1}{8} \mu_{m}^{3} \tilde{J}_{1 / 2}^{4 b}\left(\mu_{\sigma}\right)-\lambda_{m} \mu_{3} \tilde{J}_{2}^{4 b}\left(\mu_{\sigma}\right)-\lambda_{m} \mu_{m} \tilde{J}_{1 / 1}^{4 b}\left(\mu_{\sigma}\right) .
\end{aligned}
$$

This is, however, not quite enough. In order to match to the three-dimensional theory, we have to determine all one- $\phi$-irreducible contributions to the Higgs correlators. These include in particular diagrams that are $1 \sigma \mathrm{R}$.

We first consider the Higgs two-point function. The corresponding wavefunction renormalization was already found above, see eqs. (3.5) and (3.6). The static two-point function, on the other hand, consists of contributions of the forms

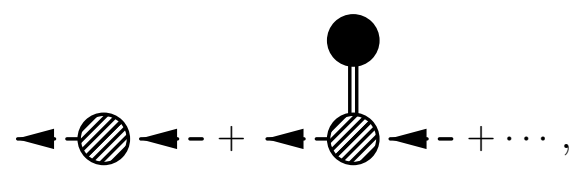

where the ellipsis stands for $1 \sigma \mathrm{R}$ diagrams carrying contributing of orders $g^{n}, n>2$. Note that the full circle denotes connected Green's functions, whereas the shaded circle the 1PI ones. To order $g^{2}$, we therefore obtain

$$
\begin{aligned}
\Pi_{2}= & -V_{2,0}+\frac{\mu_{m}}{2 \mu_{\sigma}^{2}} V_{0,1} \\
= & \mu_{h}^{2}+\frac{\mu_{1} \mu_{m}}{2 \mu_{\sigma}^{2}}-\left(\frac{3}{4} d g^{2}+\frac{1}{4} d g^{2}+6 \lambda_{h}\right) I_{1}^{4 b}+2 \sum_{i} h_{i}^{2} I_{1}^{4 f} \\
& +\frac{\mu_{3} \mu_{m}}{2 \mu_{\sigma}^{2}} \tilde{J}_{1}^{4 b}\left(\mu_{\sigma}\right)+\frac{1}{4} \mu_{m}^{2}\left[\tilde{J}_{1 / 1}^{4 b}\left(\mu_{\sigma}\right)+\frac{2 I_{1}^{4 b}}{\mu_{\sigma}^{2}}\right]-\frac{1}{2} \lambda_{m} \tilde{J}_{1}^{4 b}\left(\mu_{\sigma}\right) .
\end{aligned}
$$

The one- $\phi$-irreducible static four-point correlator is evaluated in a similar fashion. Symbolically, it is given by

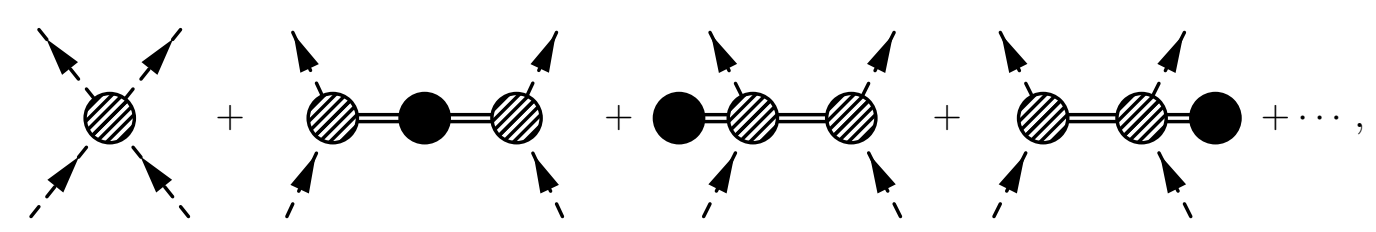

where the ellipsis now denotes contributions beyond order $g^{4}$. To compute this, we need to know the two-point function of $\sigma$. Fortunately, all the $\sigma$ propagators in these diagrams carry zero momentum so only the mass of $\sigma$ is needed. This is given by 


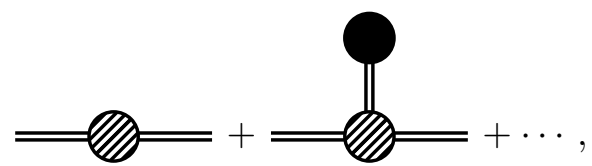

in a similar manner to eq. (3.31). As we are calculating the Higgs four-point function to order $g^{4}$ and the $\sigma \phi^{\dagger} \phi$ vertex begins at order $g$, we need to know the $\sigma$ mass to order $g^{2}$. Hence the one-point function of $\sigma$ is only needed to order $g$ and is given by $V_{0,1}$. Putting all the pieces together, we find the "renormalized squared $\sigma$ mass" to be

$$
\mu_{\sigma, \text { ren }}^{2}=2\left(V_{0,2}-\frac{\mu_{3}}{\mu_{\sigma}^{2}} V_{0,1}\right) .
$$

The full static four-point correlator of the Higgs field, indicated in eq. (3.33), then becomes $\left(\delta_{i k} \delta_{j \ell}+\delta_{i \ell} \delta_{j k}\right) \Pi_{4}$, where

$$
\begin{aligned}
\Pi_{4}= & -2 V_{4,0}+\frac{V_{2,1}^{2}}{\mu_{\sigma, \text { ren }}^{2}}-\frac{4}{\mu_{\sigma}^{4}} V_{0,1} V_{2,1} V_{2,2} \\
= & -2 \lambda_{h}+\frac{\mu_{m}^{2}}{4 \mu_{\sigma}^{2}}+\frac{\mu_{1} \mu_{3} \mu_{m}^{2}}{2 \mu_{\sigma}^{6}}-\frac{\mu_{1} \mu_{m} \lambda_{m}}{\mu_{\sigma}^{4}}+\left(\frac{3}{8} d g^{4}+\frac{1}{8} d g^{4}+\frac{1}{4} d g^{2} g^{2}+24 \lambda_{h}^{2}\right) I_{2}^{4 b} \\
& -2 \sum_{i} h_{i}^{4} I_{2}^{4 f}-\frac{3 \lambda_{h} \mu_{m}^{2}}{\mu_{\sigma}^{2}}\left[I_{2}^{4 b}+\mu_{\sigma}^{2} \tilde{J}_{1 / 2}^{4 b}\left(\mu_{\sigma}\right)\right]+\frac{\mu_{3} \mu_{m}^{3}}{2 \mu_{\sigma}^{6}}\left[I_{1}^{4 b}+\mu_{\sigma}^{4} \tilde{J}_{2 / 1}^{4 b}\left(\mu_{\sigma}\right)\right] \\
& +\frac{\mu_{3}^{2} \mu_{m}^{2}}{2 \mu_{\sigma}^{6}}\left[\tilde{J}_{1}^{4 b}\left(\mu_{\sigma}\right)+\mu_{\sigma}^{2} \tilde{J}_{2}^{4 b}\left(\mu_{\sigma}\right)\right]-\frac{\lambda_{m} \mu_{3} \mu_{m}}{\mu_{\sigma}^{4}}\left[\tilde{J}_{1}^{4 b}\left(\mu_{\sigma}\right)+\mu_{\sigma}^{2} \tilde{J}_{2}^{4 b}\left(\mu_{\sigma}\right)\right] \\
& -\frac{3 \lambda_{\sigma} \mu_{m}^{2}}{4 \mu_{\sigma}^{4}} \tilde{J}_{1}^{4 b}\left(\mu_{\sigma}\right)+\frac{\mu_{m}^{4}}{8 \mu_{\sigma}^{4}}\left[I_{2}^{4 b}+\mu_{\sigma}^{2} \tilde{J}_{1 / 2}^{4 b}\left(\mu_{\sigma}\right)+\mu_{\sigma}^{4} \tilde{J}_{2 / 2}^{4 b}\left(\mu_{\sigma}\right)\right] \\
& -\frac{\lambda_{m} \mu_{m}^{2}}{2 \mu_{\sigma}^{4}}\left[3 I_{1}^{4 b}+2 \mu_{\sigma}^{2} \tilde{J}_{1 / 1}^{4 b}\left(\mu_{\sigma}^{2}\right)+\mu_{\sigma}^{4} \tilde{J}_{2 / 1}^{4 b}\left(\mu_{\sigma}\right)\right]+\frac{1}{2} \lambda_{m}^{2} \tilde{J}_{2}^{4 b}\left(\mu_{\sigma}\right) .
\end{aligned}
$$

\subsection{Counterterms and $\beta$-functions}

All the counterterms of the SSM are defined in section 2.1.1. We use the modified minimal subtraction $(\overline{\mathrm{MS}})$ scheme. When implemented in combination with dimensional regularization and the definition of momentum integrals according to eq. (B.1), finding the counterterms then amounts to extracting the pole part of the corresponding correlators. ${ }^{6}$ From this point on, we make the substitution $N_{c}=3$ in the main text. The field renormalization counterterms can be extracted from the two-point correlators, obtained in section 3.1.1, giving

$$
\begin{aligned}
& \delta Z_{A}=\frac{g^{2}}{16 \pi^{2} \epsilon}\left[\frac{25}{6}-\frac{4}{3} N_{f}\right], \\
& \delta Z_{B}=-\frac{g^{\prime 2}}{96 \pi^{2} \epsilon}\left\{1+N_{f}\left[2 Y_{\ell}^{2}+Y_{e}^{2}+3\left(2 Y_{q}^{2}+Y_{u}^{2}+Y_{d}^{2}\right)\right]\right\}, \\
& \delta Z_{\phi}=\frac{1}{16 \pi^{2} \epsilon}\left\{\frac{9}{4} g^{2}+\frac{3}{4} g^{\prime 2}-\operatorname{tr}\left[h^{(e)} h^{(e) \dagger}+3 h^{(u)} h^{(u) \dagger}+3 h^{(d)} h^{(d) \dagger}\right]\right\} .
\end{aligned}
$$

\footnotetext{
${ }^{6}$ We note that the ultraviolet divergences are independent of temperature, hence the counterterms can be extracted from correlators computed either at nonzero temperature or in the vacuum. We can, and will, therefore make use of the previously calculated thermal correlators.
} 
The $\sigma$ field receives no divergent contribution to wavefunction renormalization at one loop, and hence $Z_{\sigma}=1$. The counterterms for the gauge and Yukawa couplings are not affected by $\sigma$ at one loop, and thus agree with the SM results. We list here the results, valid in Landau gauge and assuming that only the top quark Yukawa coupling is nonzero [13],

$$
\begin{aligned}
\delta g & =-\frac{g^{3}}{32 \pi^{2} \epsilon}\left(\frac{43}{6}-\frac{4}{3} N_{f}\right) \\
\delta g^{\prime} & =\frac{g^{\prime 3}}{192 \pi^{2} \epsilon}\left(1+\frac{40}{3} N_{f}\right) \\
\delta g_{Y} & =\frac{g_{Y}}{16 \pi^{2} \epsilon}\left(\frac{9}{4} g_{Y}^{2}-\frac{9}{8} g^{2}-\frac{17}{24} g^{\prime 2}-4 g_{s}^{2}\right) .
\end{aligned}
$$

The counterterms for the couplings in the scalar sector can be found using the one-loop effective potential, calculated in section 3.1.3. We take eq. (3.27) and expand the integrals around the actual mass parameters of the fields in powers of the classical fields $\varphi$ and $\rho$. The individual counterterms are then readily identified as

$$
\begin{aligned}
\delta \mu_{h}^{2} & =\frac{1}{64 \pi^{2} \epsilon}\left(24 \lambda_{h} \mu_{h}^{2}-\mu_{m}^{2}-2 \lambda_{m} \mu_{\sigma}^{2}\right) \\
\delta \lambda_{h} & =\frac{1}{256 \pi^{2} \epsilon}\left(9 g^{4}+3 g^{\prime 4}+6 g^{2} g^{\prime 2}-48 g_{Y}^{4}+192 \lambda_{h}^{2}+4 \lambda_{m}^{2}\right) \\
\delta \mu_{\sigma}^{2} & =\frac{1}{32 \pi^{2} \epsilon}\left(4 \mu_{3}^{2}+\mu_{m}^{2}+6 \lambda_{\sigma} \mu_{\sigma}^{2}-4 \lambda_{m} \mu_{h}^{2}\right), \\
\delta \mu_{1} & =-\frac{1}{16 \pi^{2} \epsilon}\left(\mu_{h}^{2} \mu_{m}-\mu_{\sigma}^{2} \mu_{3}\right) \\
\delta \mu_{3} & =\frac{3}{32 \pi^{2} \epsilon}\left(6 \lambda_{\sigma} \mu_{3}+\lambda_{m} \mu_{m}\right) \\
\delta \lambda_{\sigma} & =\frac{1}{16 \pi^{2} \epsilon}\left(\lambda_{m}^{2}+9 \lambda_{\sigma}^{2}\right) \\
\delta \mu_{m} & =\frac{1}{8 \pi^{2} \epsilon}\left[3 \lambda_{h} \mu_{m}+\lambda_{m}\left(\mu_{3}+\mu_{m}\right)\right] \\
\delta \lambda_{m} & =\frac{1}{16 \pi^{2} \epsilon} \lambda_{m}\left(6 \lambda_{h}+2 \lambda_{m}+3 \lambda_{\sigma}\right) .
\end{aligned}
$$

The wavefunction renormalization factors together with the coupling counterterms determine, in the usual manner, the running of the couplings with renormalization scale $\Lambda$. From the one-loop counterterms listed above, we obtain the one-loop $\beta$-functions of all the couplings of the SSM:

$$
\begin{aligned}
& \Lambda \frac{d}{d \Lambda} g^{2}=-\frac{g^{4}}{8 \pi^{2}}\left(\frac{43}{6}-\frac{4}{3} N_{f}\right) \\
& \Lambda \frac{d}{d \Lambda} g^{\prime 2}=\frac{g^{\prime 4}}{8 \pi^{2}}\left(\frac{1}{6}+\frac{20}{9} N_{f}\right) \\
& \Lambda \frac{d}{d \Lambda} g_{Y}^{2}=\frac{1}{8 \pi^{2}}\left(\frac{9}{2} g_{Y}^{4}-8 g_{s}^{2} g_{Y}^{2}-\frac{9}{4} g^{2} g_{Y}^{2}-\frac{17}{12} g^{\prime 2} g_{Y}^{2}\right) \\
& \Lambda \frac{d}{d \Lambda} \mu_{h}^{2}=\frac{1}{8 \pi^{2}}\left[-\frac{\mu_{m}^{2}}{4}-\frac{1}{2} \lambda_{m} \mu_{\sigma}^{2}+\mu_{h}^{2}\left(-\frac{9}{4} g^{2}-\frac{3}{4} g^{\prime 2}+6 \lambda_{h}+g_{Y, 1}^{2}\right)\right]
\end{aligned}
$$




$$
\begin{aligned}
& \Lambda \frac{d}{d \Lambda} \lambda_{h}=\frac{1}{8 \pi^{2}} {\left[12 \lambda_{h}^{2}+\frac{1}{4} \lambda_{m}^{2}+\frac{9}{16} g^{4}+\frac{3}{8} g^{2} g^{\prime 2}+\frac{3}{16} g^{\prime 4}-\sum_{i} h_{i}^{4}\right.} \\
&\left.\quad-\lambda_{h} \frac{3}{2}\left(3 g^{2}+g^{\prime 2}\right)+2 \lambda_{h} g_{Y, 1}^{2}\right], \\
& \Lambda \frac{d}{d \Lambda} \mu_{\sigma}^{2}=\frac{1}{8 \pi^{2}}\left(2 \mu_{3}^{2}+\frac{1}{2} \mu_{m}^{2}+3 \lambda_{\sigma} \mu_{\sigma}^{2}-2 \lambda_{m} \mu_{h}^{2}\right), \\
& \Lambda \frac{d}{d \Lambda} \mu_{1}=\frac{1}{8 \pi^{2}}\left(\mu_{3} \mu_{\sigma}^{2}-\mu_{h}^{2} \mu_{m}\right), \\
& \Lambda \frac{d}{d \Lambda} \mu_{3}=\frac{3}{8 \pi^{2}}\left(3 \lambda_{\sigma} \mu_{3}+\frac{1}{2} \lambda_{m} \mu_{m}\right) \\
& \Lambda \frac{d}{d \Lambda} \lambda_{\sigma}=\frac{1}{8 \pi^{2}}\left(\lambda_{m}^{2}+9 \lambda_{\sigma}^{2}\right), \\
& \Lambda \frac{d}{d \Lambda} \mu_{m}=\frac{1}{8 \pi^{2}}\left[2 \lambda_{m} \mu_{3}+\mu_{m}\left(-\frac{9}{4} g^{2}-\frac{3}{4} g^{\prime 2}+6 \lambda_{h}+2 \lambda_{m}+g_{Y, 1}^{2}\right)\right], \quad \text { and } \\
& \Lambda \frac{d}{d \Lambda} \lambda_{m}=\frac{\lambda_{m}}{8 \pi^{2}}\left(-\frac{9}{4} g^{2}-\frac{3}{4} g^{\prime 2}+g_{Y, 1}^{2}+2 \lambda_{m}+6 \lambda_{h}+3 \lambda_{\sigma}\right) .
\end{aligned}
$$

Note that the running of the strong coupling $g_{s}$ is not included here, as it is not needed at the order of our calculation due to the fact that the parameter does not appear in any tree-level results.

\subsection{Matching relations}

Having considered above both the correlators needed for dimensional reduction as well as all the required counterterms, we are ready to move to the explicit derivation of the effective theory parameters. For the heavy scale effective theory, these are obtained by matching the long-distance behavior of various static Green's functions with the full theory. The final step to the effective theory for the light scale is described below in section 3.4.

\subsubsection{Thermal masses and normalization of fields}

We start with the mass parameters for the temporal gauge fields. These are forbidden in the four-dimensional theory by gauge invariance, and therefore arise solely from integration of the superheavy modes. To leading order in powers of the gauge couplings, they can simply be read off the static limits of the two-point correlators, eqs. (3.1) and (3.3),

$$
\begin{aligned}
m_{D}^{2} & =g^{2}\left[(d-1)(2 d-1)-4 N_{f}(d-1)\left(2^{2-d}-1\right)\right] I_{1}^{4 b} \\
& =g^{2} T^{2}\left(\frac{5}{6}+\frac{N_{f}}{3}\right) \\
m_{D}^{\prime 2} & =g^{\prime 2}(d-1)\left\{1-\frac{1}{2} N_{f}\left[2 Y_{l}^{2}+Y_{e}^{2}+3\left(2 Y_{q}^{2}+Y_{u}^{2}+Y_{d}^{2}\right)\right]\left(2^{2-d}-1\right)\right\} I_{1}^{4 b} \\
& =g^{\prime 2} T^{2}\left(\frac{1}{6}+\frac{5 N_{f}}{9}\right) .
\end{aligned}
$$

The gluon Debye mass $m_{D}^{\prime \prime}$ can be taken from the literature, see e.g. ref. [55]

$$
m_{D}^{\prime \prime 2}=g_{s}^{2} T^{2}\left(1+\frac{N_{f}}{6}\right)
$$


For the evaluation of (most of) the other couplings of the effective theory, we need to know the relation between the three-dimensional and the four-dimensional fields. Within this section, these will be distinguished by the lower indices $3 \mathrm{~d}$ and $4 \mathrm{~d}$, respectively. For a generic field, the relation between the fields reads

$$
\psi_{3 \mathrm{~d}}^{2}=\frac{1}{T}\left[1+\Pi_{\psi}^{\prime}(0)-\delta Z_{\psi}\right] \psi_{4 \mathrm{~d}}^{2},
$$

where $\Pi_{\psi}(P)$ is the self-energy of the field, a prime denotes a derivative with respect to $P^{2}$, and $\delta Z_{\psi}$ is the field renormalization counterterm. We will now consider all the fields of the three-dimensional effective theory one by one.

The SU(2) ${ }_{L}$ gauge fields. Using the momentum-dependent parts of eqs. (3.1) and (3.2) as well as the counterterm from eq. (3.37), the general relation (3.54) immediately leads to

$$
\begin{aligned}
& A_{3 \mathrm{~d}, 0}^{2}=\frac{A_{4 \mathrm{~d}, 0}^{2}}{T}\left\{1+\frac{g^{2}}{(4 \pi)^{2}}\left[-\frac{25 L_{b}}{6}+3+\frac{4 N_{f}}{3}\left(L_{f}-1\right)\right]\right\}, \\
& A_{3 \mathrm{~d}, r}^{2}=\frac{A_{4 \mathrm{~d}, r}^{2}}{T}\left[1+\frac{g^{2}}{(4 \pi)^{2}}\left(-\frac{25 L_{b}}{6}-\frac{2}{3}+\frac{4 N_{f}}{3} L_{f}\right)\right],
\end{aligned}
$$

where we have followed ref. [13] in defining

$$
L_{b} \equiv 2 \log \left(\frac{\Lambda}{4 \pi T}\right)+2 \gamma, \quad L_{f} \equiv L_{b}+4 \log 2 .
$$

Note that the divergences coming from the two-point correlators and the wavefunction renormalization factors have to cancel each other in the final matching relations for the fields. This is another nontrivial check that our calculation is correct.

The $\mathrm{U}(\mathbf{1})_{Y}$ gauge fields. Here we analogously use the momentum-dependent parts of eqs. (3.3) and (3.4) in combination with the counterterm from eq. (3.37) to get

$$
\begin{aligned}
& B_{3 \mathrm{~d}, 0}^{2}=\frac{B_{4 \mathrm{~d}, 0}^{2}}{T}\left\{1+\frac{g^{\prime 2}}{(4 \pi)^{2}}\left[\frac{L_{b}}{6}+\frac{1}{3}+\frac{20 N_{f}}{9}\left(L_{f}-1\right)\right]\right\}, \\
& B_{3 \mathrm{~d}, r}^{2}=\frac{B_{4 \mathrm{~d}, r}^{2}}{T}\left[1+\frac{g^{\prime 2}}{(4 \pi)^{2}}\left(\frac{L_{b}}{6}+\frac{20 N_{f}}{9} L_{f}\right)\right] .
\end{aligned}
$$

The Higgs field. This is the first case where the effects of the new scalar $\sigma$ contribute. Following the same steps as for the gauge fields, we combine eqs. (3.5) and (3.6) with the counterterm from eq. (3.37) to get

$$
\left(\phi^{\dagger} \phi\right)_{3 \mathrm{~d}}=\frac{\left(\phi^{\dagger} \phi\right)_{4 \mathrm{~d}}}{T}\left[1-\frac{3}{4(4 \pi)^{2}}\left(3 g^{2}+g^{\prime 2}\right) L_{b}+\frac{g_{Y, 1}^{2}}{(4 \pi)^{2}} L_{f}-\frac{\mu_{m}^{2}}{4} \tilde{J}_{\Phi}\right],
$$

where we have defined

$$
\begin{aligned}
\tilde{J}_{\Phi} & \equiv \frac{4}{d} \tilde{J}_{3 / 1,0,1}^{4 b}\left(\mu_{\sigma}\right)-\tilde{J}_{2 / 1}^{4 b}\left(\mu_{\sigma}\right)=\frac{4}{3} \tilde{J}_{3 / 1,0,1}^{4 b}\left(\mu_{\sigma}\right)-\tilde{J}_{2 / 1}^{4 b}\left(\mu_{\sigma}\right), \\
g_{Y, 1}^{2} & \equiv \operatorname{tr}\left[h^{(e)} h^{(e) \dagger}+3 h^{(u)} h^{(u)^{\dagger}}+3 h^{(d)} h^{(d) \dagger}\right] \approx 3 g_{Y}^{2} .
\end{aligned}
$$

Note that $\tilde{J}_{\Phi}$ is finite and can be equivalently expressed as

$$
\tilde{J}_{\Phi}=-\frac{1}{32 \pi^{2} \mu_{\sigma}^{2}}-\frac{T^{2}}{12 \mu_{\sigma}^{4}}+\frac{2 \pi^{2} T^{4}}{45 \mu_{\sigma}^{6}}+\frac{J_{1}\left(\mu_{\sigma}\right)}{\mu_{\sigma}^{4}}+\frac{J_{2}\left(\mu_{\sigma}\right)}{\mu_{\sigma}^{2}}-\frac{4}{3}\left[\frac{J_{1,0,1}\left(\mu_{\sigma}\right)}{\mu_{\sigma}^{6}}+\frac{J_{2,0,1}\left(\mu_{\sigma}\right)}{\mu_{\sigma}^{4}}+\frac{J_{3,0,1}\left(\mu_{\sigma}\right)}{\mu_{\sigma}^{2}}\right] .
$$




\subsubsection{Coupling constants}

The couplings of the effective theory are obtained by matching the correlators computed in the three-dimensional and the four-dimensional theory. The calculation involves the correlators evaluated in section 3.1 as well the wavefunction renormalization and coupling counterterms listed in section 3.2. Note that the correlators computed in section 3.1 do not include the effects of wavefunction renormalization, and hence are to be treated as correlators of the bare four-dimensional fields.

The gauge couplings $\boldsymbol{g}_{\mathbf{3}}, \boldsymbol{g}_{\mathbf{3}}^{\prime}$. Let us illustrate the procedure by considering the $\mathrm{SU}(2)_{L}$ coupling $g_{3}$. We focus on the correlator with two Higgs legs and two spatial gauge field legs. Putting together the tree-level vertices with the results of eqs. (3.11), (3.12) and (3.13), equating the correlators in the three- and four-dimensional theories amounts to setting

$$
\begin{aligned}
& \phi_{3 \mathrm{~d}}^{\dagger i} \phi_{3 \mathrm{~d}}^{j} A_{3 \mathrm{~d}, r}^{a} A_{3 \mathrm{~d}, s}^{b}\left(-\frac{1}{2} g_{3}^{2} \delta_{i j} \delta_{a b} \delta_{r s}\right) \\
& =\frac{1}{T} \phi_{4 \mathrm{~d}(b)}^{\dagger i} \phi_{4 \mathrm{~d}(b)}^{j} A_{4 \mathrm{~d}, r(b)}^{a} A_{4 \mathrm{~d}, s(b)}^{b} \delta_{i j} \delta_{a b} \delta_{r s} \\
& \quad \times\left\{-\frac{1}{2}\left(g^{2}+\delta g^{2}\right)+\left[-\frac{3}{8} g^{4}+\frac{3}{8} g^{2} g^{\prime 2}-\frac{1}{2}\left(2^{4-d}-1\right) g^{2} g_{Y, 1}^{2}\right] I_{2}^{4 b}+\mu_{m}^{2} g^{2}\left[\tilde{J}_{\Phi A}^{(1)}+\tilde{J}_{\Phi A}^{(3)}\right]\right\},
\end{aligned}
$$

where we have defined

$$
\begin{aligned}
\tilde{J}_{\Phi A}^{(1)} & \equiv-\frac{1}{8}\left[\tilde{J}_{1 / 2}^{4 b}\left(\mu_{\sigma}\right)+\frac{2}{\mu_{\sigma}^{2}} I_{2}^{4 b}\right], \\
\tilde{J}_{\Phi A}^{(2)} & \equiv \frac{1}{2}\left[\tilde{J}_{1 / 3,1,0}^{4 b}\left(\mu_{\sigma}\right)+\frac{2}{\mu_{\sigma}^{2}}\left(1-\frac{d}{4}\right) I_{2}^{4 b}\right], \\
\tilde{J}_{\Phi A}^{(3)} & \equiv \frac{1}{2 d}\left[\tilde{J}_{1 / 3,0,1}^{4 b}\left(\mu_{\sigma}\right)+\frac{2}{\mu_{\sigma}^{2}} \frac{d}{4} I_{2}^{4 b}\right] .
\end{aligned}
$$

Note that the combination $\tilde{J}_{\Phi A}^{(1)}+\tilde{J}_{\Phi A}^{(2)}$ entering the above matching relation is ultraviolet finite. It is now a matter of simple algebra to put together the definitions of the bare fields in terms of the wavefunction renormalization factors, the coupling counterterm and the abovederived expressions for relations between the renormalized three-dimensional and fourdimensional fields. One arrives then at the final result for the three-dimensional coupling,

$$
g_{3}^{2}=g^{2}(\Lambda) T\left\{1+\frac{g^{2}}{(4 \pi)^{2}}\left(\frac{43}{6} L_{b}+\frac{2}{3}-\frac{4 N_{f}}{3} L_{f}\right)+\frac{\mu_{m}^{2}}{4} \tilde{J}_{\Phi}-2 \mu_{m}^{2}\left[\tilde{J}_{\Phi A}^{(1)}+\tilde{J}_{\Phi A}^{(3)}\right]\right\} .
$$

Here we have indicated explicitly the dependence of the four-dimensional coupling $g$ on the renormalization scale $\Lambda$. However, it is easy confirm with the renormalization flow equation (3.40) that $g_{3}$ is independent of $\Lambda$. The same comment applies to all the other three-dimensional couplings discussed below. Again, renormalization group independence represents a nontrivial check of the correctness of our calculation.

The evaluation of the coupling $g_{3}^{\prime}$ proceeds along the same lines, and we therefore just quote the final result,

$$
g_{3}^{\prime 2}=g^{\prime 2}(\Lambda) T\left\{1+\frac{g^{\prime 2}}{(4 \pi)^{2}}\left(-\frac{1}{6} L_{b}-\frac{20 N_{f}}{9} L_{f}\right)+\frac{\mu_{m}^{2}}{4} \tilde{J}_{\Phi}-2 \mu_{m}^{2}\left[\tilde{J}_{\Phi A}^{(1)}+\tilde{J}_{\Phi A}^{(3)}\right]\right\} .
$$


An interested reader can easily check this expression themselves, using eqs. (3.15) and (3.16). We note that both $g_{3}$ and $g_{3}^{\prime}$ depend on the same combination of massive master integrals, which can be seen to vanish by an explicit manipulation,

$$
\frac{1}{4} \tilde{J}_{\Phi}-2\left[\tilde{J}_{\Phi A}^{(1)}+\tilde{J}_{\Phi A}^{(3)}\right]=\frac{1}{\mu_{\sigma}^{2}}\left\{\frac{J_{2}\left(\mu_{\sigma}\right)}{4}-\frac{J_{3,0,1}\left(\mu_{\sigma}\right)}{3}+\frac{1}{\mu_{\sigma}^{2}}\left[\frac{J_{1}\left(\mu_{\sigma}\right)}{2}-\frac{J_{2,0,1}\left(\mu_{\sigma}\right)}{3}\right]\right\}=0
$$

This is to be expected; we could have instead determined $g_{3}$ and $g_{3}^{\prime}$ using four-point correlators of the gauge fields, to which $\sigma$ does not contribute at the one-loop level.

The temporal gauge field self-couplings $\lambda_{3}, \lambda_{3}^{\prime}, \lambda_{3}^{\prime \prime}$. These couplings are forbidden by gauge invariance in the four-dimensional theory. They are generated at nonzero temperature by loop effects, and hence all appear at order $g^{4}$. Since we do not require higher precision in our setup, wavefunction renormalization does not contribute. Hence all the couplings can be straightforwardly obtained from the correlators of eqs. (3.7)-(3.9),

$$
\begin{aligned}
\lambda_{3} & =T \frac{g^{4}}{16 \pi^{2}} \frac{17-4 N_{f}}{3}, \\
\lambda_{3}^{\prime} & =T \frac{g^{\prime 4}}{16 \pi^{2}}\left[\frac{1}{3}-\frac{N_{f}}{6}\left(3 Y_{d}^{4}+Y_{e}^{4}+2 Y_{\ell}^{4}+6 Y_{q}^{4}+3 Y_{u}^{4}\right)\right] \\
& =T \frac{g^{\prime 4}}{16 \pi^{2}}\left(\frac{1}{3}-\frac{380}{81} N_{f}\right), \\
\lambda_{3}^{\prime \prime} & =T \frac{g^{2} g^{\prime 2}}{16 \pi^{2}}\left[2-2 N_{f}\left(Y_{\ell}^{2}+3 Y_{q}^{2}\right)\right]=T \frac{g^{2} g^{\prime 2}}{16 \pi^{2}}\left(2-\frac{8}{3} N_{f}\right) .
\end{aligned}
$$

The Higgs-gauge field couplings $\boldsymbol{h}_{\mathbf{3}}, \boldsymbol{h}_{\mathbf{3}}^{\prime}, \boldsymbol{h}_{\mathbf{3}}^{\prime \prime}, \boldsymbol{\delta}_{\mathbf{3}}$. These couplings are extracted from the correlators with two Higgs legs and two temporal gauge field legs. Putting together the results of eqs. (3.10), (3.12) and (3.13), equating the three-dimensional and the fourdimensional correlators amounts to setting

$$
\begin{aligned}
& \phi_{3 \mathrm{~d}}^{\dagger i} \phi_{3 \mathrm{~d}}^{j} A_{3 \mathrm{~d}, 0}^{a} A_{3 \mathrm{~d}, 0}^{b}\left(-2 h_{3} \delta_{i j} \delta_{a b}\right) \\
& =\frac{1}{T} \phi_{4 \mathrm{~d}(b)}^{\dagger i} \phi_{4 \mathrm{~d}(b)}^{j} A_{4 \mathrm{~d}, 0(b)}^{a} A_{4 \mathrm{~d}, 0(b)}^{b} \delta_{i j} \delta_{a b}\left\{-\frac{1}{2}\left(g^{2}+\delta g^{2}\right)+\mu_{m}^{2} g^{2}\left[\tilde{J}_{\Phi A}^{(1)}+\tilde{J}_{\Phi A}^{(2)}\right]\right. \\
& \left.\quad+\left[d\left(d-\frac{25}{8}\right) g^{4}+\frac{d}{8} g^{2} g^{\prime 2}+3(d-3) \lambda_{h} g^{2}+\frac{1}{2}\left(2^{4-d}-1\right)(2-d) g^{2} g_{Y, 1}^{2}\right] I_{2}^{4 b}\right\} .
\end{aligned}
$$

A straightforward calculation then leads to

$$
\begin{aligned}
h_{3}=\frac{g^{2}(\Lambda) T}{4}\left(1+\frac{1}{(4 \pi)^{2}}\left\{\left[\frac{43}{6} L_{b}+\frac{17}{2}-\frac{4 N_{f}}{3}\left(L_{f}-1\right)\right] g^{2}+\frac{g^{\prime 2}}{2}-2 g_{Y, 1}^{2}+12 \lambda_{h}\right\}\right. \\
\left.+\frac{\mu_{m}^{2}}{4} \tilde{J}_{\Phi}-2 \mu_{m}^{2}\left[\tilde{J}_{\Phi A}^{(1)}+\tilde{J}_{\Phi A}^{(2)}\right]\right) .
\end{aligned}
$$


The coupling $h_{3}^{\prime}$ is obtained from the correlator with two external $B_{0}$ legs, and its evaluation proceeds in exactly the same fashion. Using eqs. (3.14) and (3.16) yields

$$
\begin{aligned}
h_{3}^{\prime}=\frac{g^{\prime 2}(\Lambda) T}{4} & \left(1+\frac{1}{(4 \pi)^{2}}\left\{\frac{3 g^{2}}{2}+\left[-\frac{1}{6}\left(L_{b}-1\right)-\frac{20 N_{f}}{9}\left(L_{f}-1\right)\right] g^{\prime 2}+G_{Y, 1}^{2}+12 \lambda_{h}\right\}\right. \\
& \left.+\frac{\mu_{m}^{2}}{4} \tilde{J}_{\Phi}-2 \mu_{m}^{2}\left[\tilde{J}_{\Phi A}^{(1)}+\tilde{J}_{\Phi A}^{(2)}\right]\right),
\end{aligned}
$$

where

$$
G_{Y, 1}^{2} \equiv \frac{2}{3} \operatorname{tr}\left[15 h^{(e)} h^{(e) \dagger}+17 h^{(u)} h^{(u) \dagger}+5 h^{(d)} h^{(d) \dagger}\right] .
$$

Finally, the $h_{3}^{\prime \prime}$ coupling is obtained from the correlator with one $A_{0}^{a}$ and $B_{0}$ external leg,

$$
\begin{aligned}
h_{3}^{\prime \prime}=\frac{g^{\prime} g T}{2}\left\{1+\frac{1}{(4 \pi)^{2}}[\right. & -g^{2}+\frac{1}{3} g^{\prime 2}+L_{b}\left(\frac{43}{12} g^{2}-\frac{1}{12} g^{\prime 2}\right)-N_{f}\left(L_{f}-1\right)\left(\frac{2}{3} g^{2}+\frac{10}{9} g^{\prime 2}\right) \\
& \left.\left.+4 \lambda_{h}+G_{Y, 2}^{2}\right]+\mu_{m}^{2}\left(\frac{1}{4} \tilde{J}_{\Phi}-2 \tilde{J}_{\Phi A B}\right)\right\},
\end{aligned}
$$

where we have defined

$$
\begin{aligned}
\tilde{J}_{\Phi A B} & \equiv-\frac{1}{8} \tilde{J}_{1 / 2}^{4 b}+\frac{1}{2} \tilde{J}_{1 / 3,1,0}^{4 b}, \\
G_{Y, 2}^{2} & \equiv 2 \operatorname{tr}\left[h^{(e)} h^{(e) \dagger}+h^{(u)} h^{(u) \dagger}-h^{(d)} h^{(d) \dagger}\right] .
\end{aligned}
$$

Note that this coupling has a different sign compared to that of ref. [13] due to our different convention for the covariant derivative of the $B_{\mu}$-field.

The combinations of massive master integrals that enter the above expressions for $h_{3}$, $h_{3}^{\prime}$ and $h_{3}^{\prime \prime}$ can be further simplified, as was the case for the $g_{3}$ and $g_{3}^{\prime}$ couplings. A short manipulation shows that

$$
\begin{aligned}
\frac{1}{4} \tilde{J}_{\Phi}-2\left[\tilde{J}_{\Phi A}^{(1)}+\tilde{J}_{\Phi A}^{(2)}\right] & =H\left(\mu_{\sigma}\right), \\
\frac{1}{4} \tilde{J}_{\Phi}-2 \tilde{J}_{\Phi A B} & =H\left(\mu_{\sigma}\right)-\frac{1}{16 \pi^{2} \mu_{\sigma}^{2}},
\end{aligned}
$$

where the integral $H\left(\mu_{\sigma}\right)$ is defined by eq. (B.39).

Finally, the coupling $\delta_{3}$ is obtained from the correlator with two external $C_{0}^{\alpha}$ legs, eq. (3.20)

$$
\delta_{3}=-\frac{2 g_{s}^{2} G_{Y, 3}^{2} T}{(4 \pi)^{2}}
$$

where we defined

$$
G_{Y, 3}^{2} \equiv \operatorname{tr}\left[h^{(u)} h^{(u) \dagger}+h^{(d)} h^{(d) \dagger}\right]
$$

The scalar couplings $\boldsymbol{\mu}_{\boldsymbol{h}, \mathbf{3}}, \boldsymbol{\lambda}_{\boldsymbol{h}, \mathbf{3}}$. The mass parameter of the Higgs doublet is assumed to be heavy, and one-loop corrections contribute to it at the same order. At this order, $g^{2}$, wavefunction renormalization of the Higgs field does not play a role and the squared 
mass parameter in the three-dimensional theory can be extracted directly from eq. (3.32), to which we add the counterterm contributions $\delta \mu_{h}^{2}+\delta \mu_{1} \mu_{m} /\left(2 \mu_{\sigma}^{2}\right)$, the result being

$$
\begin{aligned}
\mu_{h, 3}^{2}= & \mu_{h}^{2}(\Lambda)+\frac{\mu_{m}(\Lambda) \mu_{1}(\Lambda)}{2 \mu_{\sigma}^{2}(\Lambda)}-T^{2}\left(\frac{3}{16} g^{2}+\frac{1}{16} g^{\prime 2}+\frac{\lambda_{h}}{2}+\frac{1}{12} \sum_{i} h_{i}^{2}\right) \\
& +\frac{1}{64 \pi^{2}}\left(2 \mu_{\sigma}^{2} \lambda_{m}-2 \mu_{3} \mu_{m}+\mu_{m}^{2}\right)\left[1+\log \left(\frac{\Lambda^{2}}{\mu_{\sigma}^{2}}\right)\right]+\frac{T^{2}}{16} \frac{\mu_{m}^{2}}{\mu_{\sigma}^{2}} \\
& +J_{1}\left(\mu_{\sigma}\right)\left(-\frac{\lambda_{m}}{2}+\frac{\mu_{3} \mu_{m}}{2 \mu_{\sigma}^{2}}-\frac{\mu_{m}^{2}}{4 \mu_{\sigma}^{2}}\right) .
\end{aligned}
$$

The scalar self-coupling $\lambda_{h, 3}$ can be obtained in a similar fashion from eq. (3.36). This results in a lengthy expressions that is displayed in full in the overview of the matching relations below.

\subsubsection{Collected matching relations at one-loop order}

For the reader's convenience, we collect here all one-loop results for the parameters of the effective theory for the heavy scale. The mass parameters are given to order $g^{2}$, while the couplings are given to order $g^{4}$.

$$
\begin{aligned}
m_{D}^{2}= & g^{2} T^{2}\left(\frac{5}{6}+\frac{N_{f}}{3}\right) \\
m_{D}^{\prime 2}= & g^{\prime 2} T^{2}\left(\frac{1}{6}+\frac{5 N_{f}}{9}\right) \\
m_{D}^{\prime \prime 2}= & g_{s}^{2} T^{2}\left(1+\frac{N_{f}}{6}\right) \\
g_{3}^{2}= & g^{2}(\Lambda) T\left[1+\frac{g^{2}}{(4 \pi)^{2}}\left(\frac{43}{6} L_{b}+\frac{2}{3}-\frac{4 N_{f}}{3} L_{f}\right)\right] \\
g_{3}^{\prime 2}= & g^{\prime 2}(\Lambda) T\left[1+\frac{g^{\prime 2}}{(4 \pi)^{2}}\left(-\frac{1}{6} L_{b}-\frac{20 N_{f}}{9} L_{f}\right)\right] \\
\lambda_{3}= & T \frac{g^{4}}{16 \pi^{2}} \frac{17-4 N_{f}}{3}, \\
\lambda_{3}^{\prime}= & T \frac{g^{\prime 4}}{16 \pi^{2}}\left(\frac{1}{3}-\frac{380}{81} N_{f}\right) \\
\lambda_{3}^{\prime \prime}= & T \frac{g^{2} g^{\prime 2}}{16 \pi^{2}}\left(2-\frac{8}{3} N_{f}\right) \\
h_{3}= & \frac{g^{2}(\Lambda) T}{4}\left(1+\frac{1}{(4 \pi)^{2}}\left\{\left[\frac{43}{6} L_{b}+\frac{17}{2}-\frac{4 N_{f}}{3}\left(L_{f}-1\right)\right] g^{2}+\frac{g^{\prime 2}}{2}-2 g_{Y, 1}^{2}+12 \lambda_{h}\right\}\right. \\
& \left.+\mu_{m}^{2} H\left(\mu_{\sigma}\right)\right) \\
h_{3}^{\prime}= & \frac{g^{\prime 2}(\Lambda) T}{4}\left(1+\frac{1}{(4 \pi)^{2}}\left\{\frac{3 g^{2}}{2}+\left[-\frac{1}{6}\left(L_{b}-1\right)-\frac{20 N_{f}}{9}\left(L_{f}-1\right)\right] g^{\prime 2}-G_{Y, 1}^{2}+12 \lambda_{h}\right\}\right. \\
& \left.+\mu_{m}^{2} H\left(\mu_{\sigma}\right)\right)
\end{aligned}
$$




$$
\begin{aligned}
& h_{3}^{\prime \prime}=\frac{g(\Lambda) g^{\prime}(\Lambda) T}{2}\left\{1+\frac{1}{(4 \pi)^{2}}\left[-g^{2}+\frac{1}{3} g^{\prime 2}+L_{b}\left(\frac{43}{12} g^{2}-\frac{1}{12} g^{\prime 2}\right)\right.\right. \\
& \left.\left.-N_{f}\left(L_{f}-1\right)\left(\frac{2}{3} g^{2}+\frac{10}{9} g^{\prime 2}\right)+4 \lambda_{h}+G_{Y, 2}^{2}\right]+\mu_{m}^{2}\left[H\left(\mu_{\sigma}\right)-\frac{1}{16 \pi^{2} \mu_{\sigma}^{2}}\right]\right\} \text {, } \\
& \delta_{3}=-\frac{2 g_{s}^{2} G_{Y, 3}^{2} T}{(4 \pi)^{2}} \\
& \mu_{h, 3}^{2}=\mu_{h}^{2}(\Lambda)+\frac{\mu_{m}(\Lambda) \mu_{1}(\Lambda)}{2 \mu_{\sigma}^{2}(\Lambda)}-T^{2}\left(\frac{3}{16} g^{2}+\frac{1}{16} g^{\prime 2}+\frac{\lambda_{h}}{2}+\frac{1}{12} \sum_{i} h_{i}^{2}\right) \\
& +\frac{1}{64 \pi^{2}}\left(2 \mu_{\sigma}^{2} \lambda_{m}-2 \mu_{3} \mu_{m}+\mu_{m}^{2}\right)\left[1+\log \left(\frac{\Lambda^{2}}{\mu_{\sigma}^{2}}\right)\right] \\
& +\frac{T^{2}}{16} \frac{\mu_{m}^{2}}{\mu_{\sigma}^{2}}+J_{1}\left(\mu_{\sigma}\right)\left(-\frac{\lambda_{m}}{2}+\frac{\mu_{3} \mu_{m}}{2 \mu_{\sigma}^{2}}-\frac{\mu_{m}^{2}}{4 \mu_{\sigma}^{2}}\right) \text {, } \\
& \lambda_{h, 3}=T\left\{\lambda_{h}(\Lambda)-\frac{1}{8} \frac{\mu_{m}^{2}(\Lambda)}{\mu_{\sigma}^{2}(\Lambda)}-\frac{1}{4} \frac{\mu_{m}^{2} \mu_{3} \mu_{1}(\Lambda)}{\mu_{\sigma}^{6}}+\frac{1}{2} \frac{\mu_{m} \lambda_{m} \mu_{1}(\Lambda)}{\mu_{\sigma}^{4}}\right. \\
& +\frac{1}{16 \pi^{2}} \frac{1}{16}\left\{6 g^{4}+4 g^{2} g^{\prime 2}+2 g^{\prime 4}+L_{f}\left(16 \sum_{i} h_{i}^{4}-32 \lambda_{h} g_{Y, 1}^{2}\right)\right. \\
& \left.-3 L_{b}\left[3 g^{4}+g^{\prime 4}+2 g^{\prime 2}\left(g^{2}-4 \lambda_{h}\right)+8 \lambda_{h}\left(-3 g^{2}+8 \lambda_{h}\right)\right]\right\} \\
& -\frac{1}{16 \pi^{2}} \frac{\mu_{m}^{2}}{\mu_{\sigma}^{2}} \frac{1}{2}\left[L_{b}\left(\frac{9}{8} g^{2}+\frac{3}{8} g^{\prime 2}\right)-\frac{1}{2} L_{f} g_{Y, 1}^{2}\right]+\left(\frac{1}{2} \lambda_{h} \mu_{m}^{2}-\frac{1}{16} \frac{\mu_{m}^{4}}{\mu_{\sigma}^{2}}\right)\left[H\left(\mu_{\sigma}\right)+\frac{1}{16 \pi^{2} \mu_{\sigma}^{2}}\right] \\
& -\frac{1}{2 \mu_{\sigma}^{4}}\left[\frac { 1 } { 1 2 8 \pi ^ { 2 } } \left(\mu_{\sigma}^{2}\left[\mu_{m}^{2}\left(24 \lambda_{h}+6 \lambda_{\sigma}-12 \lambda_{m}\right)+8 \mu_{3} \lambda_{m} \mu_{m}\right]+\mu_{m}^{2}\left(-4 \mu_{3}^{2}+4 \mu_{3} \mu_{m}-3 \mu_{m}^{2}\right)\right.\right. \\
& \left.+L_{b} \mu_{m}^{2}\left(3 \mu_{m}^{2}-48 \mu_{\sigma}^{2} \lambda_{h}\right)+\log \left(\frac{\Lambda^{2}}{\mu_{\sigma}^{2}}\right)\left\{\mu_{m}^{2}\left[24 \mu_{\sigma}^{2} \lambda_{h}+2 \mu_{\sigma}^{2}\left(3 \lambda_{\sigma}-4 \lambda_{m}\right)-2 \mu_{m}^{2}\right]+4 \mu_{\sigma}^{4} \lambda_{m}^{2}\right\}\right) \\
& +T^{2} \mu_{m}^{2}\left(\frac{\lambda_{h}}{4}-\frac{\mu_{m}^{2}}{32 \mu_{\sigma}^{2}}+\frac{\mu_{3} \mu_{m}}{12 \mu_{\sigma}^{2}}-\frac{\lambda_{m}}{4}\right) \\
& +J_{1}\left(\mu_{\sigma}\right)\left[\mu_{m}^{2}\left(-3 \lambda_{h}+\frac{3 \mu_{m}^{2}}{8 \mu_{\sigma}^{2}}-\frac{\mu_{3} \mu_{m}}{2 \mu_{\sigma}^{2}}+\frac{\mu_{3}^{2}}{2 \mu_{\sigma}^{2}}-\frac{3 \lambda_{\sigma}}{4}+\frac{3 \lambda_{m}}{2}\right)-\mu_{3} \lambda_{m} \mu_{m}\right] \\
& \left.\left.+J_{2}\left(\mu_{\sigma}\right)\left[\mu_{m}^{2}\left(\frac{\mu_{m}^{2}}{8}-\frac{\mu_{3} \mu_{m}}{2}+\frac{\mu_{3}^{2}}{2}+\frac{\mu_{\sigma}^{2} \lambda_{m}}{2}\right)-\mu_{\sigma}^{2} \mu_{3} \lambda_{m} \mu_{m}+\mu_{\sigma}^{4} \frac{\lambda_{m}^{2}}{2}\right]\right]\right\} .
\end{aligned}
$$

In these expressions, we have used the following notation introduced earlier in this section:

$$
\begin{aligned}
L_{b} & \equiv 2 \ln \left(\frac{\Lambda}{T}\right)-2[\ln (4 \pi)-\gamma], \\
L_{f} & \equiv L_{b}+4 \ln 2, \\
g_{Y, 1}^{2} & \equiv \operatorname{tr}\left[h^{(e)} h^{(e) \dagger}+3 h^{(u)} h^{(u) \dagger}+3 h^{(d)} h^{(d) \dagger}\right] \approx 3 g_{Y}^{2}, \\
G_{Y, 1}^{2} & \equiv \frac{2}{3} \operatorname{tr}\left[15 h^{(e)} h^{(e) \dagger}+17 h^{(u)} h^{(u) \dagger}+5 h^{(d)} h^{(d) \dagger}\right] \approx \frac{34}{3} g_{Y}^{2}, \\
G_{Y, 2}^{2} & \equiv 2 \operatorname{tr}\left[h^{(e)} h^{(e) \dagger}+h^{(u)} h^{(u) \dagger}-h^{(d)} h^{(d) \dagger}\right] \approx 2 g_{Y}^{2}, \\
G_{Y, 3}^{2} & \equiv \operatorname{tr}\left[h^{(u)} h^{(u) \dagger}+h^{(d)} h^{(d) \dagger}\right] \approx g_{Y}^{2} .
\end{aligned}
$$


The given approximate values apply when only the top quark Yukawa coupling $g_{Y}$ is nonzero. We also have $\sum_{i} h_{i}^{n} \approx 3 g_{Y}^{n}$, since here the sum runs over the spectrum of Gram matrices of the Yukawa couplings, including the three-fold degeneracy due to different colors,

$$
h_{i} \in \operatorname{spectrum}\left(\sqrt{h^{(e)} h^{(e) \dagger}}, \sqrt{h^{(u)} h^{(u) \dagger}}, \sqrt{h^{(d)} h^{(d) \dagger}}\right) .
$$

The massive master integrals $J_{1}(m), J_{2}(m)$ and $H(m)$ are defined in appendix B.

\subsection{Integration over the heavy scale}

The effective theory for the heavy scale is already identical to the SM case, only differing by the contributions of the new scalar $\sigma$ to the effective couplings. The next step, in which heavy degrees of freedom are integrated out to leave an effective theory for the light scale alone, therefore goes through without any modification. In particular, the matching conditions that relate the couplings of the effective theories for the heavy and light scales can be taken from ref. [13]. However, in addition we include here the leading contribution of the temporal gluons to the scalar mass parameter in the last term in eq. (3.107). We list these matching conditions here for the reader's convenience,

$$
\begin{aligned}
\bar{g}_{3}^{2} & =g_{3}^{2}\left(1-\frac{g_{3}^{2}}{24 \pi m_{D}}\right), \\
\bar{g}_{3}^{\prime 2} & =g_{3}^{\prime 2} \\
\bar{\mu}_{h, 3}^{2} & =\mu_{h, 3}^{2}+\frac{1}{4 \pi}\left(3 h_{3} m_{D}+h_{3}^{\prime} m_{D}^{\prime}+8 \delta_{3} m_{D}^{\prime \prime}\right), \\
\bar{\lambda}_{h, 3} & =\lambda_{h, 3}-\frac{1}{8 \pi}\left(\frac{3 h_{3}^{2}}{m_{D}}+\frac{h_{3}^{\prime 2}}{m_{D}^{\prime}}+\frac{h_{3}^{\prime \prime 2}}{m_{D}+m_{D}^{\prime}}\right) .
\end{aligned}
$$

\subsection{Relations to physical parameters}

In this section, we have derived expressions for the parameters of the three-dimensional effective theories of the SSM in terms of the running $\overline{\mathrm{MS}}$ parameters of the original fourdimensional theory. The ultimate aim is to translate the behavior of the effective theory into physical insights concerning, amongst other things, the order of the electroweak phase transition in the full theory. To do this, however, we need to express the $\overline{\mathrm{MS}}$ parameters in terms of measurable quantities such as pole masses and the Fermi constant. In this article, we have worked only up to one-loop order in the scalar mass parameters $\left(\sim g^{2}\right)$ so it suffices to perform this translation at tree level. However, if our matching results are eventually generalised to two-loop order, providing $g^{4}$ accuracy, then one would need the $\overline{\mathrm{MS}}$ parameters to be related to the physical ones at the same $g^{4}$ order, requiring a one-loop renormalization of the theory [13]. This rather tedious exercise is left to a forthcoming paper.

For the gauge couplings, we use the Standard Model results of ref. [13],

$$
\begin{aligned}
g^{2} & =g_{0}^{2}, \\
g^{\prime 2} & =\frac{g_{0}^{2}}{m_{W}^{2}}\left(m_{Z}^{2}-m_{W}^{2}\right),
\end{aligned}
$$


where we have denoted $g_{0}^{2} \equiv 4 \sqrt{2} G_{f} m_{W}^{2}$, with $G_{f}$ being the Fermi constant. By inverting the mass eigenvalues (cf. section 3.1.3) for both of the physical scalars, the $\mathrm{W}$ boson and the top quark, one can on the other hand obtain the desired tree-level relations for the corresponding parameters. An important simplification can be achieved by fixing the parameter $\mu_{1}$ as given in eq. (2.15) such that singlet Vacuum Expectation Value vanishes, $\rho=0$, while the doublet VEV is the same as in the SM, i.e. $\nu=\mu_{h} / \sqrt{\lambda_{h}}$. As a result, we obtain

$$
\begin{aligned}
& \mu_{h}^{2}=\frac{1}{4}\left[m_{-}^{2}+m_{+}^{2} \pm \frac{\left.\sqrt{g_{0}^{4} m_{W}^{4}\left(m_{-}^{2}-m_{+}^{2}\right)^{2}-4 g_{0}^{2} \mu_{m}^{2} m_{W}^{6}}\right]}{g_{0}^{2} m_{W}^{2}}\right], \\
& \lambda_{h}=\frac{g_{0}^{2} m_{W}^{2}\left(m_{-}^{2}+m_{+}^{2}\right) \pm \sqrt{g_{0}^{4} m_{W}^{4}\left(m_{-}^{2}-m_{+}^{2}\right)^{2}-4 g_{0}^{2} \mu_{m}^{2} m_{W}^{6}}}{16 m_{W}^{4}}, \\
& \mu_{\sigma}^{2}=\frac{1}{2}\left[m_{-}^{2}+m_{+}^{2}-\frac{4 \lambda_{m} m_{W}^{4} \pm \sqrt{g_{0}^{4} m_{W}^{4}\left(m_{-}^{2}-m_{+}^{2}\right)^{2}-4 g_{0}^{2} \mu_{m}^{2} m_{W}^{6}}}{g_{0}^{2} m_{W}^{2}}\right],
\end{aligned}
$$

where one must consistently take the same sign in all three equations. Identifying the Higgs mass with $m_{-}$requires us to take the positive sign for each.

It is easy to see that in the decoupling limit where the portal couplings vanish, the above relations reduce to those of the SM. Here, it would have been possible to eliminate one of the portal couplings in favor of the mixing angle of the two physical scalars, but for practical reasons we have kept both portal couplings as input parameters. Furthermore, the Yukawa coupling obeys the relation

$$
g_{Y}^{2}=\frac{g_{0}^{2}}{2} \frac{m_{t}^{2}}{m_{W}^{2}},
$$

which is same as in the SM.

To obtain the $\overline{\mathrm{MS}}$ and effective theory parameters as functions of the renormalization scale, the above relations are used as initial conditions at the scale $\Lambda=m_{Z}$. We emphasize that fixing $\mu_{1}$ in terms of $\mu_{m}$ and the doublet VEV at the initial scale - such that $\rho=0$ there - does not make this parameter vanish, in general. However, by solving $\rho$ and $\nu$ in terms of the coupling constants by requiring that they minimize the tree-level scalar potential, and allowing these expressions to run with the renormalization scale, the changes in the VEVs remain numerically small.

\section{Discussion}

In the present work, we have performed a high-temperature dimensional reduction of the Standard Model augmented by a singlet scalar field coupled in the most general way to the Higgs field. For our purposes, the singlet is treated as a superheavy degree of freedom and integrated out of the theory altogether; the only light fields remaining in the 3D theory correspond to the Higgs, $\mathrm{SU}(2)$ and $\mathrm{U}(1)$ zero modes. As a consequence, the presence of the singlet in the 4D theory appears through the enlarged RG-system of couplings (eq. (3.40)(3.50)); through the multiple occurrences of the non-SM couplings $\mu_{\sigma}^{2}, \mu_{m}, \mu_{1}, \mu_{3}, \lambda_{m}$ 


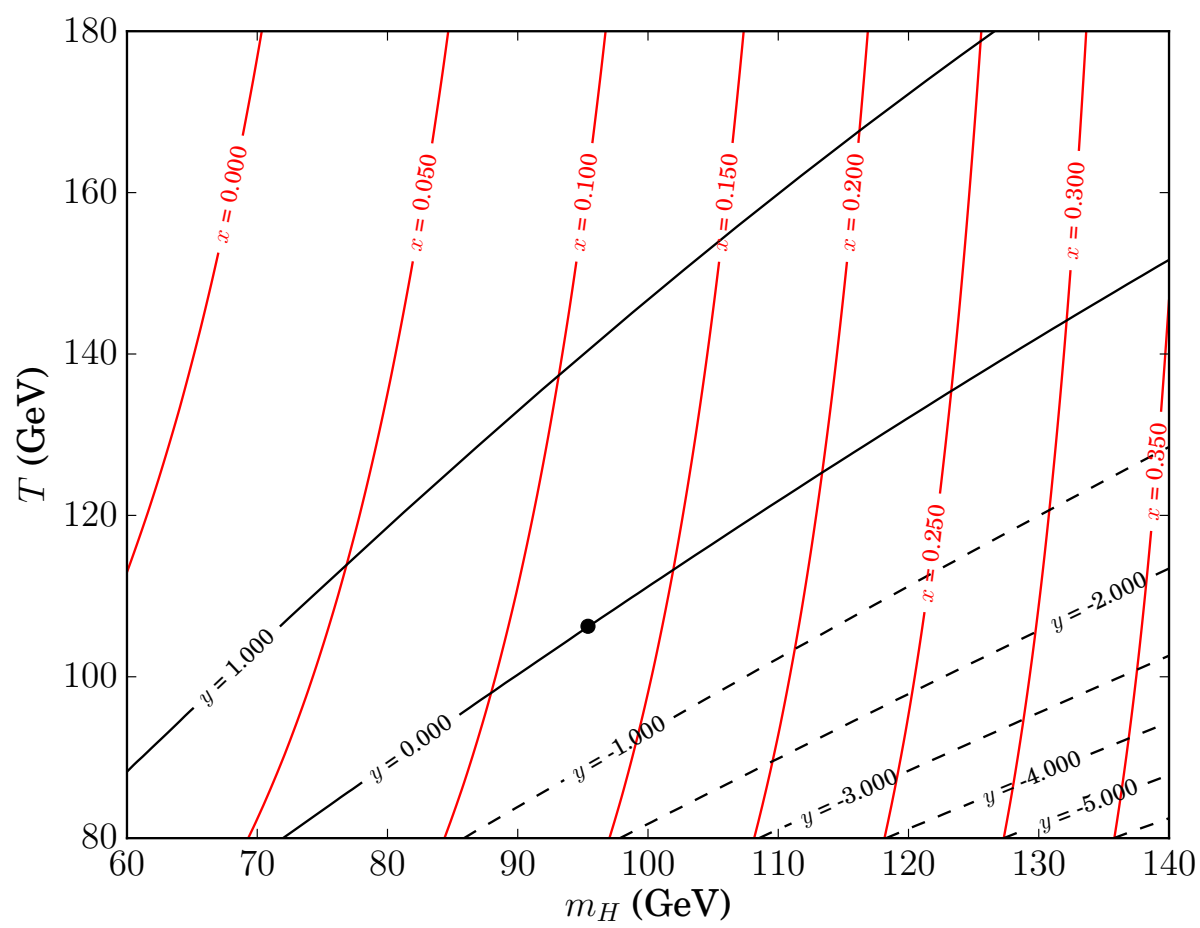

Figure 1. The $m_{H}-T$ plane in the Standard Model. Overlaid, curves of constant $x$ and $y$ as defined in the main text. The black dot denotes the critical point in the 3D theory. This can be compared with figure 8 of ref. [13], but note that we have chosen a different set of approximations in carrying out the dimensional reduction, as explained in the main text.

and $\lambda_{\sigma}$ in the matching relations in section 3.3.3 (and after integrating out the heavy scale in section 3.4); and through the matching to physical parameters as described in section 3.5. The SM limit (taking $\lambda_{m}$ and $\mu_{m}$ to zero) stands out clearly in the expressions of section 3.3.3, and we note that the singlet addition is a highly non-trivial generalisation of these expressions.

We match to the exact same $3 \mathrm{D}$ theory as in the seminal papers $[14,15]$, where the nonperturbative lattice simulations are phrased in terms of the dimensionless combinations

$$
\frac{\bar{g}_{3}^{2}}{T}, \quad x=\frac{\bar{\lambda}_{3}}{\bar{g}_{3}^{2}}, \quad y=\frac{\bar{m}_{3}^{2}}{\bar{g}_{3}^{4}} .
$$

It turns out that $\bar{g}_{3}^{2}$ varies very little for the parameter range considered, and one is left with finding the position of the phase transition in $x$ - $y$-space. This computation involves only the $3 \mathrm{D}$ theory, and the result applies to any $4 \mathrm{D}$ theory that is matched to it. As one might expect, the phase transition happens near $y=0$, where the mass parameter $\bar{m}_{3}^{2}$ changes sign. The central result of $[15,16]$ is that there is a line $0<x<x_{c}, y \simeq 0$, where the phase transition is first order. This line ends at a critical point $x_{c} \simeq 0.125$, beyond which the transition is a crossover.

In figure 1 we show the Higgs mass-temperature $\left(T-m_{H}\right)$ plane for the Standard Model. Overlaid are curves of constant $x$ and $y$ as defined by the matching relations. We have marked the point $\left(x_{c}, y=0\right)$ with a black dot, and we see that it corresponds to a value 


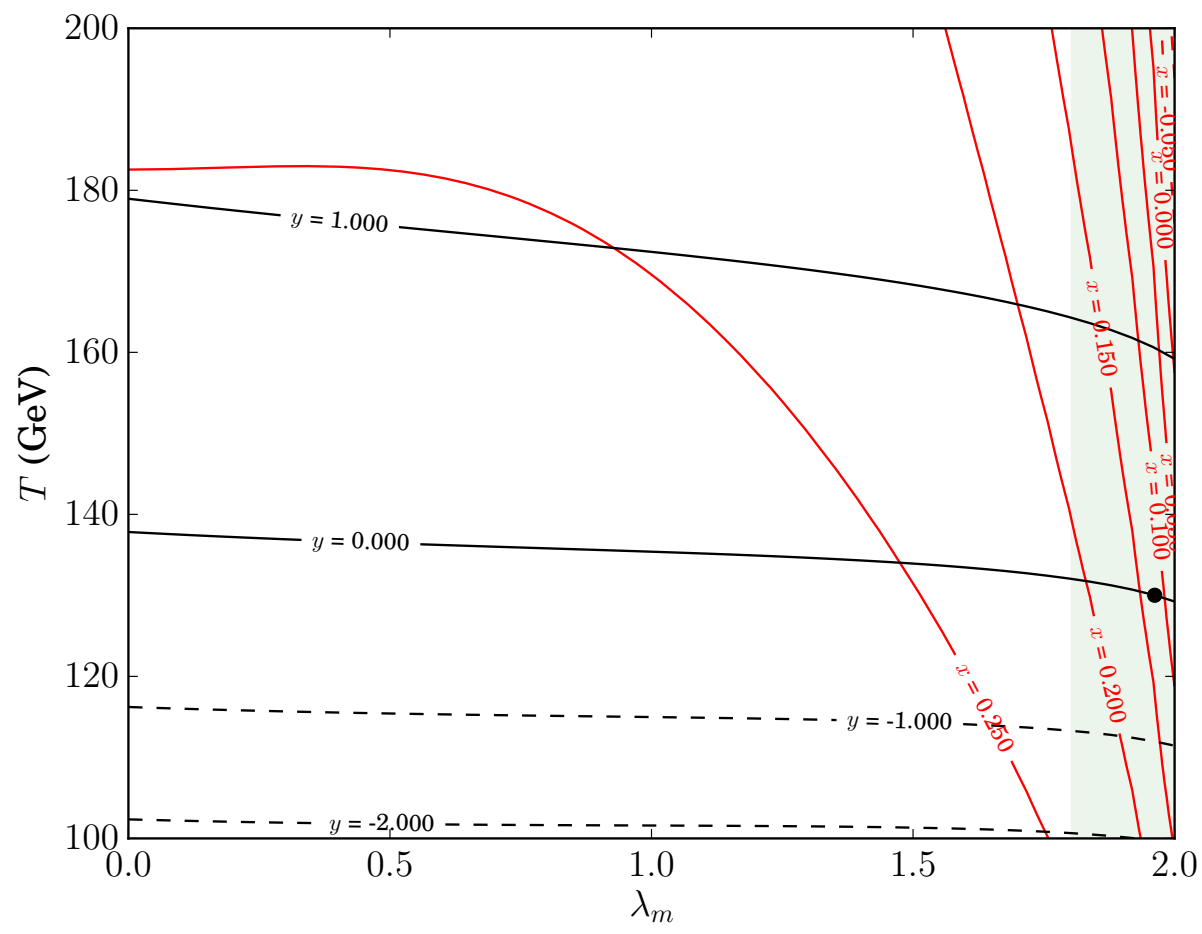

Figure 2. The $\lambda_{m}$-T plane in the $Z_{2}$-symmetric singlet-extended Standard Model, when $m_{\sigma}=$ $250 \mathrm{GeV}$ and $\lambda_{\sigma}=1 / 4$. Overlaid, curves of constant $x$ and $y$ as defined in the main text. The black dot denotes the critical point in the $3 \mathrm{D}$ theory. In the shaded region the computation is unreliable due to $\mu_{h}>\mu_{\sigma}$, which violates our assumption about scale hierarchy for mass parameters.

of $m_{H}$ much below the measured value of $\simeq 125 \mathrm{GeV}$. The figure 1 can be compared with figure 8 of ref. [13]. The difference between these two is due to a different set of approximations in carrying out the dimensional reduction: while we have included order $g^{\prime 4}$ effect of $\mathrm{U}(1)_{Y}$ gauge field and the effect of temporal gluons, we have not included two-loop contributions to the mass parameter of Higgs, nor the one-loop relations between $\overline{\mathrm{MS}}$ parameters and physical quantities. Major difference comes from the omission of two-loop contributions.

The familiar conclusion is that in the Standard Model, the transition is a crossover. One may approximately recover the whole first order range by following the $y=0$ line from the black dot towards $x=0$. Nonperturbative simulations give a slight deviation from $y=0$, but the conclusion is the same. We see that the physical point has $x_{S M} \simeq 0.25$ at $y=0$. This is one way of quantizing "how far" the Standard Model is from the first order range.

The object of this work is to investigate whether adding a singlet allows for a first order transition while insisting that $m_{H}=125 \mathrm{GeV}$. The difference that the singlet makes is that because the matching relations have changed, for a given set of $4 \mathrm{D}$ parameters in the 5-dimensional SM-singlet parameter space, varying the temperature over the electroweak transition results in a different trajectory in $\left\{\bar{g}_{3}^{2}, \bar{g}_{3}^{\prime 2}, \bar{\mu}_{h, 3}^{2}, \bar{\lambda}_{h, 3}^{2}\right\}$-space, and, in turn, in $x-y$ space. The task is therefore to identify these trajectories and perform similar multicanonical simulations $[14,15]$. For a complete scan of the singlet model, this is a challenge, but not impossible. Fortunately, comprehensive scans already exist employing perturbation theory 


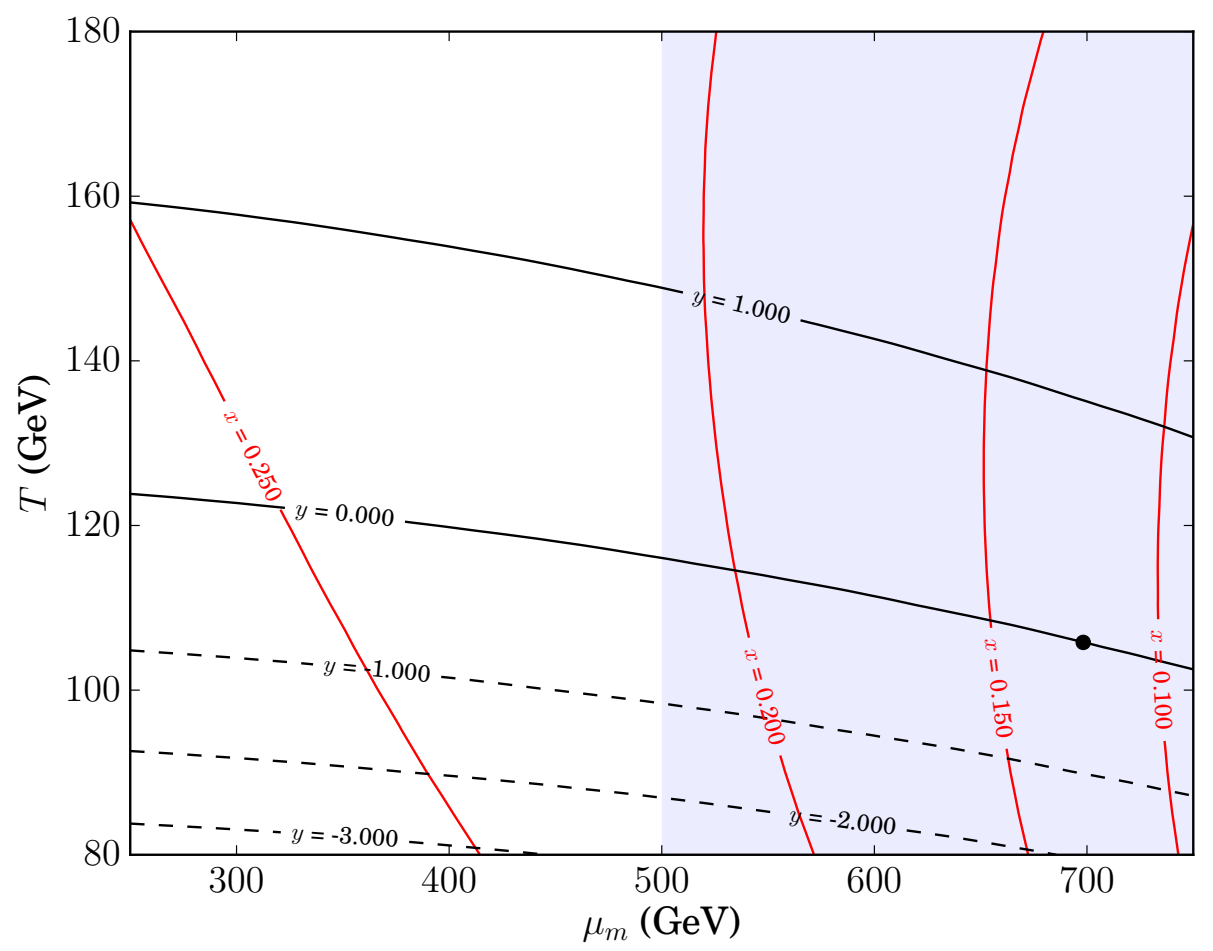

Figure 3. The $\mu_{m}-T$ plane in the singlet-extended Standard Model, when $m_{\sigma}=500 \mathrm{GeV}, \lambda_{m}=0$, $\mu_{3}=0$ and $\lambda_{\sigma}=1 / 4$. Overlaid, curves of constant $x$ and $y$ as defined in the main text. The black dot denotes the critical point in the $3 \mathrm{D}$ theory. In the shaded region the computation is unreliable due to $\mu_{m}>\mu_{\sigma}$, which violates our scaling assumptions.

computations of the $4 \mathrm{D}$ effective potential which, together with experimental constraints, may be used to guide non-perturbative searches [42-51]. A detailed numerical investigation of this theory with the matchings presented here is underway [52].

For the present, we show in figure 2 a pencil in the 5 -dimensional parameter space, where we impose $Z_{2}$-symmetry $\left(\mu_{3}=\mu_{m}=0\right)$. Guided by existing perturbative results, we choose $\lambda_{\sigma}=1 / 4$ and $m_{\sigma}=250 \mathrm{GeV}$, and scan over the remaining parameter, the quartic portal coupling $\lambda_{m}$. Overlaid are again curves of constant $x$ and $y$, and we have again placed a black dot at the point $\left(x_{c}, y=0\right)$ corresponding to the critical point. Following the $y=0$ line towards smaller $x$ gives the first order range.

We see that the critical point requires $\lambda_{m} \simeq 2$. This is rather large, which jeopardizes the validity of our perturbative matching relations. Another, more serious issue is that the whole first order line is located in a region where $\mu_{h}>\mu_{\sigma}$, explicitly violating one of the assumptions of we made about how the mass parameters scale, namely that the $\sigma$ field is superheavy. For more details, see section 2.1.2. Hence although the new matching relations numerically allow us to approach the first order region, we cannot go closer than $x=0.2$ and still trust our computation. That large couplings are necessary in the $Z_{2}$-symmetric case is also true perturbatively (see for instance [51]).

However $Z_{2}$-symmetry need not be imposed, and in figure 3 we show the case where $\lambda_{m}=0, \lambda_{\sigma}=1 / 4, \mu_{3}=0$ and where we have chosen $m_{\sigma}=500 \mathrm{GeV}$, while varying the cubic 
portal coupling $\mu_{m}$. We see that the critical point is within the scope of the matching relations, but it turns out that another of our scaling assumptions $\mu_{m}<\mu_{\sigma}$ is not fulfilled. The closest we can go seems to again be $x \simeq 0.2$, and we have been unable to find a parameter set that obeys all the assumptions of section 2.1.2 while providing a first order transition. We have however not systematically scanned the full parameter space in the present paper.

We conclude that, within the limits of our current approximation, we cannot argue that adding a singlet provides a first order electroweak phase transition. We do believe that at least in the non- $Z_{2}$-symmetric case, effort would be well spent on improving on this approximation with the view of confirming the picture in figure 3 . In the case of superheavy $\sigma$, our current approximation can be improved by adding two-loop contributions to the mass parameters in the dimensional reduction step. Another improvement would be to give the relations between $\overline{\mathrm{MS}}$ parameters and physical quantities to one-loop accuracy. With these improvements, one would obtain full $g^{4}$ accuracy, analogous to ref. [13] for the Standard Model. This work is already underway.

There are many other generalisations of our results that deserve further consideration. One could treat the singlet as a heavy rather than a superheavy field, which would still allow us to integrate it out, and the theory would still reduce to the same 3D theory. Another possibility is to include higher order operators (dimension 6 and above) in the 3D effective theory. It is also possible to treat the singlet as a light field, permitting the (expectation value of the) singlet to play an active role in the phase transition, with different values in the high- and low-temperature phases. This is in contrast to the present case, where the super-heavy singlet only acts as an additional spectator degree of freedom, impinging on the Higgs effective potential through modified effective couplings. If the singlet was light, a 3D singlet-Higgs potential would come into play. Then the numerics would involve a whole new 3D theory, with some additional work required for a consistent lattice implementation.

Other 4D theories also deserve investigation. A strong candidate for future study is the Two-Higgs Doublet Model. This consists of two equivalent Higgs fields coupled to each other and to gauge fields, and with one (Type I) or both (Type II) of the Higgs fields coupled to fermions. Work on this is already underway. Several attempts have been made on computing the strength of the $2 \mathrm{HDM}$ phase transition perturbatively [56-60], most recently in [61]. However, the full $2 \mathrm{HDM}$ parameters space is 10-dimensional, posing an even bigger numerical challenge than the singlet model addressed here. On the other hand, the $2 \mathrm{HDM}$ readily allows for the inclusion of $\mathrm{CP}$-violation, which the singlet model itself does not. ${ }^{7}$ From the point of view of baryogenesis, this is appealing, whereas it has no relevance for sourcing observable gravitational waves.

\section{Acknowledgments}

TT has been supported by the Vilho, Yrjö and Kalle Väisälä Foundation and AV was supported by the Academy of Finland grant nos. 1273545 and 1303622. DJW was supported by the People Programme (Marie Skłodowska-Curie actions) of the European Union Seventh

\footnotetext{
${ }^{7}$ One may remedy this by adding CP-violating higher dimensional operators.
} 
Framework Programme (FP7/2007-2013) under grant agreement number PIEF-GA-2013629425. This research was supported by the Munich Institute for Astro- and Particle Physics (MIAPP) of the DFG cluster of excellence "Origin and Structure of the Universe". The authors would like to thank Keijo Kajantie, Mikko Laine and Kari Rummukainen for enlightening discussions.

\section{A Feynman rules in the unbroken phase}

In this appendix we list the Feynman rules valid in the high-temperature phase where the expectation value of the Higgs field vanishes. Note that our list is not complete in that we leave out the gluon sector, which is not needed for dimensional reduction at the order considered here.

\section{Projectors to specific polarization states.}

$$
\begin{aligned}
\text { Transverse projector: } & \mathcal{P}_{T}(K)_{\mu \nu} \equiv \delta_{\mu \nu}-\frac{K_{\mu} K_{\nu}}{K^{2}} \\
\text { Chiral projectors: } & \mathcal{P}_{R} \equiv \frac{1}{2}\left(1+\gamma_{5}\right), \quad \mathcal{P}_{L} \equiv \frac{1}{2}\left(1-\gamma_{5}\right)
\end{aligned}
$$

\section{A.1 Propagators in the Landau gauge}

$$
\begin{aligned}
& \mathrm{SU}(2)_{L} \text { gauge bosons: } \sim \sim \sim \delta^{a b} \frac{\mathcal{P}_{T}(K)_{\mu \nu}}{K^{2}} \\
& \mathrm{U}(1)_{Y} \text { gauge boson: } \mathbf{W M M M}=\frac{\mathcal{P}_{T}(K)_{\mu \nu}}{K^{2}} \\
& \mathrm{SU}(3)_{c} \text { gauge bosons: } \text { ணூ }=\delta^{\alpha \beta} \frac{\mathcal{P}_{T}(K)_{\mu \nu}}{K^{2}} \\
& \mathrm{SU}(2)_{L} \text { ghosts: } \cdots \cdots \cdots=\delta^{a b} \frac{1}{K^{2}} \\
& \text { fermions: }=\mathcal{P}_{L / R} \frac{\mathrm{i}}{\mathbb{K}_{K}} \quad \text { (left/right-handed) } \\
& \text { Higgs doublet: } \quad-----=\delta^{i j} \frac{1}{K^{2}} \\
& \text { neutral scalar: } \quad=\frac{1}{K^{2}+\mu_{\sigma}^{2}}
\end{aligned}
$$

In the case of heavy $\sigma$, its propagator is to be expanded in powers of $\mu_{\sigma}^{2}$.

\section{A.2 Interaction vertices}

For oriented lines, momentum is understood to flow in the given direction. For unoriented lines, momentum flows into the interaction vertex. 


\section{Gauge self-interactions.}

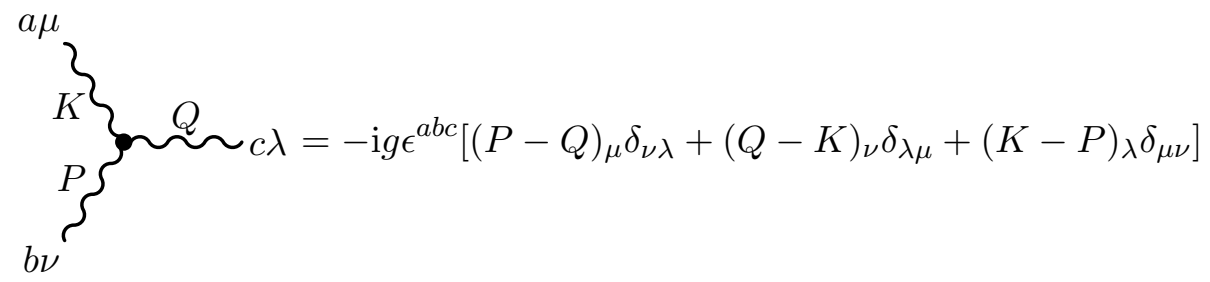

When $c \lambda$ is an external line with $Q=0$, this vertex reduces in the Landau gauge to

$$
2 \mathrm{i} g \epsilon^{a b c} P_{\lambda} \delta_{\mu \nu}=-2 \mathrm{i} g \epsilon^{a b c} K_{\lambda} \delta_{\mu \nu} .
$$
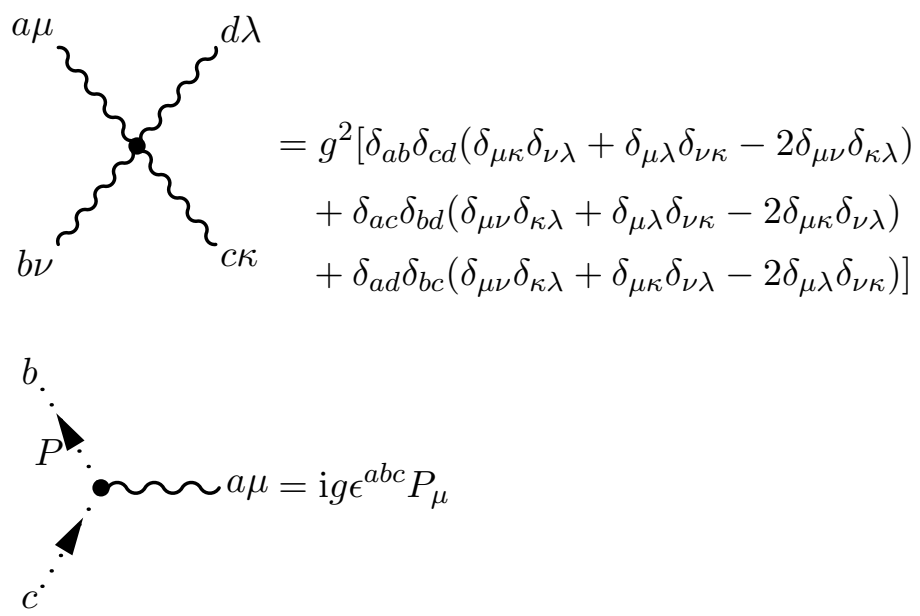

\section{Gauge-matter interactions.}
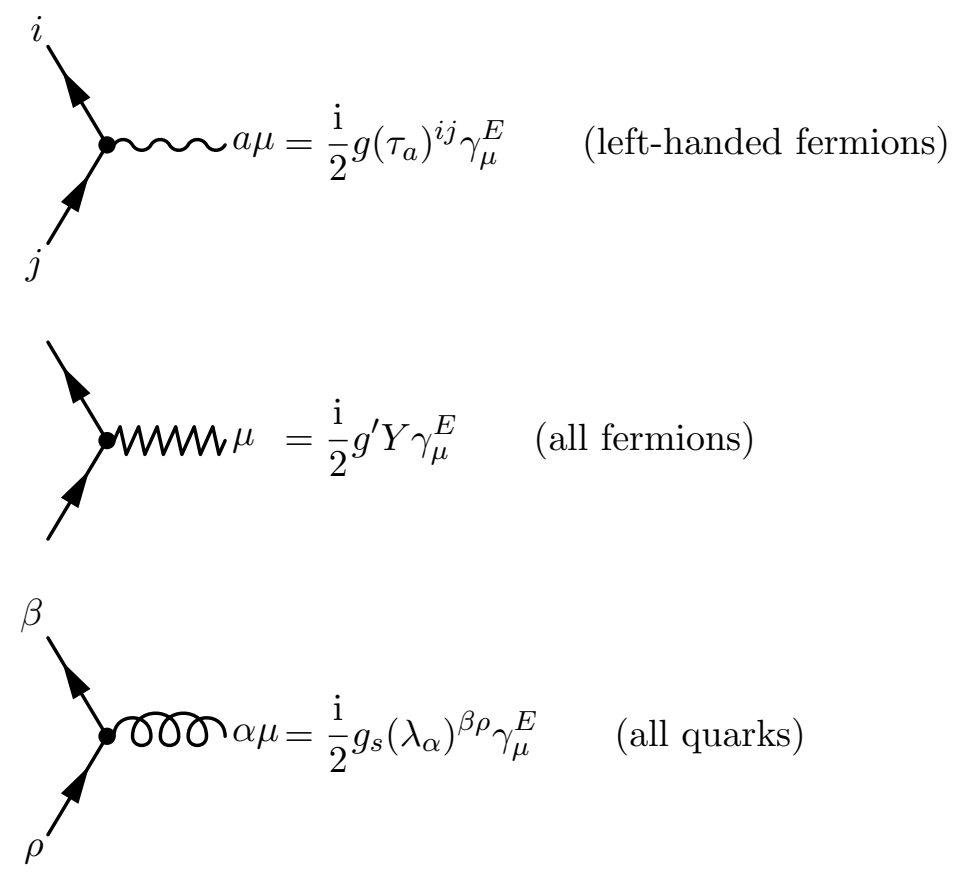

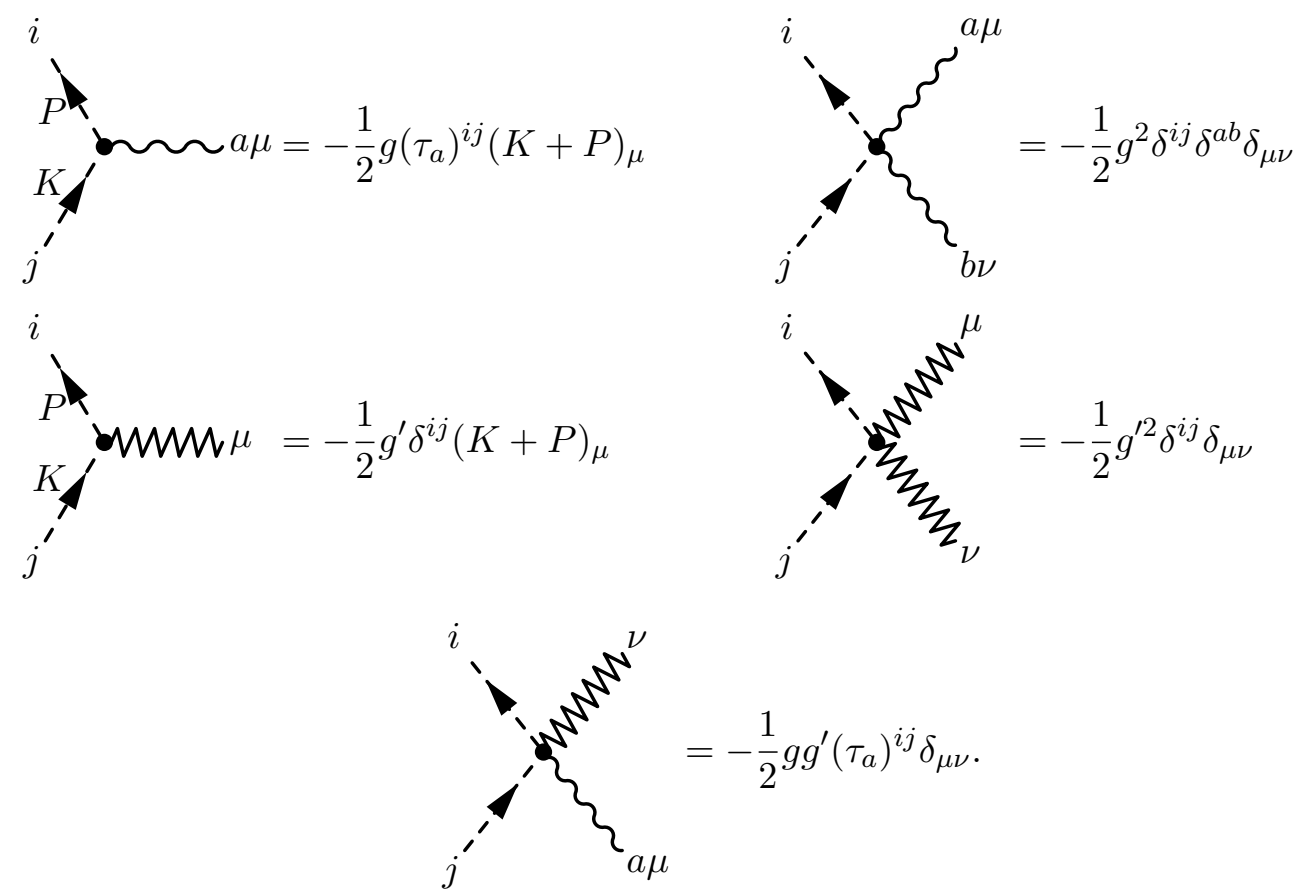

Scalar self-interactions.

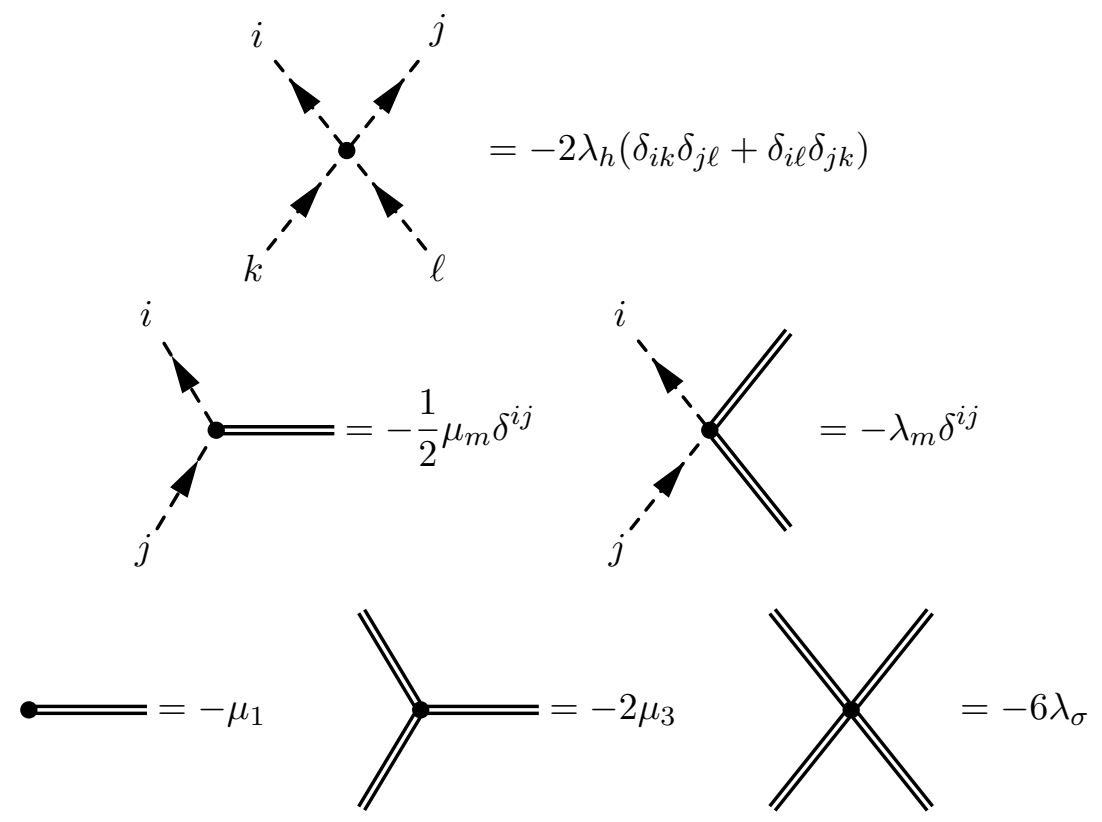

Yukawa interactions. The family indices are indicated explicitly.
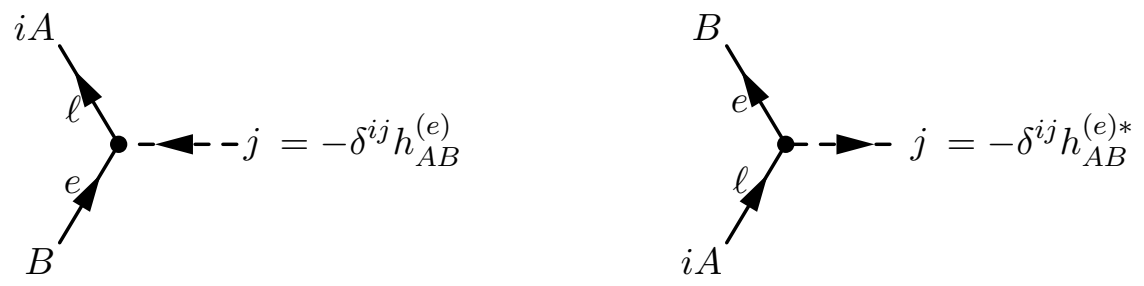

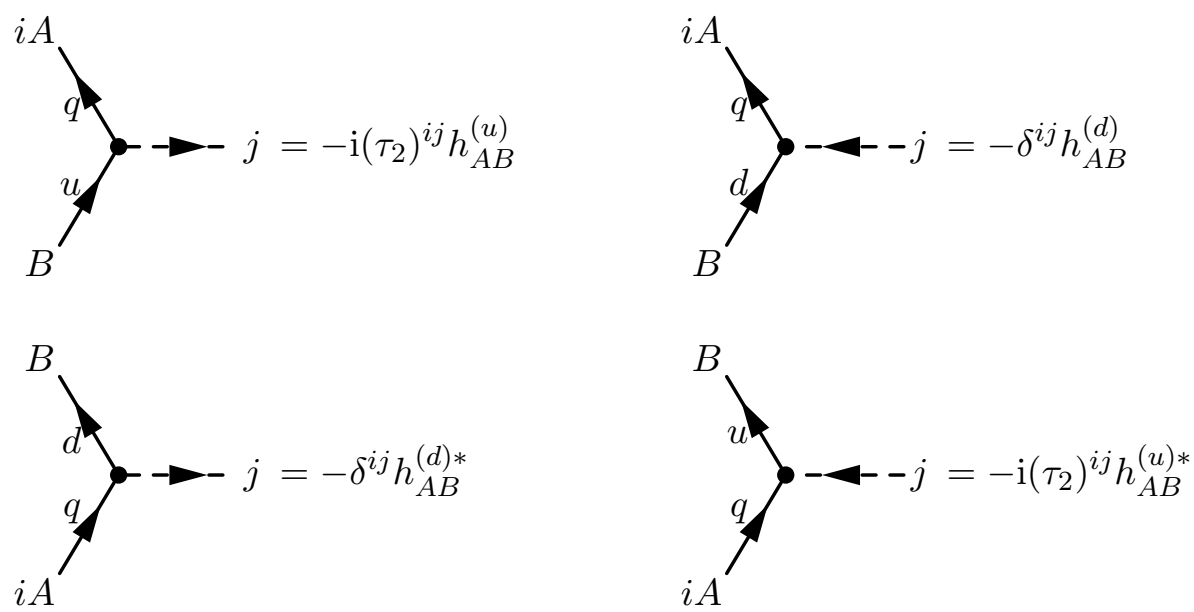

\section{B Integrals for the dimensional reduction step}

For spatial momentum integration, we use the shorthand notation

$$
\int_{p} \equiv\left(\frac{e^{\gamma} \Lambda^{2}}{4 \pi}\right)^{\epsilon} \int \frac{\mathrm{d}^{d} \boldsymbol{p}}{(2 \pi)^{d}}
$$

where $d \equiv 3-2 \epsilon$. The Euclidean four-momentum is denoted as $P=\left(\omega_{n}, \boldsymbol{p}\right)$ for bosons, where $\omega_{n} \equiv 2 n \pi T$, and as $P=\left(\nu_{n}, \boldsymbol{p}\right)$ for fermions, where $\nu_{n} \equiv(2 n+1) \pi T$. For the combined Matsubara sum and spatial momentum integration, we use the following shorthand:

$$
\text { bosons: } \begin{aligned}
\mathcal{f}_{P} & \equiv T \sum_{\omega_{n}} \int_{p}, \\
& \mathcal{f}_{P}^{\prime} \equiv T \sum_{\omega_{n} \neq 0} \int_{p} \quad \text { (sum over nonzero modes), }
\end{aligned}
$$

fermions: $\quad \sum_{\{P\}} \equiv T \sum_{\nu_{n}} \int_{p}$.

\section{B.1 Massless bosonic sum-integrals}

$$
\begin{aligned}
& I_{\alpha, \beta, \delta}^{4 b} \equiv \sum_{P}^{\prime} \frac{\left(P_{0}^{2}\right)^{\beta}\left(\boldsymbol{p}^{2}\right)^{\delta}}{\left(P^{2}\right)^{\alpha}}= \frac{\left(e^{\gamma} \Lambda^{2}\right)^{\epsilon}}{8 \pi^{2}} \frac{\Gamma\left(\alpha-\frac{d}{2}-\delta\right) \Gamma\left(\frac{d}{2}+\delta\right) \zeta(2 \alpha-2 \beta-2 \delta-d)}{\Gamma\left(\frac{1}{2}\right) \Gamma(\alpha) \Gamma\left(\frac{d}{2}\right)} \\
& \times(2 \pi T)^{1+d-2 \alpha+2 \beta+2 \delta}, \\
& I_{\alpha, \beta}^{4 b} \equiv I_{\alpha, \beta, 0}^{4 b}=\sum_{P}^{\prime} \frac{\left(P_{0}^{2}\right)^{\beta}}{\left(P^{2}\right)^{\alpha}}= \frac{\left(e^{\gamma} \Lambda^{2}\right)^{\epsilon}}{8 \pi^{2}} \frac{\Gamma\left(\alpha-\frac{d}{2}\right) \zeta(2 \alpha-2 \beta-d)}{\Gamma\left(\frac{1}{2}\right) \Gamma(\alpha)}(2 \pi T)^{1+d-2 \alpha+2 \beta}, \\
& I_{\alpha}^{4 b} \equiv I_{\alpha, 0}^{4 b}=\sum_{P}^{\prime} \frac{1}{\left(P^{2}\right)^{\alpha}}=\frac{\left(e^{\gamma} \Lambda^{2}\right)^{\epsilon}}{8 \pi^{2}} \frac{\Gamma\left(\alpha-\frac{d}{2}\right) \zeta(2 \alpha-d)}{\Gamma\left(\frac{1}{2}\right) \Gamma(\alpha)}(2 \pi T)^{1+d-2 \alpha,} \\
& I_{1}^{4 b}=\sum_{P}^{\prime} \frac{1}{P^{2}}=\frac{T^{2}}{12}\left(\frac{\Lambda}{4 \pi T}\right)^{2 \epsilon}\left\{1+2\left[\log 2 \pi+\gamma-\frac{\zeta^{\prime}(2)}{\zeta(2)}\right] \epsilon+\mathcal{O}\left(\epsilon^{2}\right)\right\}, \\
& I_{2}^{4 b}=\sum_{P}^{\prime} \frac{1}{\left(P^{2}\right)^{2}}=\frac{1}{16 \pi^{2}}\left(\frac{\Lambda}{4 \pi T}\right)^{2 \epsilon}\left[\frac{1}{\epsilon}+2 \gamma+\mathcal{O}(\epsilon)\right] .
\end{aligned}
$$


Useful recursive relations among the sum-integrals:

$$
\begin{aligned}
I_{\alpha+1, \beta+1}^{4 b} & =\left(1-\frac{d}{2 \alpha}\right) I_{\alpha, \beta}^{4 b}, \\
I_{\alpha+1, \beta, \delta+1}^{4 b} & =\frac{\frac{d}{2}+\delta}{\alpha} I_{\alpha, \beta, \delta}^{4 b}, \\
I_{\alpha, \beta-1, \delta+1}^{4 b} & =-\frac{\frac{d}{2}+\delta}{1+\frac{d}{2}-\alpha+\delta} I_{\alpha, \beta, \delta}^{4 b} .
\end{aligned}
$$

Occasionally, we need analogous sum-integrals including the zero Matsubara mode; these are denoted by a tilde, e.g. $\tilde{I}_{\alpha, \beta, \delta}^{4 b}$. Explicit expressions for these sum-integrals are not needed, we merely note that they do not satisfy the above recursive relations.

\section{B.2 Massless fermionic sum-integrals}

$$
\begin{aligned}
I_{\alpha, \beta, \delta}^{4 f} \equiv \sum_{\{P\}} \frac{\left(P_{0}^{2}\right)^{\beta}\left(\boldsymbol{p}^{2}\right)^{\delta}}{\left(P^{2}\right)^{\alpha}}=\left(2^{2 \alpha-2 \beta-2 \delta-d}-1\right) I_{\alpha, \beta, \delta}^{4 b} \\
I_{\alpha, \beta}^{4 f} \equiv I_{\alpha, \beta, 0}^{4 f}=\sum_{\{P\}} \frac{\left(P_{0}^{2}\right)^{\beta}}{\left(P^{2}\right)^{\alpha}} \\
I_{\alpha}^{4 f} \equiv I_{\alpha, 0}^{4 f}=\sum_{\{P\}} \frac{1}{\left(P^{2}\right)^{\alpha}} \\
I_{1}^{4 f}=\sum_{\{P\}} \frac{1}{P^{2}}=-\frac{T^{2}}{24}\left(\frac{\Lambda}{4 \pi T}\right)^{2 \epsilon}\left\{1+2\left[\log \pi+\gamma-\frac{\zeta^{\prime}(2)}{\zeta(2)}\right] \epsilon+\mathcal{O}\left(\epsilon^{2}\right)\right\} \\
I_{2}^{4 f}=\sum_{\{P\}} \frac{1}{\left(P^{2}\right)^{2}}=\frac{1}{16 \pi^{2}}\left(\frac{\Lambda}{4 \pi T}\right)^{2 \epsilon}\left[\frac{1}{\epsilon}+2 \gamma+4 \log 2+\mathcal{O}(\epsilon)\right]
\end{aligned}
$$

Due to the first of the above relations, the fermionic sum-integrals satisfy the same recursive identities as their bosonic counterparts.

\section{B.3 Massive sum-integrals}

$$
\begin{aligned}
\tilde{K}^{4 b}(m) & \equiv \frac{1}{2} \sum_{P} \log \left(P^{2}+m^{2}\right), \\
\tilde{K}^{4 f}(m) & \equiv \frac{1}{2} \sum_{\{P\}} \log \left(P^{2}+m^{2}\right), \\
\tilde{J}_{\kappa / \alpha, \beta, \delta}^{4 b}(m) & \equiv \sum_{P} \frac{\left(P_{0}^{2}\right)^{\beta}\left(\boldsymbol{p}^{2}\right)^{\delta}}{\left(P^{2}\right)^{\alpha}\left(P^{2}+m^{2}\right)^{\kappa}}, \\
\tilde{J}_{\kappa / \alpha}^{4 b}(m) & \equiv \tilde{J}_{\kappa / \alpha, 0,0}^{4 b}=\sum_{P} \frac{1}{\left(P^{2}\right)^{\alpha}\left(P^{2}+m^{2}\right)^{\kappa}}, \\
\tilde{J}_{\kappa}^{4 b}(m) & \equiv \tilde{J}_{\kappa / 0}^{4 b}=\sum_{P} \frac{1}{\left(P^{2}+m^{2}\right)^{\kappa}},
\end{aligned}
$$

and likewise for the version without the zero mode, $J_{\kappa / \alpha, \beta, \delta}^{4 b}(m)$. For $\beta>0$, the two integrals - with and without the zero mode - coincide. The two-index integrals satisfy the recursive relation

$$
\tilde{J}_{\kappa / \alpha}^{4 b}(m)=\tilde{J}_{\kappa-1 / \alpha+1}^{4 b}(m)-m^{2} \tilde{J}_{\kappa / \alpha+1}^{4 b}(m) .
$$


It is straightforward to verify that the following relations hold:

$$
\begin{aligned}
\tilde{J}_{1 / 1}^{4 b}(m) & =\frac{1}{m^{2}}\left[I_{1}^{4 b}-\tilde{J}_{1}^{4 b}(m)\right] \\
\tilde{J}_{1 / 2}^{4 b}(m) & =\frac{1}{m^{2}} I_{2}^{4 b}-\frac{1}{m^{4}}\left[I_{1}^{4 b}-\tilde{J}_{1}^{4 b}(m)\right] \\
\tilde{J}_{2 / 1}^{4 b}(m) & =-\frac{1}{m^{2}} \tilde{J}_{2}^{4 b}(m)+\frac{1}{m^{4}}\left[I_{1}^{4 b}-\tilde{J}_{1}^{4 b}(m)\right], \\
\tilde{J}_{2 / 2}^{4 b}(m) & =\frac{1}{m^{4}}\left[I_{2}^{4 b}+\tilde{J}_{2}^{4 b}(m)\right]-\frac{2}{m^{6}}\left[I_{1}^{4 b}-\tilde{J}_{1}^{4 b}(m)\right], \\
\tilde{J}_{1 / 3,0,1}^{4 b}(m) & =\frac{1}{m^{2}} I_{3,0,1}^{4 b}-\frac{1}{m^{4}} I_{2,0,1}^{4 b}+\frac{1}{m^{6}} I_{1,0,1}^{4 b}-\frac{1}{m^{6}} \tilde{J}_{1 / 0,0,1}^{4 b}(m), \\
\tilde{J}_{1 / 3,1,0}^{4 b}(m) & =\tilde{J}_{1 / 2}^{4 b}(m)-\tilde{J}_{1 / 3,0,1}^{4 b}(m), \\
\tilde{J}_{3 / 1,0,1}^{4 b}(m) & =-\frac{1}{m^{2}} \tilde{J}_{3 / 0,0,1}^{4 b}(m)-\frac{1}{m^{4}} \tilde{J}_{2 / 0,0,1}^{4 b}(m)-\frac{1}{m^{6}} \tilde{J}_{1 / 0,0,1}^{4 b}(m)+\frac{1}{m^{6}} I_{1,0,1}^{4 b} \cdot
\end{aligned}
$$

Furthermore

$$
\begin{aligned}
\tilde{J}_{1}^{4 b}(m) & =I_{1}^{4}(m)+J_{1}(m), \\
\tilde{J}_{2}^{4 b}(m) & =I_{2}^{4}(m)+J_{2}(m), \\
\tilde{J}_{3 / 0,0,1}^{4 b}(m) & =\frac{3-2 \epsilon}{4-2 \epsilon}\left[I_{2}^{4}(m)-m^{2} I_{3}^{4}(m)\right]+J_{3,0,1}(m), \\
\tilde{J}_{2 / 0,0,1}^{4 b}(m) & =\frac{3-2 \epsilon}{4-2 \epsilon}\left[I_{1}^{4}(m)-m^{2} I_{2}^{4}(m)\right]+J_{2,0,1}(m), \\
\tilde{J}_{1 / 0,0,1}^{4 b}(m) & =-\frac{3-2 \epsilon}{4-2 \epsilon} m^{2} I_{1}^{4}(m)+J_{1,0,1}(m),
\end{aligned}
$$

where we have defined

$$
I_{\alpha}^{4}(m) \equiv\left(\frac{e^{\gamma} \Lambda^{2}}{4 \pi}\right)^{\epsilon} \int \frac{\mathrm{d}^{n} p}{(2 \pi)^{n}} \frac{1}{\left(p^{2}+m^{2}\right)^{\alpha}}=\left(\frac{e^{\gamma} \Lambda^{2}}{4 \pi}\right)^{\epsilon} \frac{\left(m^{2}\right)^{\frac{n}{2}-\alpha}}{(4 \pi)^{\frac{n}{2}}} \frac{\Gamma\left(\alpha-\frac{n}{2}\right)}{\Gamma(\alpha)},
$$

where $n=4-2 \epsilon$ and

$$
\begin{array}{rlrl}
J_{1}(m) & \equiv \int \frac{\mathrm{d}^{3} \boldsymbol{p}}{(2 \pi)^{3}} \frac{n_{B}\left(E_{p}\right)}{E_{p}}, & J_{1,0,1}(m) & \equiv \int \frac{\mathrm{d}^{3} \boldsymbol{p}}{(2 \pi)^{3}} \frac{p^{2} n_{B}\left(E_{p}\right)}{E_{p}}, \\
J_{2}(m) \equiv \int \frac{\mathrm{d}^{3} \boldsymbol{p}}{(2 \pi)^{3}} \frac{n_{B}\left(E_{p}\right)}{2 p^{2} E_{p}}, & J_{2,0,1}(m) \equiv \int \frac{\mathrm{d}^{3} \boldsymbol{p}}{(2 \pi)^{3}} \frac{3 n_{B}\left(E_{p}\right)}{2 E_{p}} \\
J_{3,0,1}(m) & \equiv \int \frac{\mathrm{d}^{3} \boldsymbol{p}}{(2 \pi)^{3}} \frac{3 n_{B}\left(E_{p}\right)}{8 p^{2} E_{p}}
\end{array}
$$

where $n_{B}$ is the Bose-Einstein distribution function and $E_{p} \equiv \sqrt{\boldsymbol{p}^{2}+m^{2}}$. These integrals satisfy the simple relations

$$
J_{2,0,1}(m)=\frac{3}{2} J_{1}(m), \quad J_{3,0,1}(m)=\frac{3}{4} J_{2}(m) .
$$

Thus, the only master integrals needed are actually just $J_{1}(m), J_{2}(m)$ and $J_{1,0,1}(m)$. The summary of the results in the case of superheavy $\sigma$ only features explicitly $J_{1}(m), J_{2}(m)$ and the following particular combination of the three integrals:

$$
H(m) \equiv-\frac{3}{32 \pi^{2} m^{2}}-\frac{1}{m^{4}}\left[\frac{T^{2}}{12}+J_{1}(m)\right]+\frac{1}{m^{6}}\left[\frac{2 \pi^{2} T^{4}}{45}-\frac{4 J_{1,0,1}(m)}{3}\right] .
$$




\section{Detailed results for the SM contributions to dimensional reduction}

Below we provide a list of all one-loop diagrams in the SM that arise in the four-dimensional theory, expressed in terms of the master sum-integrals introduced in appendix B. New contributions from the neutral scalar are discussed in section 3.1. All the diagrams listed below are given without the zero mode contribution, which at the one-loop level trivially drops in the matching to the three-dimensional effective theory. Many of the diagrams have already been calculated in ref. [13], but some of the contributions of the $\mathrm{U}(1)_{Y}$ sector included here are new. The indicated values of the diagrams already include combinatorial factors due to permutations of external lines.

\section{C.1 Self-energy diagrams}

These are needed for the calculation of wave-function renormalization and of the Debye masses of the gauge bosons. The diagrams with a single quartic vertex only contribute to the latter. The wavefunction renormalization factors can be read off from the parts that are quadratic in momentum, as detailed in section 3.2.

\section{$\mathrm{SU}(2)_{L}$ gauge boson self-energy.}

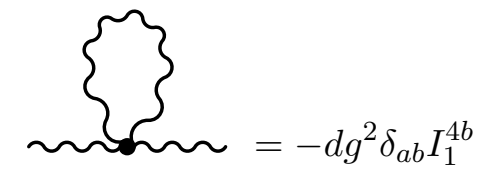

for $\mu=\nu=0$,

$=g^{2}(1-2 d) \delta_{a b} \delta_{r s} I_{1}^{4 b}$

for $\mu=r, \nu=s$,

$$
\begin{aligned}
& \text { for } \mu=\nu=0, \\
& =2 d g^{2} \delta_{a b} \delta_{r s} I_{1}^{4 b}+g^{2} \delta_{a b}\left[\frac{1}{6}(31-2 d) \delta_{r s} P^{2}+\frac{1}{3}(d-17) P_{r} P_{s}\right] I_{2}^{4 b} \\
& \text { for } \mu=r, \nu=s,
\end{aligned}
$$

$$
\begin{aligned}
& \begin{array}{l}
\sim \quad \because \text { on }=g^{2} \delta_{a b}\left[(d-2) I_{1}^{4 b}+\frac{1}{6}(4-d) P^{2} I_{2}^{4 b}\right] \\
\text { for } \mu=\nu=0,
\end{array} \\
& =-g^{2} \delta_{a b} \delta_{r s} I_{1}^{4 b}+\frac{1}{6} g^{2} \delta_{a b}\left(\delta_{r s} P^{2}+2 P_{r} P_{s}\right) I_{2}^{4 b}
\end{aligned}
$$

for $\mu=r, \nu=s$, 
$\sim \sim g^{2}(d-1) \delta_{a b} N_{f}\left(1+N_{c}\right)\left[\left(2^{2-d}-1\right) I_{1}^{4 b}-\frac{1}{6}\left(2^{4-d}-1\right) P^{2} I_{2}^{4 b}\right]$

for $\mu=\nu=0$,

$$
=\frac{1}{3} g^{2}\left(2^{4-d}-1\right) \delta_{a b} N_{f}\left(1+N_{c}\right)\left(P_{r} P_{s}-\delta_{r s} P^{2}\right) I_{2}^{4 b}
$$

for $\mu=r, \nu=s$,

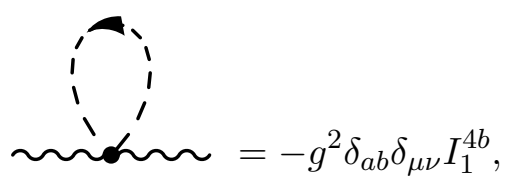

for $\mu=\nu=0$,

$$
=g^{2} \delta_{a b}\left[\delta_{r s} I_{1}^{4 b}+\frac{1}{6}\left(P_{r} P_{s}-\delta_{r s} P^{2}\right) I_{2}^{4 b}\right]
$$

for $\mu=r, \nu=s$.

$\mathrm{U}(1)_{Y}$ gauge boson self-energy.

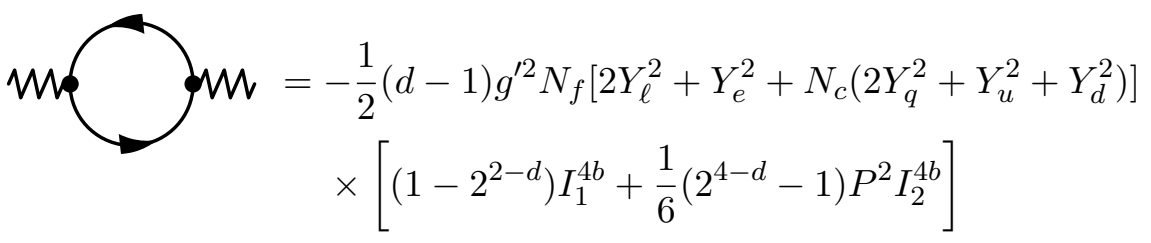

for $\mu=\nu=0$,

$$
\begin{aligned}
= & \frac{1}{6}\left(2^{4-d}-1\right) g^{\prime 2} N_{f}\left[2 Y_{\ell}^{2}+Y_{e}^{2}+N_{c}\left(2 Y_{q}^{2}+Y_{u}^{2}+Y_{d}^{2}\right)\right] \\
& \times\left(P_{r} P_{s}-\delta_{r s} P^{2}\right) I_{2}^{4 b}
\end{aligned}
$$

for $\mu=r, \nu=s$,

$$
\text { Wh }
$$




$$
\begin{aligned}
& \text { for } \mu=\nu=0 \\
& =g^{\prime 2}\left[\delta_{r s} I_{1}^{4 b}+\frac{1}{6}\left(P_{r} P_{s}-\delta_{r s} P^{2}\right) I_{2}^{4 b}\right] \\
& \text { for } \mu=r, \nu=s
\end{aligned}
$$

Higgs doublet self-energy. Only diagrams contributing to wavefunction renormalization are shown here; the mass parameter can be extracted from the effective potential.

$$
\begin{aligned}
& \text { ל } \\
& \sum_{\text {LW }} \\
& \begin{aligned}
- & 2 \delta_{i j} \operatorname{tr}\left[h^{(e)} h^{(e) \dagger}+N_{c} h^{(u)} h^{(u) \dagger}+N_{c} h^{(d)} h^{(d) \dagger}\right] \\
& \times\left[\left(2^{2-d}-1\right) I_{1}^{4 b}-\frac{1}{2}\left(2^{4-d}-1\right) P^{2} I_{2}^{4 b}\right] .
\end{aligned}
\end{aligned}
$$

\section{C.2 Correlators for gauge fields}

The various four-point correlators with two or four gauge field external legs are listed below in the same order as in section 3.1.2.

The $A_{0}^{a} A_{0}^{b} A_{0}^{c} A_{0}^{d}$ correlator.

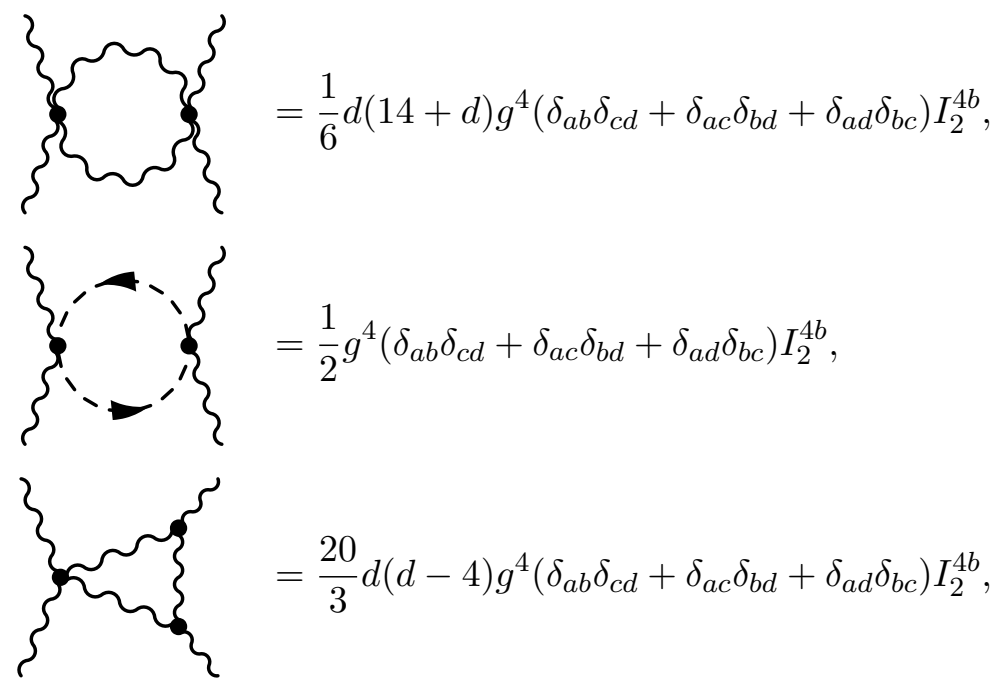




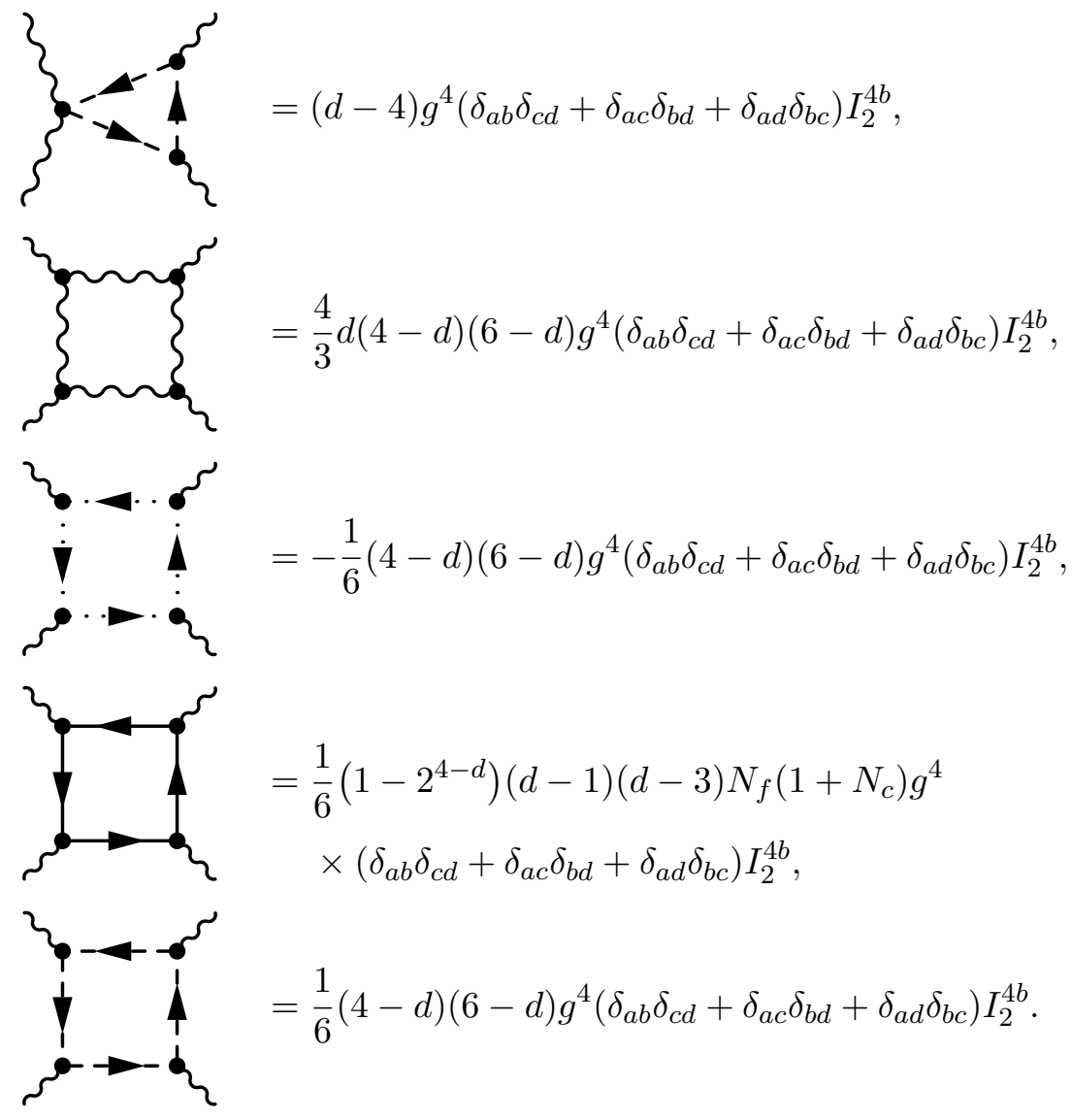

The $B_{0}^{4}$ correlator.

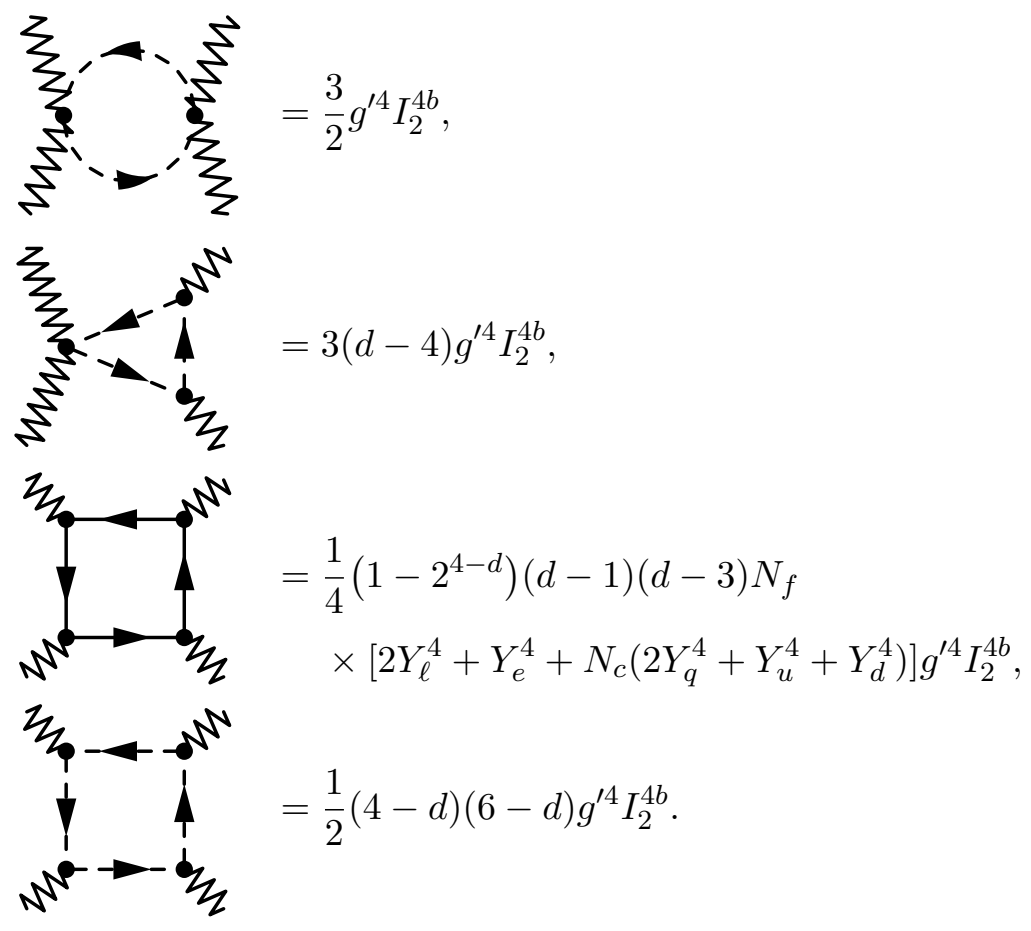


The $A_{0}^{a} A_{0}^{b} B_{0}^{2}$ correlator.

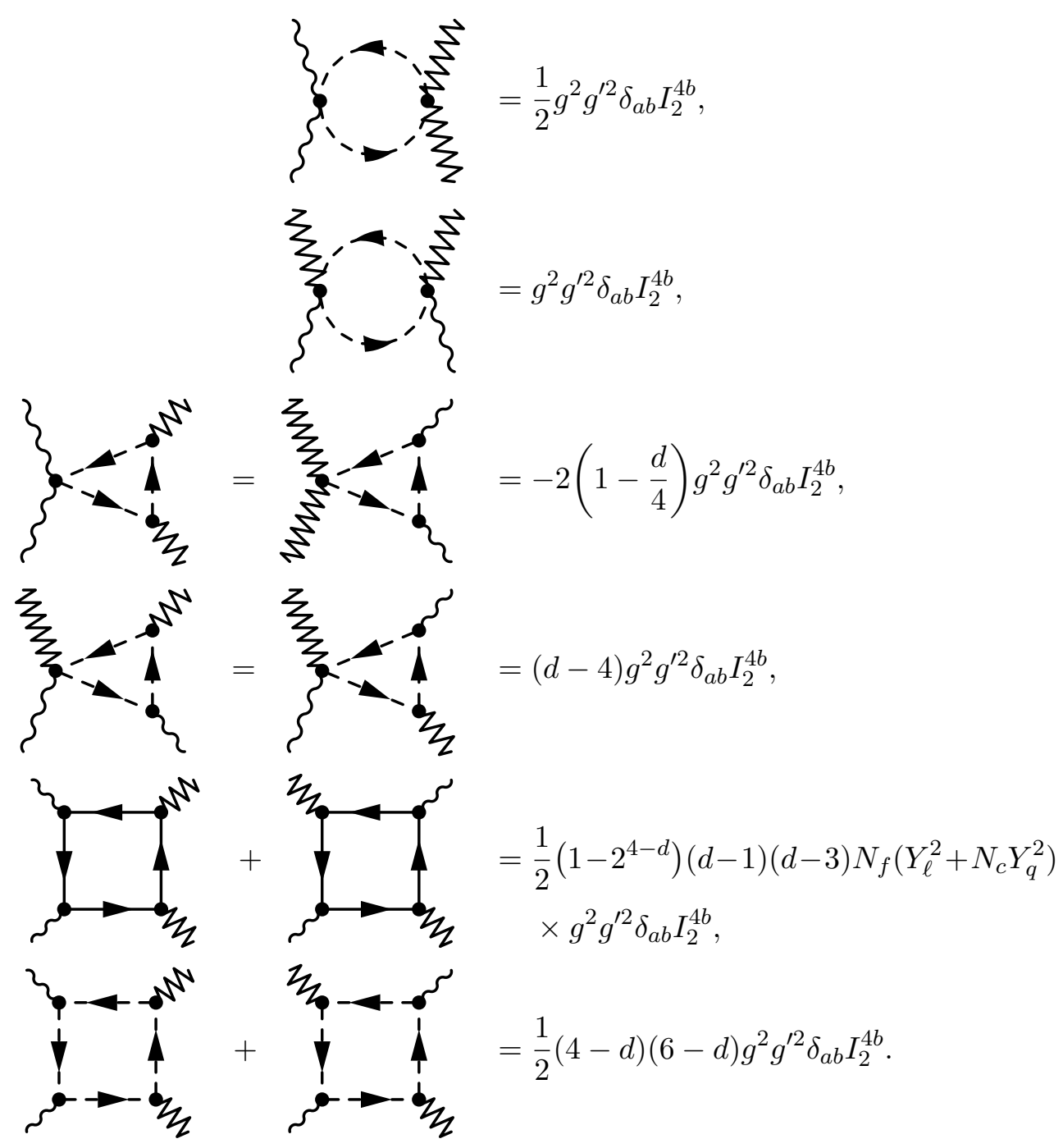

The $\phi^{\dagger i} \phi^{j} A_{\mu}^{a} A_{\nu}^{b}$ correlator.

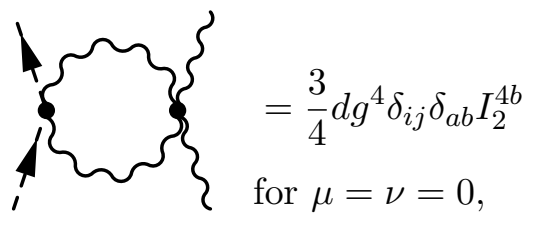

$$
=\left(d-\frac{3}{4}\right) g^{4} \delta_{i j} \delta_{a b} \delta_{r s} I_{2}^{4 b}
$$

for $\mu=r, \nu=s$,

ר. 


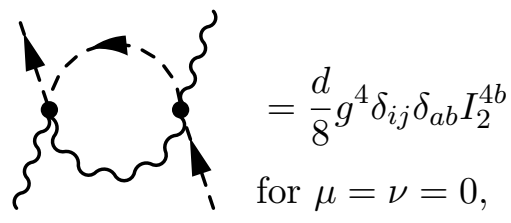

$=\frac{3}{8} g^{4} \delta_{i j} \delta_{a b} \delta_{r s} I_{2}^{4 b}$

for $\mu=r, \nu=s$,

$\left\{\begin{array}{l}=\frac{d}{8} g^{2} g^{\prime 2} \delta_{i j} \delta_{a b} I_{2}^{4 b} \\ \text { for } \mu=\nu=0,\end{array}\right.$

$=\frac{3}{8} g^{2} g^{\prime 2} \delta_{i j} \delta_{a b} \delta_{r s} I_{2}^{4 b}$

for $\mu=r, \nu=s$,

$\left\{\begin{array}{l}\text { for } \mu=\nu=0, \\ =d(d-4) g^{4} \delta_{i j} \delta_{a b} I_{2}^{4 b}\end{array}\right.$

$=-d g^{4} \delta_{i j} \delta_{a b} \delta_{r s} I_{2}^{4 b}$

for $\mu=r, \nu=s$,

$\left\{\begin{array}{l}\text { for } \mu=\nu=0, \\ \boldsymbol{\alpha}\end{array}\right.$

$=-3 \lambda_{h} g^{2} \delta_{i j} \delta_{a b} \delta_{r s} I_{2}^{4 b}$

for $\mu=r, \nu=s$,

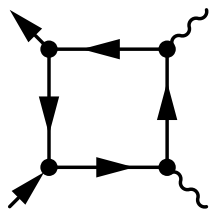

$=\frac{1}{2}\left(2^{4-d}-1\right)(2-d) g^{2} \delta_{i j} \delta_{a b} \operatorname{tr}\left[h^{(e)} h^{(e) \dagger}+N_{c} h^{(d)} h^{(d) \dagger}\right] I_{2}^{4 b}$

for $\mu=\nu=0$,

$=-\frac{1}{2}\left(2^{4-d}-1\right) g^{2} \delta_{i j} \delta_{a b} \delta_{r s} \operatorname{tr}\left[h^{(e)} h^{(e) \dagger}+N_{c} h^{(d)} h^{(d) \dagger}\right] I_{2}^{4 b}$

for $\mu=r, \nu=s$, 


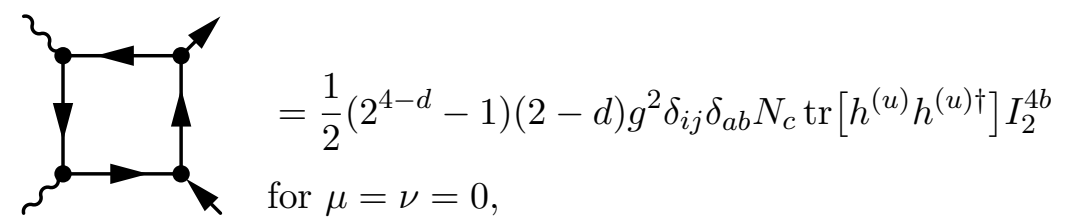

$$
=-\frac{1}{2}\left(2^{4-d}-1\right) g^{2} \delta_{i j} \delta_{a b} \delta_{r s} N_{c} \operatorname{tr}\left[h^{(u)} h^{(u) \dagger}\right] I_{2}^{4 b}
$$

for $\mu=r, \nu=s$.

The $\phi^{\dagger i} \phi^{j} B_{\mu} B_{\nu}$ correlator.
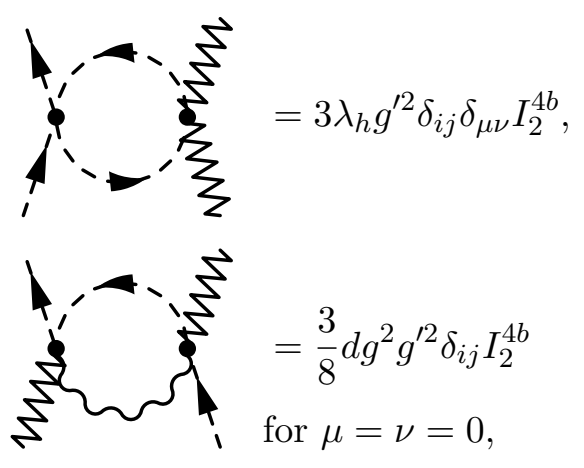

$$
=\frac{9}{8} g^{2} g^{\prime 2} \delta_{i j} \delta_{r s} I_{2}^{4 b}
$$

for $\mu=r, \nu=s$,

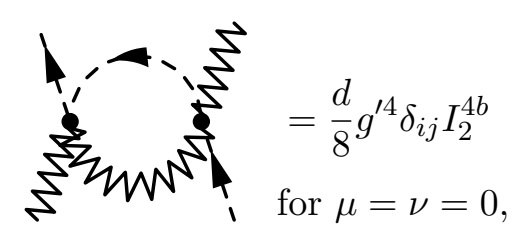

$$
\begin{gathered}
=\frac{3}{8} g^{\prime 4} \delta_{i j} \delta_{r s} I_{2}^{4 b} \\
\text { for } \mu=r, \nu=s,
\end{gathered}
$$
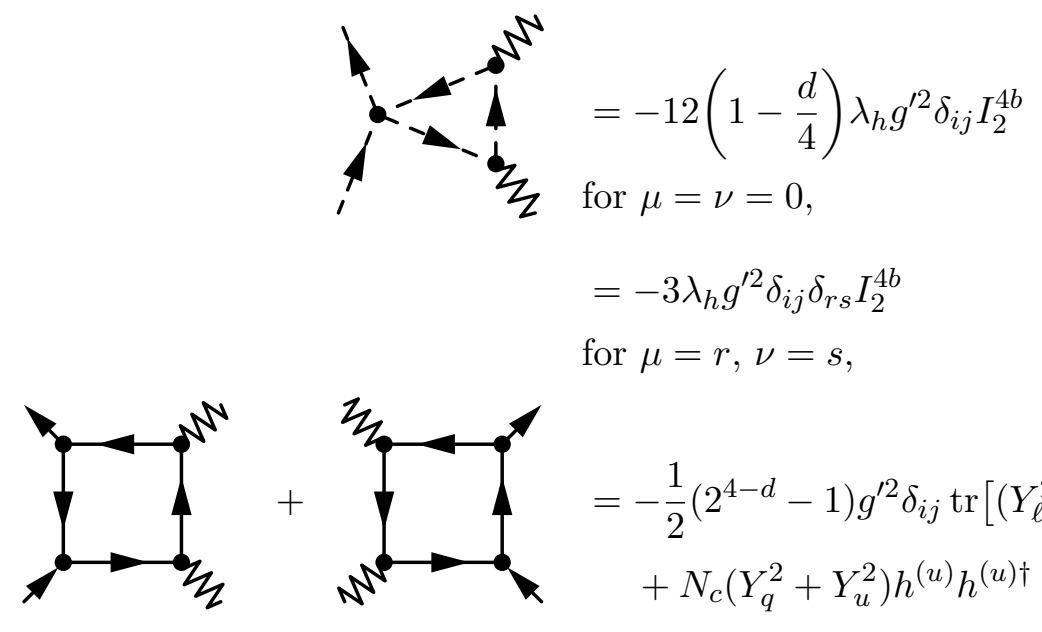

$$
\begin{aligned}
& =-3 \lambda_{h} g^{2} \delta_{i j} \delta_{r s} I_{2}^{4 b} \\
& \text { for } \mu=r, \nu=s,
\end{aligned}
$$

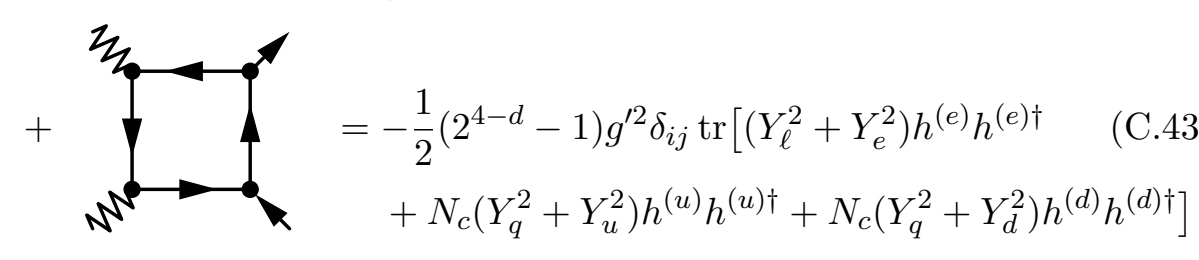




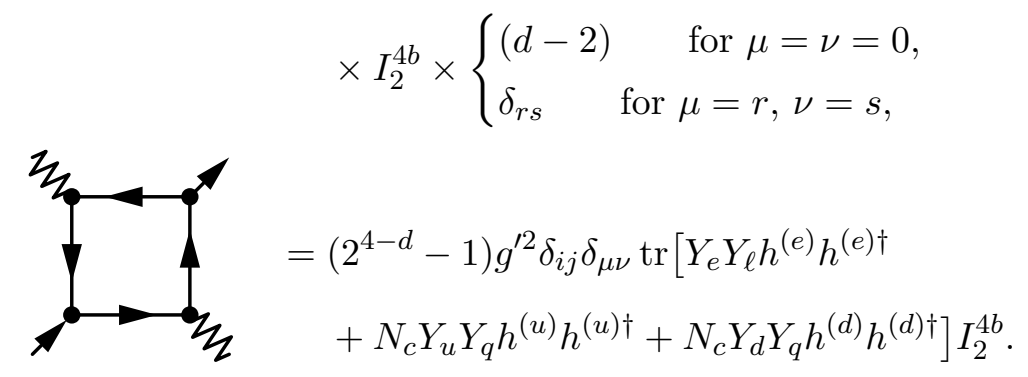

The $\phi^{\dagger i} \phi^{j} A_{0}^{a} B_{0}$ correlator.

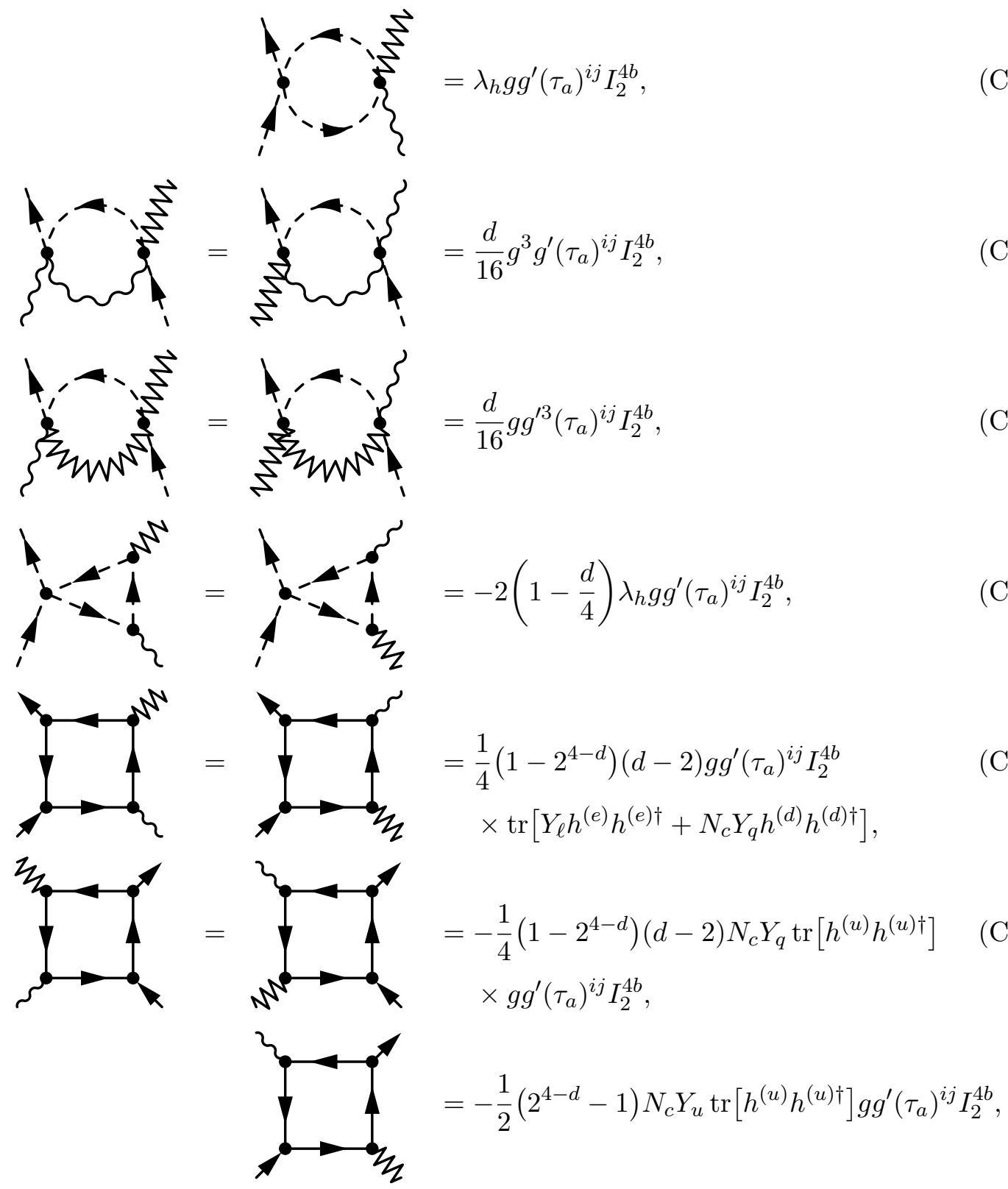




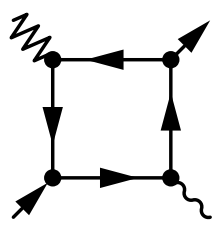

$$
\begin{aligned}
= & \frac{1}{2}\left(2^{4-d}-1\right) \operatorname{tr}\left[Y_{e} h^{(e)} h^{(e) \dagger}+N_{c} Y_{d} h^{(d)} h^{(d) \dagger}\right] \\
& \times g g^{\prime}\left(\tau_{a}\right)^{i j} I_{2}^{4 b} .
\end{aligned}
$$

The $\phi^{\dagger i} \phi^{j} C_{0}^{\alpha} C_{0}^{\beta}$ correlator.
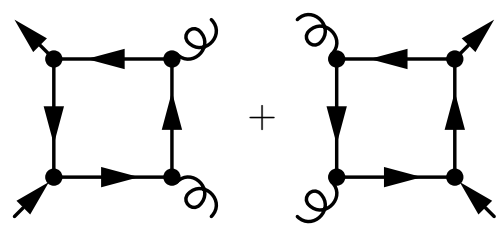

$=2\left(2^{4-d}-1\right)(2-d) g_{s}^{2} \operatorname{tr}\left[h^{(u)} h^{(u) \dagger}+h^{(d)} h^{(d) \dagger}\right] \delta_{i j} \delta_{\alpha \beta} I_{2}^{4 b}$

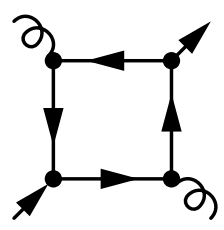

$=2\left(2^{4-d}-1\right) g_{s}^{2} \operatorname{tr}\left[h^{(u)} h^{(u) \dagger}+h^{(d)} h^{(d) \dagger}\right] \delta_{i j} \delta_{\alpha \beta} I_{2}^{4 b}$

Open Access. This article is distributed under the terms of the Creative Commons Attribution License (CC-BY 4.0), which permits any use, distribution and reproduction in any medium, provided the original author(s) and source are credited.

\section{References}

[1] V.A. Kuzmin, V.A. Rubakov and M.E. Shaposhnikov, On the anomalous electroweak baryon number nonconservation in the early universe, Phys. Lett. B 155 (1985) 36 [INSPIRE].

[2] A.G. Cohen, D.B. Kaplan and A.E. Nelson, Progress in electroweak baryogenesis, Ann. Rev. Nucl. Part. Sci. 43 (1993) 27 [hep-ph/9302210] [INSPIRE].

[3] V.A. Rubakov and M.E. Shaposhnikov, Electroweak baryon number nonconservation in the early universe and in high-energy collisions, Usp. Fiz. Nauk 166 (1996) 493 [Phys. Usp. 39 (1996) 461] [hep-ph/9603208] [INSPIRE].

[4] D.E. Morrissey and M.J. Ramsey-Musolf, Electroweak baryogenesis, New J. Phys. 14 (2012) 125003 [arXiv: 1206.2942] [INSPIRE].

[5] M.E. Shaposhnikov, Structure of the high temperature gauge ground state and electroweak production of the baryon asymmetry, Nucl. Phys. B 299 (1988) 797 [INSPIRE].

[6] G.R. Farrar and M.E. Shaposhnikov, Baryon asymmetry of the universe in the minimal Standard Model, Phys. Rev. Lett. 70 (1993) 2833 [Erratum ibid. 71 (1993) 210] [hep-ph/9305274] [INSPIRE].

[7] G.R. Farrar and M.E. Shaposhnikov, Baryon asymmetry of the universe in the standard electroweak theory, Phys. Rev. D 50 (1994) 774 [hep-ph/9305275] [INSPIRE].

[8] M.B. Gavela, P. Hernández, J. Orloff and O. Pene, Standard Model CP-violation and baryon asymmetry, Mod. Phys. Lett. A 9 (1994) 795 [hep-ph/9312215] [INSPIRE]. 
[9] M.B. Gavela, M. Lozano, J. Orloff and O. Pene, Standard Model CP-violation and baryon asymmetry. Part 1: zero temperature, Nucl. Phys. B 430 (1994) 345 [hep-ph/9406288] [INSPIRE].

[10] M.B. Gavela, P. Hernández, J. Orloff, O. Pene and C. Quimbay, Standard Model CP-violation and baryon asymmetry. Part 2: finite temperature, Nucl. Phys. B 430 (1994) 382 [hep-ph/9406289] [INSPIRE].

[11] T. Brauner, O. Taanila, A. Tranberg and A. Vuorinen, Temperature dependence of Standard Model CP-violation, Phys. Rev. Lett. 108 (2012) 041601 [arXiv:1110.6818] [InSPIRE].

[12] T. Brauner, O. Taanila, A. Tranberg and A. Vuorinen, Computing the temperature dependence of effective CP-violation in the Standard Model, JHEP 11 (2012) 076 [arXiv: 1208.5609] [INSPIRE].

[13] K. Kajantie, M. Laine, K. Rummukainen and M.E. Shaposhnikov, Generic rules for high temperature dimensional reduction and their application to the Standard Model, Nucl. Phys. B 458 (1996) 90 [hep-ph/9508379] [INSPIRE].

[14] K. Kajantie, M. Laine, K. Rummukainen and M.E. Shaposhnikov, The electroweak phase transition: a nonperturbative analysis, Nucl. Phys. B 466 (1996) 189 [hep-lat/9510020] [INSPIRE].

[15] K. Kajantie, M. Laine, K. Rummukainen and M.E. Shaposhnikov, Is there a hot electroweak phase transition at $m_{H}$ larger or equal to $m_{W}$ ?, Phys. Rev. Lett. 77 (1996) 2887 [hep-ph/9605288] [INSPIRE].

[16] K. Kajantie, M. Laine, K. Rummukainen and M.E. Shaposhnikov, A nonperturbative analysis of the finite $T$ phase transition in $\mathrm{SU}(2) \times \mathrm{U}(1)$ electroweak theory, Nucl. Phys. B 493 (1997) 413 [hep-lat/9612006] [INSPIRE].

[17] F. Csikor, Z. Fodor and J. Heitger, The strength of the electroweak phase transition at $m_{H} \approx 80 \mathrm{GeV}$, Phys. Lett. B 441 (1998) 354 [hep-lat/9807021] [InSPIRE].

[18] F. Csikor, Z. Fodor and J. Heitger, Endpoint of the hot electroweak phase transition, Phys. Rev. Lett. 82 (1999) 21 [hep-ph/9809291] [INSPIRE].

[19] Y. Aoki, F. Csikor, Z. Fodor and A. Ukawa, The endpoint of the first order phase transition of the $\mathrm{SU}(2)$ gauge Higgs model on a four-dimensional isotropic lattice, Phys. Rev. D 60 (1999) 013001 [hep-lat/9901021] [INSPIRE].

[20] M. Fukugita and T. Yanagida, Baryogenesis without grand unification, Phys. Lett. B 174 (1986) 45 [INSPIRE].

[21] M.A. Luty, Baryogenesis via leptogenesis, Phys. Rev. D 45 (1992) 455 [InSPIRE].

[22] W. Buchmüller, P. Di Bari and M. Plümacher, Leptogenesis for pedestrians, Annals Phys. 315 (2005) 305 [hep-ph/0401240] [inSPIRE].

[23] S. Davidson, E. Nardi and Y. Nir, Leptogenesis, Phys. Rept. 466 (2008) 105 [arXiv: 0802.2962] [INSPIRE].

[24] J. García-Bellido, D. Yu. Grigoriev, A. Kusenko and M.E. Shaposhnikov, Nonequilibrium electroweak baryogenesis from preheating after inflation, Phys. Rev. D 60 (1999) 123504 [hep-ph/9902449] [INSPIRE].

[25] L.M. Krauss and M. Trodden, Baryogenesis below the electroweak scale, Phys. Rev. Lett. 83 (1999) 1502 [hep-ph/9902420] [INSPIRE]. 
[26] E.J. Copeland, D. Lyth, A. Rajantie and M. Trodden, Hybrid inflation and baryogenesis at the TeV scale, Phys. Rev. D 64 (2001) 043506 [hep-ph/0103231] [INSPIRE].

[27] A. Tranberg and J. Smit, Baryon asymmetry from electroweak tachyonic preheating, JHEP 11 (2003) 016 [hep-ph/0310342] [INSPIRE].

[28] Virgo and LigO Scientific collaborations, B.P. Abbott et al., Observation of gravitational waves from a binary black hole merger, Phys. Rev. Lett. 116 (2016) 061102 [arXiv: 1602.03837] [INSPIRE].

[29] C. Grojean and G. Servant, Gravitational waves from phase transitions at the electroweak scale and beyond, Phys. Rev. D 75 (2007) 043507 [hep-ph/0607107] [INSPIRE].

[30] J.M. No, Large gravitational wave background signals in electroweak baryogenesis scenarios, Phys. Rev. D 84 (2011) 124025 [arXiv: 1103.2159] [InSPIRE].

[31] M. Hindmarsh, S.J. Huber, K. Rummukainen and D.J. Weir, Gravitational waves from the sound of a first order phase transition, Phys. Rev. Lett. 112 (2014) 041301 [arXiv: 1304.2433] [INSPIRE].

[32] C. Caprini et al., Science with the space-based interferometer eLISA. II: gravitational waves from cosmological phase transitions, JCAP 04 (2016) 001 [arXiv: 1512.06239] [INSPIRE].

[33] K. Hashino, M. Kakizaki, S. Kanemura, P. Ko and T. Matsui, Gravitational waves and Higgs boson couplings for exploring first order phase transition in the model with a singlet scalar field, Phys. Lett. B 766 (2017) 49 [arXiv:1609.00297] [INSPIRE].

[34] V. Barger, P. Langacker, M. McCaskey, M.J. Ramsey-Musolf and G. Shaughnessy, LHC phenomenology of an extended Standard Model with a real scalar singlet, Phys. Rev. D 77 (2008) 035005 [arXiv:0706.4311] [INSPIRE].

[35] A. Ashoorioon and T. Konstandin, Strong electroweak phase transitions without collider traces, JHEP 07 (2009) 086 [arXiv:0904.0353] [INSPIRE].

[36] T. Robens and T. Stefaniak, Status of the Higgs singlet extension of the Standard Model after LHC run 1, Eur. Phys. J. C 75 (2015) 104 [arXiv:1501.02234] [INSPIRE].

[37] S. Kanemura, M. Kikuchi and K. Yagyu, One-loop corrections to the Higgs self-couplings in the singlet extension, arXiv:1608.01582 [INSPIRE].

[38] S. Kanemura, M. Kikuchi and K. Yagyu, Radiative corrections to the Higgs boson couplings in the model with an additional real singlet scalar field, Nucl. Phys. B 907 (2016) 286 [arXiv: 1511.06211] [INSPIRE].

[39] A. Beniwal, M. Lewicki, J.D. Wells, M. White and A.G. Williams, Gravitational wave, collider and dark matter signals from a scalar singlet electroweak baryogenesis, arXiv: 1702.06124 [INSPIRE].

[40] K. Enqvist, S. Nurmi, T. Tenkanen and K. Tuominen, Standard Model with a real singlet scalar and inflation, JCAP 08 (2014) 035 [arXiv:1407.0659] [INSPIRE].

[41] T. Tenkanen, K. Tuominen and V. Vaskonen, A strong electroweak phase transition from the inflaton field, JCAP 09 (2016) 037 [arXiv: 1606. 06063] [INSPIRE].

[42] S.J. Huber and M.G. Schmidt, Electroweak baryogenesis: concrete in a SUSY model with a gauge singlet, Nucl. Phys. B 606 (2001) 183 [hep-ph/0003122] [INSPIRE]. 
[43] D. O'Connell, M.J. Ramsey-Musolf and M.B. Wise, Minimal extension of the Standard Model scalar sector, Phys. Rev. D 75 (2007) 037701 [hep-ph/0611014] [InSPIRE].

[44] A. Ahriche, What is the criterion for a strong first order electroweak phase transition in singlet models?, Phys. Rev. D 75 (2007) 083522 [hep-ph/0701192] [INSPIRE].

[45] S. Profumo, M.J. Ramsey-Musolf and G. Shaughnessy, Singlet Higgs phenomenology and the electroweak phase transition, JHEP 08 (2007) 010 [arXiv:0705.2425] [INSPIRE].

[46] J.R. Espinosa, T. Konstandin and F. Riva, Strong electroweak phase transitions in the Standard Model with a singlet, Nucl. Phys. B 854 (2012) 592 [arXiv:1107.5441] [INSPIRE].

[47] J.M. Cline and K. Kainulainen, Electroweak baryogenesis and dark matter from a singlet Higgs, JCAP 01 (2013) 012 [arXiv: 1210.4196] [INSPIRE].

[48] P.H. Damgaard, D. O'Connell, T.C. Petersen and A. Tranberg, Constraints on new physics from baryogenesis and Large Hadron Collider data, Phys. Rev. Lett. 111 (2013) 221804 [arXiv: 1305.4362] [INSPIRE].

[49] S. Profumo, M.J. Ramsey-Musolf, C.L. Wainwright and P. Winslow, Singlet-catalyzed electroweak phase transitions and precision Higgs boson studies, Phys. Rev. D 91 (2015) 035018 [arXiv: 1407 .5342] [INSPIRE].

[50] J. Kozaczuk, Bubble expansion and the viability of singlet-driven electroweak baryogenesis, JHEP 10 (2015) 135 [arXiv:1506.04741] [INSPIRE].

[51] P.H. Damgaard, A. Haarr, D. O'Connell and A. Tranberg, Effective field theory and electroweak baryogenesis in the singlet-extended Standard Model, JHEP 02 (2016) 107 [arXiv: 1512.01963] [INSPIRE].

[52] T. Brauner, A. Haarr, T.V.I. Tenkanen, A. Tranberg, A. Vuorinen and D.J. Weir, in preparation.

[53] T. Appelquist and J. Carazzone, Infrared singularities and massive fields, Phys. Rev. D 11 (1975) 2856 [INSPIRE].

[54] E. Braaten and A. Nieto, Effective field theory approach to high temperature thermodynamics, Phys. Rev. D 51 (1995) 6990 [hep-ph/9501375] [INSPIRE].

[55] E. Braaten and A. Nieto, Next-to-leading order Debye mass for the quark-gluon plasma, Phys. Rev. Lett. 73 (1994) 2402 [hep-ph/9408273] [INSPIRE].

[56] J.M. Cline and P.-A. Lemieux, Electroweak phase transition in two Higgs doublet models, Phys. Rev. D 55 (1997) 3873 [hep-ph/9609240] [INSPIRE].

[57] L. Fromme, S.J. Huber and M. Seniuch, Baryogenesis in the two-Higgs doublet model, JHEP 11 (2006) 038 [hep-ph/0605242] [INSPIRE].

[58] J.M. Cline, K. Kainulainen and M. Trott, Electroweak baryogenesis in two Higgs doublet models and B meson anomalies, JHEP 11 (2011) 089 [arXiv:1107.3559] [INSPIRE].

[59] D. Curtin, P. Jaiswal and P. Meade, Excluding electroweak baryogenesis in the MSSM, JHEP 08 (2012) 005 [arXiv: 1203.2932] [INSPIRE].

[60] D.J.H. Chung, A.J. Long and L.-T. Wang, 125 GeV Higgs boson and electroweak phase transition model classes, Phys. Rev. D 87 (2013) 023509 [arXiv:1209.1819] [INSPIRE].

[61] A. Haarr, A. Kvellestad and T.C. Petersen, Disfavouring electroweak baryogenesis and a hidden Higgs in a CP-violating two-Higgs-doublet model, arXiv: 1611.05757 [INSPIRE]. 